\title{
Permanent magnet systems for microfluidic applications
}

\author{
Yannick P. Klein
}
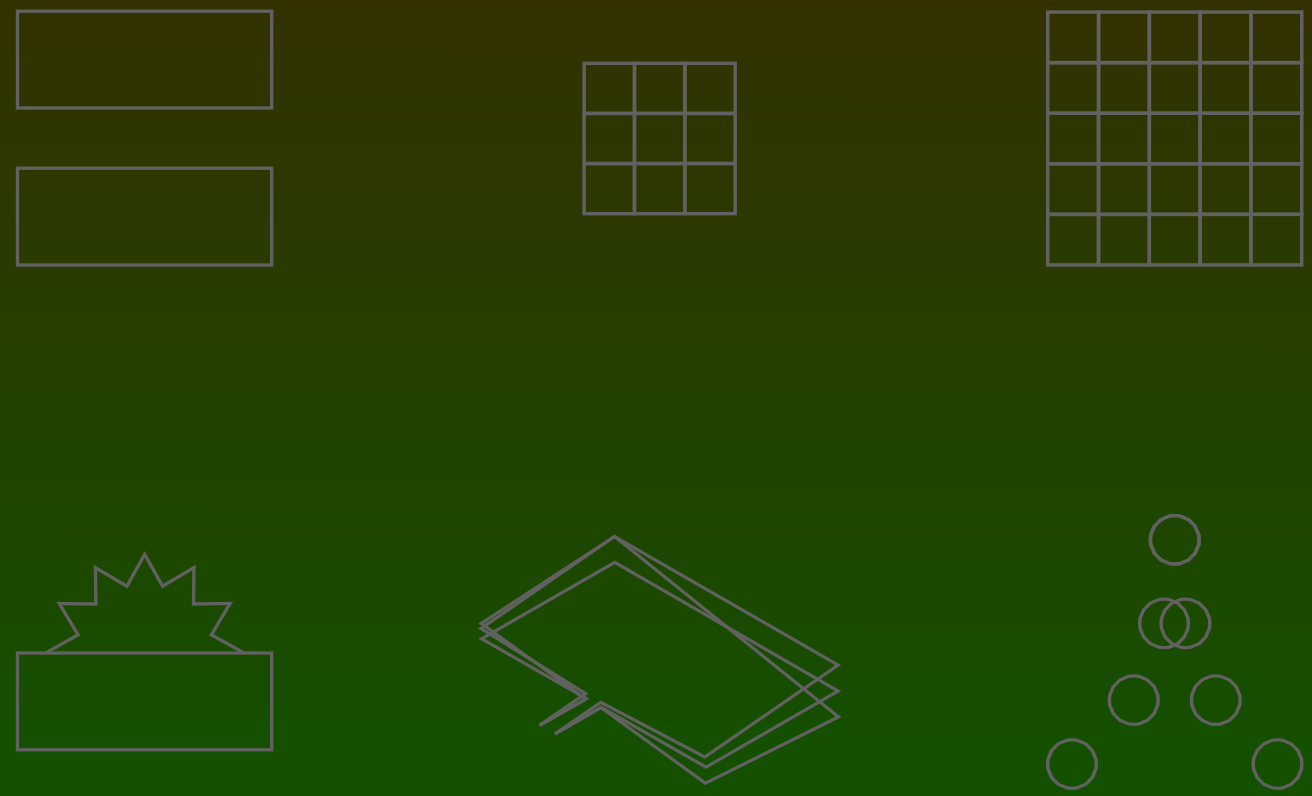


\title{
Permanent MAgnet Systems FOR MICROFLUIDIC APPLICATIONS
}

\author{
DISSERTATION
}

to obtain

the degree of doctor at the University of Twente, on the authority of the rector magnificus, prof.dr.ir. A. Veldkamp,

on account of the decision of the Doctorate Board, to be publicly defended

on Friday, $18^{\text {th }}$ December, 2020 at 12:45

by

\section{Yannick Philippe Klein}

born on 8 May 1990,

in Saarbrücken, Germany 
Graduation committee

prof. dr. J.L. Herek University of Twente (chairman and secretary)

prof. dr. J.G.E. Gardeniers University of Twente (supervisor)

prof. dr.ir. L. Abelmann University of Twente

dr.ir. J. Hogendoorn KROHNE New Technologies B.V.

prof. dr.ir. B. ten Haken University of Twente

prof. dr.ir. J.C. Lötters University of Twente

dr. H. van As

prof. dr. A.G. Webb
Wageningen University \& Research

Leiden University Medical Center

\section{UNIVERSITY OF TWENTE.}

The research described in this dissertation is part of the research programme FLOW+ with project number 15025 , which is (partly) financed by the Dutch Research Council (NWO).

Cover design by Yannick Philippe Klein

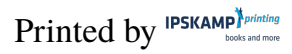

(C) Yannick Philippe Klein, Enschede, The Netherlands, 2020.

Electronic mail address: y.p.klein@utwente.nl

ISBN $978-90-365-5102-1$

DOI 10.3990/1.9789036551021 
This dissertation is approved by prof. dr. J.G.E. Gardeniers University of Twente (supervisor) 
Das Schönste, was wir erleben können, ist das Geheimnisvolle

Albert Einstein 


\section{Contents}

Contents

1 Introduction 1

$1.1 \quad$ History and applications $\ldots \ldots \ldots \ldots \ldots \ldots \ldots \ldots$

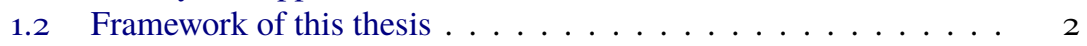

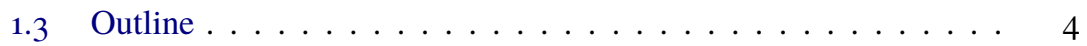

2 Influence of the distribution of the properties of permanent magnets on the field homogeneity of magnet assemblies for mobile NMR 5

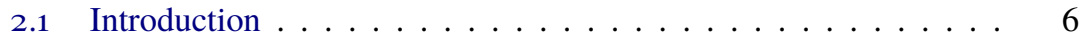

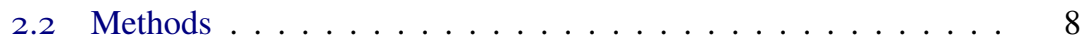

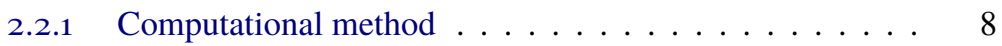

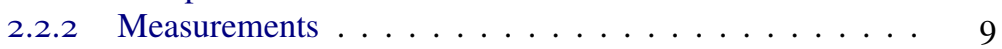

2.3 Results and Discussion . . . . . . . . . . . . . 9

2.3.1 Magnet variation measurements . . . . . . . . . . 9

2.3 .2 Comparison of designs . . . . . . . . . . . 10

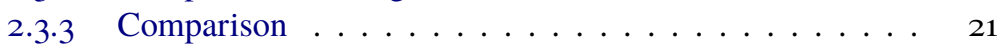

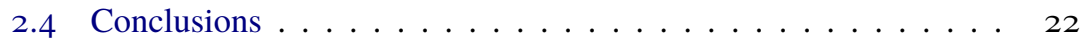

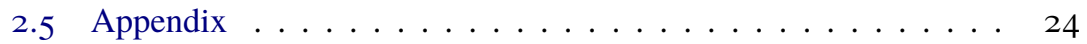

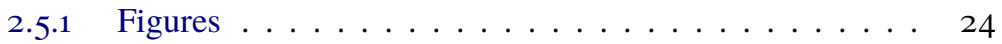

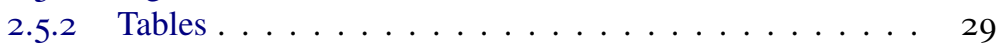

3 Dumbbell Pseudo Halbach Magnet Configuration: Combining high field strength with high homogeneity 33

$3.1 \quad$ Introduction $\ldots \ldots \ldots \ldots \ldots \ldots \ldots \ldots \ldots \ldots \ldots$

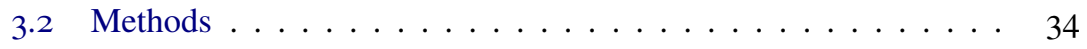

3.2.1 Field calculations $\ldots \ldots \ldots \ldots \ldots \ldots \ldots \ldots$

3.2 .2 Experimental. . . . . . . . . . . . . . . 35

3.3 Results and Discussion . . . . . . . . . . . . . 37

3.3.1 Mechanical shimming: Dumbbell-Halbach $\ldots \ldots . . .37$

3.3 .2 Electric shimming . . . . . . . . . . . . . . . $3^{8}$

3.3.3 Experimental validation $\ldots \ldots \ldots \ldots \ldots \ldots .40$

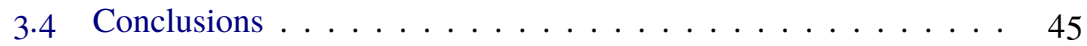

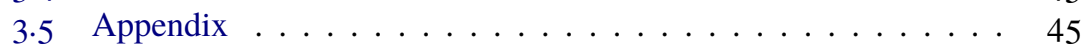


4 Shim on a chip on small scale using ferrofluids 49

4.1 Introduction . . . . . . . . . . . . . . . . . . . 49

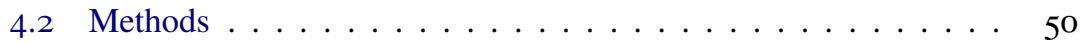

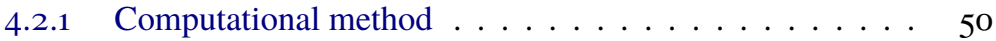

4.2.2 Dumbbell-Halbach ................... 50

4.2 .3 Microfluidic shimming chips ................. 52

4.2.4 VSM-measurements ferrofluid . . . . . . . . . . 53

4.3 Results and Discussion . . . . . . . . . . . . . . 54

4.3.1 Ferrofluidic shimming chips - different saturation magnetization ....................... 54

4.3.2 Ferrofluidic shimming chips - different volumes . . . . . 56

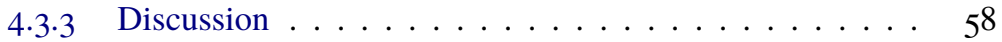

4.4 Conclusions and Outlook ............... 58

5 Magnetic Field Strength Improvement for Lorentz Actuation of a $\mu$-Coriolis Mass Flow Sensor

5.1 Introduction . . . . . . . . . . . . . . 60

5.2 Methods ..................... 60

5.2 .1 Device \& Fabrication . . . . . . . . . . . . . . . 60

5.2 .2 Frequency response . . . . . . . . . . . . 61

5.2.3 Magnetic simulation and assembly .......... 65

$5 \cdot 3$ Results ....................... 66

5.4 Conclusions ...................... 70

6 Trajectory Deflection of Spinning Magnetic Microparticles, the $\begin{array}{ll}\text { Magnus Effect at the Microscale } & 71\end{array}$

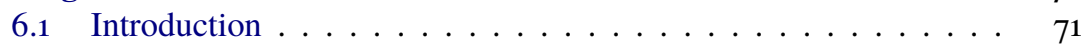

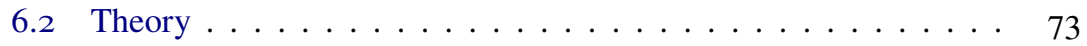

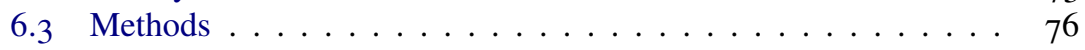

6.4 Results and Discussion . . . . . . . . . . . . . . . 80

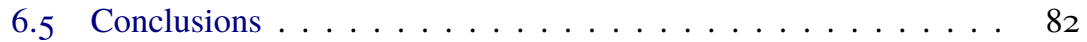

6.6 Appendix ........................ 83

$\begin{array}{llr}7 & \text { Summary } & \mathbf{8 7}\end{array}$

$\begin{array}{lr}\text { Samenvatting } & 89\end{array}$

$\begin{array}{ll}\text { Zusammenfassung } & 91\end{array}$

$\begin{array}{ll}\text { Acknowledgments } & 93\end{array}$

$\begin{array}{ll}\text { Bibliography } & 94\end{array}$ 



\section{Chapter 1}

\section{Introduction}

This chapter gives an overview about the history of permanent magnets and their applications, as well as the aims and outline of this thesis.

\subsection{History and applications}

Permanent magnets are materials, which experience attracting or repelling forces based on their physical properties when exposed to other specific materials. Even though this behavior has been used already 1175 A.C. in the form of a compass by using a so called "Lodestone" on a piece of cork floating on water (Carlson, 1975), it took more than 400 years to understand its link with the earth magnetic-field as the earth has been described as a big magnet by William Gilbert (Lindsay, 1940). John Michell discovered, that the strength of both poles of a magnet are similar, in this early state he published a book where he explained how to fabricate artificial magnets, without using Lodestones (Michell, 1751). In 1820, Hans Christian Oersted discovered the link between electricity and magnetism, a scientific milestone (Oersted, 1820) which was used in 1821, when Faraday placed current-carrying wire in a magnetic field to let it rotate, showing the first time the principle how a modern electric motor works (Faraday, 1821). After a few years of further development, Zenobe Theophile Gramme introduced the fist electric motor and generator of commercial significance in 1871 using carbon steel magnets, fabricated by the rolling process (Gramme, 1871). The development of alnicomagnets (Ni-Co-Al-Fe), first rare-earth magnets ( $\left.\mathrm{SmCo}_{5}, \mathrm{Sm}_{2} \mathrm{Co17}\right)$, NeodyniumIron-Boron ( $\left.\mathrm{Nd}_{2} \mathrm{Fe}_{14} \mathrm{~B}\right)$ magnets in the 2oth century had a major impact on the improvement of permanent magnet-involving applications (Livingston, 1990). Whether they are present in communication- and entertainment electronics, cars, wind turbines or other industrial equipment in e.g. optical drives, hard discs, loudspeakers, electric motors, generators, sorting machines or lifting tools, magnets became indispensable in the modern world (Coey, 2002).

One specific application of permanent magnets is the NMR-spectroscopy, which requires high and homogeneous magnetic fields, for instance to analyse the chemical 
composition of liquids (see e.g. (Levitt, 2008)). The current trends in NMR instrument development are two-fold: one trend is to ever more powerful highend systems with spectral resolution below $0.1 \mathrm{ppb}$ (Hashi et al., 2015), using He-cooled superconducting magnets operating at $1 \mathrm{GHz}{ }^{1} \mathrm{H}$ resonance, $\sim 5 \mathrm{~m}$ height, $\sim 15 \mathrm{t}$ weight, and $\sim 12 \mathrm{M} €$ cost. On the other side of the spectrum are the more affordable $(\sim 25 \mathrm{k} €)$ bench-top systems for chemical labs (Nanalysis, 2020.11.04; PicoSpin8o, 2020.11.04; Pulsar, 2020.11.04; Spinsolve, 2020.11.04), with permanent magnets giving $40 \mathrm{MHz}-100 \mathrm{MHz}{ }^{1} \mathrm{H}$ resonance, $<60 \mathrm{~cm}$ height, $<150 \mathrm{~kg}$ weight, and resolution $\sim 20 \mathrm{ppb}$. Specialized robust process-analytical systems for production environments (Aspect, 2020.11.04; J.C. Edwards, 2010) $(\sim 60 \mathrm{MHz}, 1.8 \mathrm{~m} \max , 600 \mathrm{~kg} \max )$ can also be ranked in the latter category. Most systems employ standard $5 \mathrm{~mm}$ OD tubes with $>0.3 \mathrm{~mL}$ liquid sample, the process systems which work online with reactors typically in $3 \mathrm{~mm}-10 \mathrm{~mm}$ OD flow pipes and $2 \mathrm{ml}-5 \mathrm{ml}$ sample volume. It should be noted that the static magnetic field in these systems is commonly expressed as the Larmor frequency of the nuclear spin of the proton, ${ }^{1} \mathrm{H}$, in MHz. For example, a $\sim 1 \mathrm{~T}$ magnet, as will be discussed in this thesis, corresponds to $42.6 \mathrm{MHz}$. The magnet uniformity is conveniently expressed in ppm ( $\mu$ Tesla/Tesla), which directly relates to the spectral resolution of NMR, expressed in $\mathrm{Hz}$ or more commonly in $\mathrm{ppm}(\mathrm{Hz}$ per $\mathrm{MHz})$. In this thesis we will use ppm to indicate magnetic field uniformity (over a defined volume). To keep the NMR systems light and mobile, one can work with a system of permanent magnets, which can nowadays be manufactured in any size and shape, although only with a limited magnetic field strength. The main challenges in the design of such systems is to produce a high field and to strongly reduce field inhomogeneities, which are initially caused by chosen magnet-design and the properties of the magnetic material. Another challenge is to obtain enough NMR sensitivity because intrinsically the magnetic effects caused by the nuclear spins are very small, in particular for the atoms that are of interest in chemical applications, such as Hydrogen and Carbon (Zalesskiy et al., 2014). A special magnet configuration has been invented by Klaus Halbach in 1980, which reduces the stray field while enhancing the field at the ring-center. This configuration is frequently used in modern NMR-applications and a key component of this thesis (Halbach, 1980).

\subsection{Framework of this thesis}

The aim of this thesis is to characterize and design permanent magnet configurations for different microfluidic applications. The research was part of the research programme FLOW+ with project number 15025 , which is (partly) financed by the Dutch Research Council (NWO), Bronkhorst High-Tech B.V. and KROHNE New Technologies B.V.. The project, entitled "Microflow Magnetic Resonance", is in close collaboration with Electronic Instrumentation Laboratory, led by professor Makinwa, at the Technical University of Delft, where the Ph.D. student Eren Aydin has worked on the excitation and detection electronics for magnetic resonance 
systems. The key element in a miniaturized system that applies magnetic resonance for chemical analysis (i.e. NMR) or for measuring the flow rate of single or multiphase fluids, is a magnet system that achieves a static magnetic field $\mathrm{B}_{0}$ that is very homogeneous over the complete sample volume (to be called the "region of interest") and that remains stable in a realistic operating environment. The target that was set for this project, namely a system with a size in the range of centimeters and with minimized power consumption, dictates that permanent magnets should be applied and that preferably passive elements should be used to optimize (to "shim") the field homogeneity in the region of interest. A further requirement is a flow-through system, with a sample flow tube with an ID between $0.1 \mathrm{~mm}$ and $3 \mathrm{~mm}$ with a typical effective measurement volume of the order of $1 \mu \mathrm{L}$ and liquid flow rates in the $\mu \mathrm{L} / \mathrm{min}$ range. For that reason the region of interest was set as a sample length of a few $\mathrm{mm}$ for a flow tube with a diameter below $1 \mathrm{~mm}$, flow aspects were not studied in this thesis. A special magnet configuration has been invented by Klaus Halbach in 1980 , which reduces the stray field while enhancing the field at the ring-center (Halbach, 1980). The cylindrical Halbach array is the most studied permanent magnet design for miniaturized NMR (Zalesskiy et al., 2014), and will be a key component of this thesis. A Halbach design gives the desired $\mathrm{B}_{0}$ homogeneity for relatively strong (up to ca. $1 \mathrm{~T}$ ) field inside the hollow core of the cylinder, without a significant stray field outside the magnet. The latter is important for applications in an environment with steel components (such as reactors and piping), or near delicate electronics. Halbach magnets are commercially available with high quality and are reasonably priced. Magnets made from the more common $\mathrm{NdFeB}$ material $\left(\mathrm{T}_{\max } 50^{\circ} \mathrm{C}\right)$ or from the high-temperature material $\mathrm{SmCo}_{5}\left(\mathrm{~T}_{\max } 300^{\circ} \mathrm{C}\right)$ are available from different suppliers. Since the Halbach configuration has been reported suitable for portable magnetic resonance systems (Ha et al., 2014; Zalesskiy et al., 2014) and has been the method of choice in Krohne's large multiphase flow meters, this will be the basis for our device development. The study in this thesis has made extensive use of electromagnetic modeling to arrive at the best Halbach configuration for the desired scale of tubing, with a modeling focus on general design rules and shimming methods. The latter is considered important for future product developments, where different volumetric requirements may hold. The size of the magnet for this project will be in the range $0.5 \mathrm{~T}-1 \mathrm{~T}$ (ca. $20 \mathrm{MHz}$ to $40 \mathrm{MHz}{ }^{1} \mathrm{H}$ resonance), which overlaps the field application ranges of quality control (using relaxometry) and process monitoring (spectroscopy) (Mitchell et al., 2014). The target $\mathrm{B}_{0}$ field uniformity was set at $0.1 \mathrm{ppm}$, which cannot be reached by the Halbach alone, but needs shimming elements such as small electronic coils to further homogenize the field at the measurement location. Furthermore, thermal regulation of the $\mathrm{B}_{0}$ field is particularly relevant for long-term measurement schemes. This was not studied in detail in this thesis, because it was the topic of the Ph.D. student at TU Delft, but it was taken into consideration during Hall probe measurements, which were performed in a controlled temperature environment. Details are given in the following chapters. 


\subsection{Outline}

In chapter 2 we present the optimization procedure of planar and Halbach permanent magnet configurations. We measured several magnet properties and studied their influence on the field-homogeneity based on a Monte-Carlo simulation. In chapter $\mathbf{3}$ we designed a new magnet configuration for a mobile NMR-device in combination with planar electric shimming chips. Chapter 4 reviews the possibility of using ferrofluids in a microfluidic chip to improve the field-homogeneity of the previous mentioned magnet configuration. Chapter 5 compares three different magnet configurations for Lorentz actuation of a $\mu$-Coriolis mass flow sensor in order to improve its signal amplitude. Chapter 6 shows a new method to sort magnetic particles on small scale, based on the Magnus force. Chapter 7 is a summary of the research which has been done in this thesis. 


\title{
Chapter 2
}

\section{Influence of the distribution of the properties of permanent magnets on the field homogeneity of magnet assemblies for mobile NMR}

\begin{abstract}
We optimized the magnetic field homogeneity of two canonical designs for mobile microfluidic NMR applications: two parallel magnets with an air gap and a modified Halbach array. Along with the influence of the sample length, general design guidelines will be presented. For a fair comparison the sensitive length of the sample has been chosen to be the same as the gap size between the magnets to ensure enough space for the transmitting and receiving unit, as well as basic electric shimming components. Keeping the compactness of the final device in mind, a box with an edge length 5 times the gap size has been defined, in which the complete magnet configuration should fit. With the chosen boundary conditions, the simple parallel cuboid configuration reaches the best homogeneity without active shimming, while the PseudoHalbach configuration has the highest field strength, assuming perfect magnets. However, permanent magnet configurations suffer from imperfections, which results in worse magnetic field homogeneities than expected from simulations using a fixed optimized parameter set. We present a sensitivity analysis for a magnetic cube and the results of studies of the variations in the magnetization and angle of magnetization of magnets purchased from different suppliers, composed of different materials and coatings, and of different sizes. We performed a detailed Monte Carlo simulation on the effect of the measured distribution of magnetic properties on the mentioned configurations.
\end{abstract}

This chapter is based on "Y.P.Klein, L. Abelmann, J.G.E.Gardeniers, Influence of the distribution in properties of permanent magnets on the field homogeneity of magnet assemblies for mobile NMR" under review for IEEE Transactions on Magnetics 


\section{Chapter 2 - Influence of the distribution of the properties of permanent magnets 6 \\ on the field homogeneity of magnet assemblies for mobile NMR}

\subsection{Introduction}

Low-field and low-cost mobile microfluidic nuclear magnetic resonance (NMR) sensors are very suitable for applications in chemical process industry and in research, for example chemical analysis, biomedical applications, and flow measurements (Danieli et al., 2010; Kreyenschulte et al., 2015; Lee et al., 2008; Meribout and Sonowan, 2019; Mitchell et al., 2014; Mozzhukhin et al., 2018; Sørensen et al., 2014, 2015; Zalesskiy et al., 2014). The design of a permanent magnet for an NMR sensor requires both a strong magnetic field and a high field homogeneity within a defined region of interest. In NMR, a high external magnetic field results in a high spectral resolution and detection sensitivity.

However, field-inhomogeneities compromise the spectral resolution. Our aim with this research was to determine how the distribution of the properties of permanent magnets affect the magnetic field homogeneity of magnet configurations for mobile NMR devices.

In the literature, several magnet shapes for mobile NMR sensors have been reported. A broad overview of magnet developments up to 2009 can be found in Demas et al. (Demas and Prado, 2009). U-shaped single-sided magnets (Blümich et al., 1998; Meethan et al., 2014) and magnets with specially shaped iron pole magnets (Marble et al., 2005) have been used to explore surfaces, mobile PseudoHalbach configurations (Vogel et al., 2016) and two cylindrical magnets (Sun et al., 2013) have been applied for solid and liquid NMR measurements. While the Pseudo-Halbach generates a higher field, ranging from 0.7 to $2.0 \mathrm{~T}$ (Danieli et al., 2010; Moresi and Magin, 2003; Tayler and Sakellariou, 2017) compared to 0.35 to $0.6 \mathrm{~T}$ for the other configurations (Blümich et al., 1998; Lee et al., 2008; Marble et al., 2005; Meethan et al., 2014), the reported field homogeneities without electric shimming seem to be independent of the design, ranging from $20 \mathrm{ppm}$ to $606 \mathrm{ppm}$ (Chen and $\mathrm{Xu}$, 2007; Lee et al., 2008; Meribout and Sonowan, 2019; Moresi and Magin, 2003; Sahebjavaher et al., 2010; Sun et al., 2013). Comparing the two most reported mobile liquid NMR sensors, it further stands out that there is no obvious relation between the size of the sensor and the choice of the magnet configuration.

To achieve more insight into possible guidelines for the magnet design, in this paper a modeling study will be presented from which the homogeneity and field strength at specific locations in the gap of the magnet configuration is derived numerically. It is widely experienced that after building such a permanent magnet configuration, the homogeneity reached in practice does not exhibit the same results as in the simulation (Ambrisi et al., 2010; Danieli et al., 2010; Horton et al., 1996; Moresi and Magin, 2003; Soltner and Blümler, 2010), which can be caused by several factors. The magnetization of permanent magnets depends highly on the temperature, as well as on the remanent magnetization (Haavisto et al., 2011). This remanent magnetization can change over time due to shock-induced demagnetization ( $\mathrm{Li}$ et al., 2013; Royce, 1966), external magnetic fields (Lee et al., 2011), a degrading of the magnetic material caused by oxidation (Li et al., 2003), as well as broken or chipped off pieces (since magnets are very brittle) (Horton 

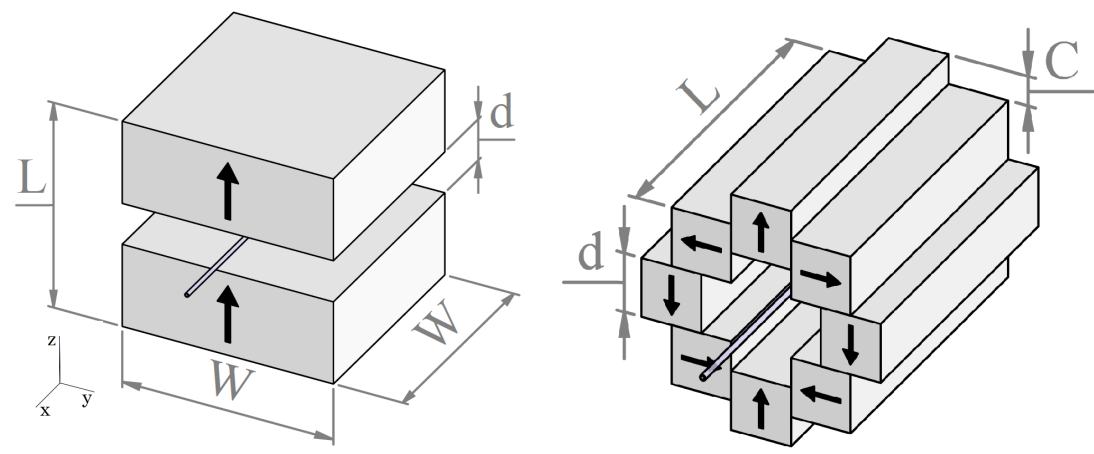

FIGURE 2.1 - Left: Schematic view of the cuboid configuration. Right: Schematic view of the Pseudo-Halbach configuration. The arrows indicate the magnetization of each individual magnet. The sample tube is indicated between the magnet.

et al., 1996). Next to material related differences, fabrication inaccuracies such as variations in the dimensions and magnetization angles affect the field created by a permanent magnet. On top of that, magnet configurations can never be assembled perfectly. Errors in placement may induce a tilt or an axial offset of the magnet. We carried out an extensive numerical sensitivity analysis of a single cubic magnet using these variations. We measured the variations in the magnetization and magnetization angle of magnets composed of different materials, with different coatings, and with different sizes, obtained from different manufacturers. The two main magnet configurations investigated are a system of two parallel magnets and a Pseudo-Halbach configuration (Demas and Prado, 2009), shown in Fig. 2.1. One configuration of each type has been designed and optimized for the following boundary conditions. The region of interest within the channel $(s)$ has been chosen to be the same as the gap size $(d)$. For example: In case a maximal magnet size of $50 \mathrm{~mm} \times 50 \mathrm{~mm} \times 50 \mathrm{~mm}$ is required, the gap size turns out to be $10 \mathrm{~mm}$. All dimension specifications are scalable and will be normalized by the gap length. Scaling the dimensions bigger or smaller will result in an increased or decreased sample lenth relative to the dimensions of the gap, while the magnetic field properties within the region of interest will stay the same. The magnetic field has been normalized to the saturation magnetization of the used magnetic material. The cuboid configuration consists of two cuboid magnets with a height of $2 d$ and a width of $4.72 d$. The Pseudo-Halbach configuration consists of eight bar magnets, each with the dimensions $d \times d \times 5 d$. The measured variations in the magnets have been used to perform a Monte Carlo simulation to provide insight into how the homogeneity of those configurations varies after assembling. The results have been verified with field measurements done with a Tesla meter. The sample channel in most published microfluidic NMR sensors has a high ratio of sample length over inner diameter $\left(s / d_{\mathrm{i}}\right)$ (5.0 over $0.4 \mathrm{~mm}$ in (Gardeniers et al., 2009), 30 over $1.0 \mathrm{~mm}$ in (Kalfe et al., 2015), and 2.9 over $0.15 \mathrm{~mm}$ in (McDonnell 
Chapter 2 - Influence of the distribution of the properties of permanent magnets 8 on the field homogeneity of magnet assemblies for mobile NMR TABLE 2.1 - Purchased permanent magnets.

\begin{tabular}{|c|c|c|c|c|}
\hline Manufacturer & $\begin{array}{l}\text { Dimension } \\
{[\mathrm{mm}]}\end{array}$ & Material & Coating & Abbreviation \\
\hline Supermagnete & $45^{\times} 30^{*}$ & $\begin{array}{l}\mathrm{NdFeB} \\
(\mathrm{N} 45)\end{array}$ & $\mathrm{Ni}-\mathrm{Cu}-\mathrm{Ni}$ & $\mathrm{Su} 45^{\mathrm{Nd}} 45^{\mathrm{NCN}}$ \\
\hline Supermagnete & $7 \times 7 \times 7$ & $\begin{array}{l}\mathrm{NdFeB} \\
\left(\mathrm{N}_{42}\right)\end{array}$ & $\mathrm{Ni}-\mathrm{Cu}-\mathrm{Ni}$ & $\mathrm{Su}_{7} \mathrm{Nd} 42 \mathrm{NCN}$ \\
\hline Supermagnete & $7 \times 7 \times 7$ & $\begin{array}{l}\mathrm{NdFeB} \\
\left(\mathrm{N}_{42}\right)\end{array}$ & $\mathrm{Ni}-\mathrm{Cu}$ & $\mathrm{Su}_{7} \mathrm{Nd} 42 \mathrm{NC}$ \\
\hline HKCM & $7 \times 7 \times 7$ & $\begin{array}{l}\mathrm{NdFeB} \\
\left(\mathrm{N}_{35}\right)\end{array}$ & $\mathrm{Ni}$ & $\mathrm{HK}_{7} \mathrm{Nd}_{35^{\mathrm{N}}}$ \\
\hline HKCM & $7 \times 7 \times 7$ & $\begin{array}{l}\mathrm{Sm}_{2} \mathrm{Co} 17 \\
(\mathrm{YXG} 28)\end{array}$ & $\mathrm{Ni}$ & $\mathrm{HK}_{7} \mathrm{Sm} 28 \mathrm{~N}$ \\
\hline $\begin{array}{l}\text { Schallenkammer } \\
\text { Magnetsysteme }\end{array}$ & $7 \times 7 \times 7$ & $\begin{array}{l}\mathrm{Sm}_{2} \mathrm{Co} 17 \\
(\mathrm{YXG} 26 \mathrm{H})\end{array}$ & - & $\mathrm{Sc}_{7} \mathrm{Sm} 26$ \\
\hline
\end{tabular}

*diameter $\times$ height

et al., 2005)). Therefore we focus on a high field homogeneity in mainly one dimension.

\subsection{Methods}

\subsubsection{Computational method}

The simulations have been done using CADES simulation software, completely described by Delinchant et al. (Delinchant et al., 2007). The magnetic interactions are modeled with the MacMMems tool, which uses the Coulombian equivalent charge method to generate a semi-analytic model.

$$
\begin{array}{r}
\mathbf{H}(\mathbf{r})=\iint_{S} \frac{\sigma_{S} \cdot\left(\mathbf{r}-\mathbf{r}^{\prime}\right)}{\left|\mathbf{r}-\mathbf{r}^{\prime}\right|^{3}} d s+\iiint_{V} \frac{\rho_{V} \cdot\left(\mathbf{r}-\mathbf{r}^{\prime}\right)}{\left|\mathbf{r}-\mathbf{r}^{\prime}\right|^{3}} d v \\
\sigma_{S}=\mathbf{M} \cdot \mathbf{n}, \rho_{V}=-\operatorname{div}(\mathbf{M})
\end{array}
$$

Here, $\mathbf{H}$ is the magnetic field intensity and $\mathbf{M}$ the magnetisation of the permanent magnet (both in A/m), $\mathbf{r}$ and $\mathbf{r}^{\prime}$ define the observation point and its distance to the elementary field source enclosed by $d s$ and $d \nu, \sigma_{S}$ and $\rho_{V}$ are the surface and volume charge, and $\mathbf{n}$ the unit vector normal to the surface.

The CADES framework, including a component generator, component calculator, and component optimizer, generated the final equations, which are used to calculate and optimize the designs. 
TABLE 2.2 - Measured variations in magnetization and magnetization angle of magnets with different materials, coatings, sizes and manufacturers.

\begin{tabular}{lcc}
\hline \hline Magnet & $\frac{B_{\text {std }}}{B_{\text {mean }}}[\%]$ & $\phi\left[^{\circ}\right]$ \\
\hline Su45Nd45NCN & $0.7(3)$ & $0.0(1)$ \\
Su7Nd42NCN & $0.8(2)$ & $0.7(2)$ \\
Su7Nd42NC & $0.6(3)$ & $0.0(1)$ \\
HK 7Nd $35 N$ & $0.3(3)$ & $0.4(2)$ \\
HK 7 Sm28N & $1.0(3)$ & $0.2(1)$ \\
Sc7Sm26 & $1.6(2)$ & $1.0(2)$ \\
\hline \hline
\end{tabular}

\subsubsection{Measurements}

The variations in the properties of the magnets have been measured with a $3 \mathrm{D}$ Hall-probe (THM1176 Three-axis Hall Magnetometer, Metrolab). The setup for the configuration measurements contains a stable temperature environment $\left( \pm 0.5^{\circ} \mathrm{C}\right)$ and a Hall sensor from Projekt Elektronik GmbH (Teslameter 3002/Transverse Probe T3-1,4-5,0-70) in combination with a motorized linear stage. Since the sensor is in a fixed position and only the magnet was moved for the measurement, field variations within the oven have no influence on the measurement. Different kinds of magnets have been purchased. We chose different materials, coatings, sizes and manufacturers, shown in Table 2.1.

\subsection{Results and Discussion}

\subsubsection{Magnet variation measurements}

We have measured the variations in the magnetization and magnetization angle of magnets obtained from different companies (Supermagnete, HKCM and Schallenkammer Magnetsysteme), compositions (NdFeB N45, NdFeB N42, Sm2Co17 $\mathrm{YXG}_{28}$, Sm2Co17 YXG26H), coatings (Ni-Cu-Ni, Ni-Cu, Ni, no coating), and sizes (cylinders with a diameter of $45 \mathrm{~mm}$ and height of $30 \mathrm{~mm}$ or cubes of $7 \mathrm{~mm} \times 7 \mathrm{~mm} \times 7 \mathrm{~mm}$ ). An overview is given in Table $2.2 *$.

On average, the magnetization varies by $1 \%$ of $B_{\text {mean }}$ (Fig. 2.2). The cylindrical magnet, which has a more than 50 times higher magnetic volume than the cubes, shows roughly the same variation in magnetization. From this, we can conclude that inaccuracies in the dimensions are not the main cause of the variation in the magnetization. The uncoated $\mathrm{Sm}_{2} \mathrm{Co} 17$ shows a higher variation in magnetization than the coated magnets, which could be caused by oxidation or small damage to the magnet since unprotected sharp edges of magnets tend to break off easily. Different coatings do not show a clear trend regarding the magnetization standard variation or the variation in the magnetization angle. The offset angle varies on

\footnotetext{
*The values between brackets are the absolute standard errors of the last shown digits.
} 


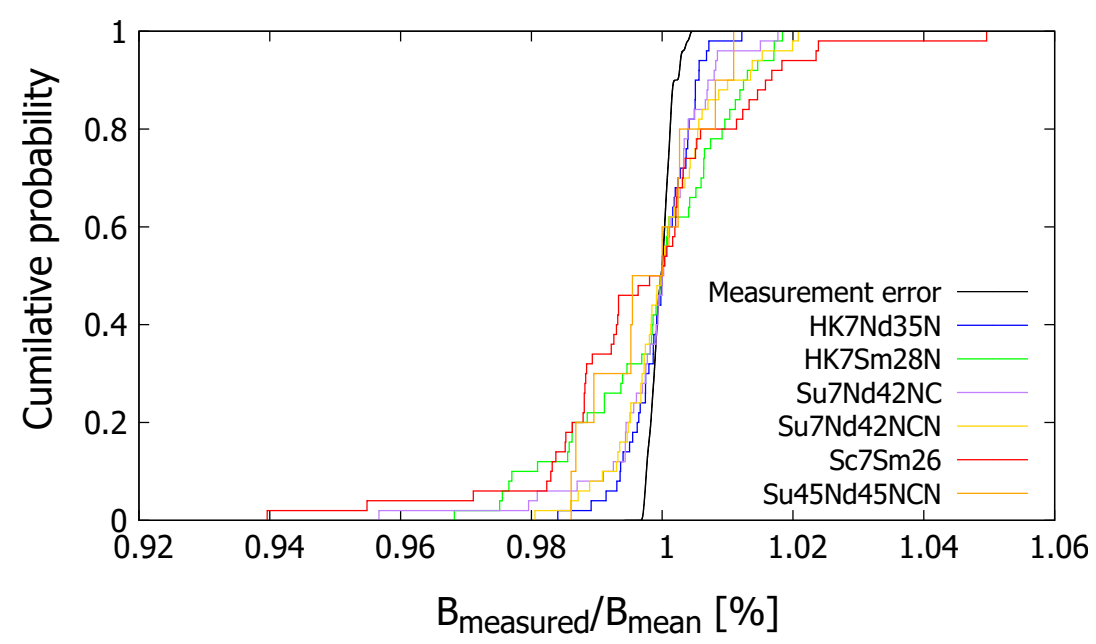

FIGURE 2.2 - Measured cumulative distribution of the field direction for a range of commercial magnets. The sensitivity limit has been obtained from measuring 50 times the same magnet, indicated by the black line. We measured 10 different magnets with a diameter of $d=45 \mathrm{~mm}$ and a height of $h=30 \mathrm{~mm}$ (orange), and 50 magnets with a size of $7 \mathrm{~mm} \times 7 \mathrm{~mm} \times 7 \mathrm{~mm}$ each of the other kinds of material or manufacturer. On average, commercial magnets have a magnetization variation of less than $1 \%$.

average by less than $1^{\circ}$ (Fig. 2.3). There is no clear relation between the variation in magnetization strength or orientation and material, coating or manufacturer.

\subsubsection{Comparison of designs}

\section{Optimization procedure}

To optimize the magnet configurations, the field inhomogeneity of its $z$-component was defined as the root mean square of the difference between the mean field $B_{\text {mean }}$ and the field along the sample $B_{0}$ with the sample size (length of interest) $s$ related to the mean field:

$$
\frac{\frac{1}{s} \int_{0}^{s} \sqrt{\left(B_{0}-B_{\text {mean }}\right)^{2}} d x}{B_{\text {mean }}}
$$

For a better understanding of the different magnet configurations, we start with a description of the cuboid case. The cuboid configuration can be found in Fig. 2.1. Fig. 2.4 shows the magnetic field along the sample of the optimized 


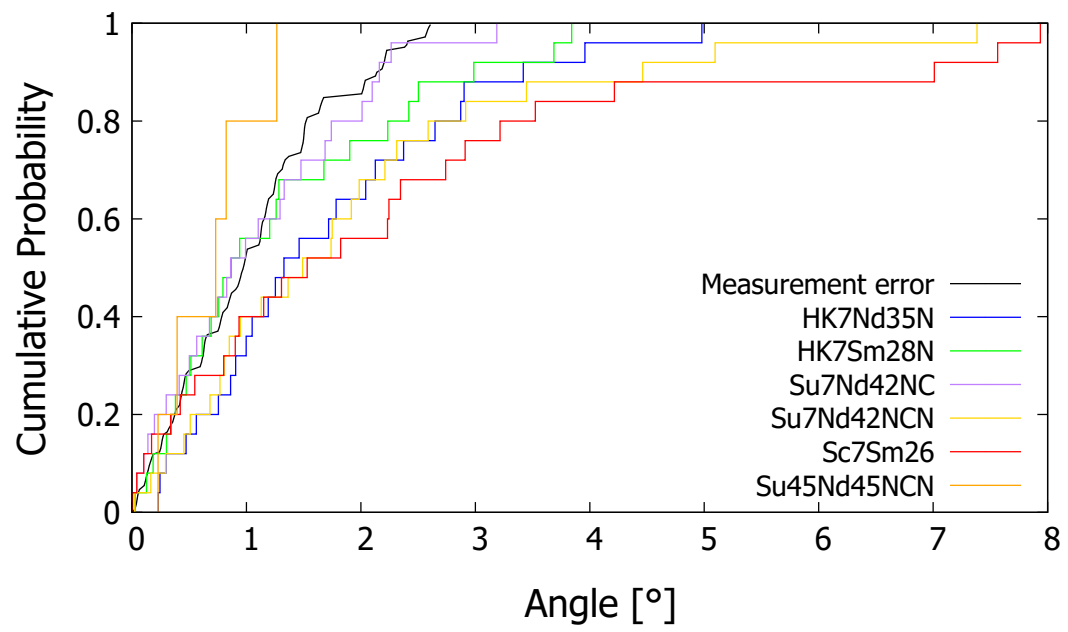

FIGURE 2.3-Measured cumulative distribution of the field direction for a range of commercial magnets. On average, commercial magnets have a field direction variation of less than $1^{\circ}$. The line indicated 'Measurement error' shows a standard deviation of $0.7^{\circ}$.

cuboid configuration, in which the field is the same in the center and at the edge of a sample. The field is symmetric, showing a valley in the middle and two peaks in the directions of the edges. After those maxima, the field decreases with the distance to the center.

Fig. 2.5 shows how the field homogeneity develops with increasing sample size (length of interest) while keeping the previously optimized parameter set constant. Three regions can be seen. In the first one the field increases from $0.50035 B_{\mathrm{S}}$ to $0.50041 B_{\mathrm{s}}$, which means that the minimum field of $0.50035 B_{\mathrm{s}}$ stays the same while the maximum field is increasing until it reaches its global maximum, hence the inhomogeneity is also increasing. In the second region the inhomogeneity stays almost constant. In the third region the field decreases below the previous minimum, which results in a drastic increase of the inhomogeneity. Because of this, the lowest inhomogeneity between two points can be either reached keeping the sample as short as possible or when the field difference at the sample edges and the middle are the same. This information is important for the following optimization processes.

Cuboid The cuboid configuration (Fig. 2.1) consists of two parallel cuboid magnets. The length $L$ of the whole configuration has been chosen to be five times the gap size $d$. The width $W$ was used to tune the field in between the magnets. 
Chapter 2 - Influence of the distribution of the properties of permanent magnets

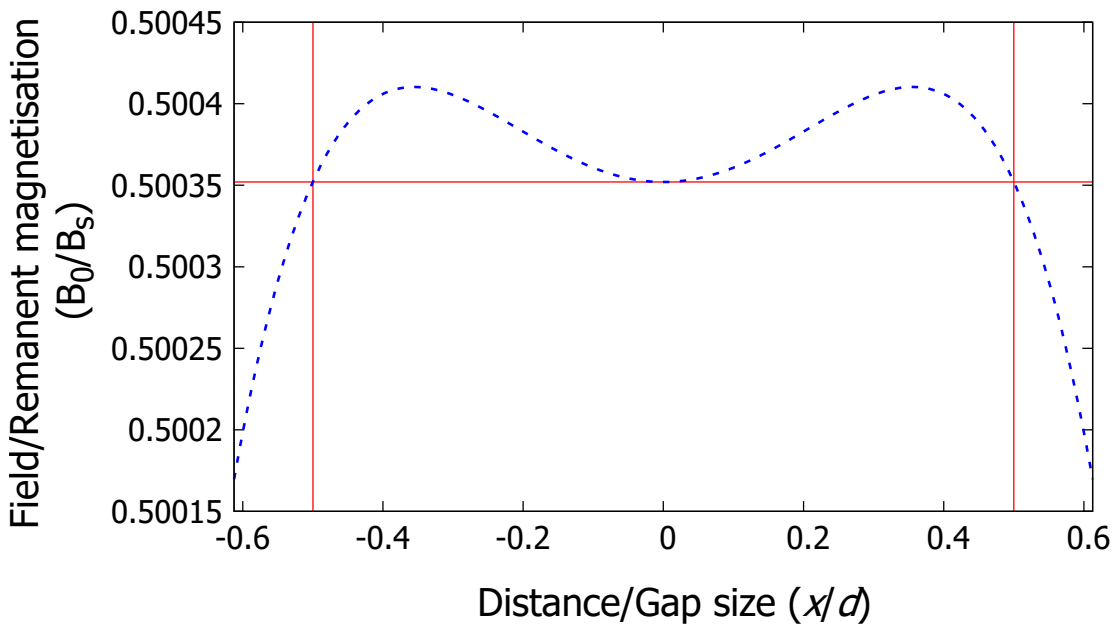

FIGURE 2.4 - Field profile of the cuboid configuration as a function of the relative distance from the center between the magnets. In the optimized situation for a sample size equal to the gap size, the field in the center equals the field at the edge $(x=0.5 d)$.

This subsection will show how to optimize a cuboid configuration using its width to tune the homogeneity of the field. From the previous section we know that we have to find a width for which the field in the center and at the sample edge is the same.

Fig. 2.6 shows that the magnetic field in the center increases to its maximum of $0.54 B_{\mathrm{S}}$ at a width of $\pm 3.0375 \mathrm{~d}$. Increasing the width further results in a reduction of the magnetic field, caused by the larger distance from the edges of the magnet to the center. The difference between the magnetic field in the center and at the sample edge increases until it reaches a maximum, when the width equals the gap size. From this point the difference decreases until it reaches a minimum at a width/gap ratio of 4.72 . The stray field at a distance equal to the gap size is $0.24 B_{\mathrm{s}}$.

Pseudo-Halbach The Pseudo-Halbach configuration (Fig. 2.1) consists of eight magnets, arranged in such a way that the field in the bore is enhanced while the external stray field is minimized. The magnets have a fixed dimension $d \times d \times 5 d$. To tune the homogeneity, the position of the magnets in the corners is fixed, while the other magnets are spread out over a distance $C$ (Fig. 2.7). The width starts at $W=3 d$ to ensure a minimum bore width $d$ and ends at $W=L$, due to the 


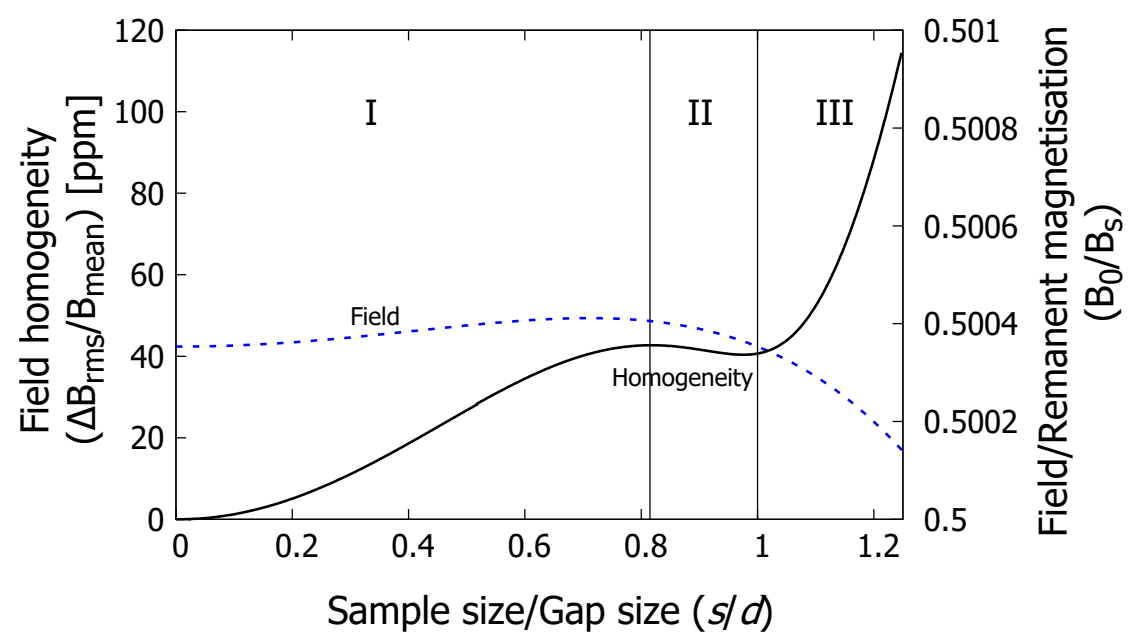

FIGURE 2.5 - Field inhomogeneity and field as a function of the sample size (length of interest) for the cuboid configuration. The homogeneity has been optimized for a sample size equal to the gap. With increasing sample size, both the field and the field inhomogeneity increase theoretically (region I). The field reaches a local maximum of $0.50041 B_{s}$ at a distance of $0.71 d$ from the center. Above this distance the homogeneity of the sample stays approximately the same (region II). When the sample size increases more than the gap size, the inhomogeneity strongly increases (region III). Hence, to optimize the homogeneity of the cuboid magnet configuration, the field difference between the center and the sample edge needs to be minimized.

previously chosen boundary conditions.

Spreading the configuration increases the distance of the middle magnets, which produces a decreased magnetic field strength (Fig. 2.7). With this configuration the convex field profile has no chance to change to a concave profile. Therefore a minimum can not be reached. With the most compact magnet arrangement, a field of $0.9 B_{\mathrm{S}}$ and a field difference of $3365 \mathrm{ppm}$ can be achieved. The stray field at a distance equal to the gap size from the surface is $0.07 B_{\mathrm{S}}$.

\section{Influence of variations in the magnets}

Choice of parameters to vary We calculated the influence on the magnetic field of a variation of the dimensions, position, and tilt of the magnets, as well as in the magnetization strength and angle (shown in Fig. 2.8). We consider the field 
Chapter 2 - Influence of the distribution of the properties of permanent magnets
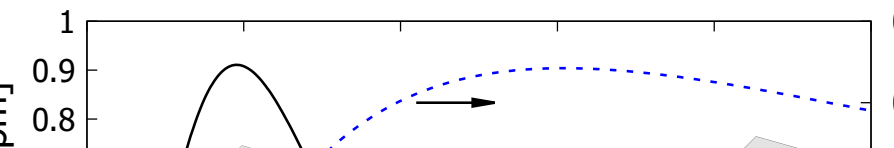

\section{$\uparrow$}

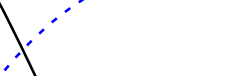
$\begin{aligned} & 0.4 \\ & 0.3 \\ & 0.2\end{aligned}-\therefore$

FIGURE 2.6 - Field strength in the middle of the configuration and difference of the field centre and the edge of the sample, both as functions of the ratio of the cuboid width over the length. The field increases up to $0.54 B_{s}$ at a cuboid width of 3.0375 times the gap size. The inset shows the field difference dropping to zero at a width/gap ratio of 4.72 .

components $B_{x}, B_{y}, B_{z}$ at a point above the centre of the top (north) face, at a height of $10 \%$ of the length $a$ of the edge of the magnet.

Besides calculations, we also measured the magnetization and magnetization angle of magnets with different sizes, materials, coatings, and manufacturers. These measured variations are used for Monte Carlo simulations to estimate the field homogeneity of a cuboid magnet and a Pseudo-Halbach magnet configuration after assembly.

As can be seen in Fig. 2.9, the cubic magnet, magnetized in the $z$-direction, shows no field in the $y$-direction $\left(B_{y}\right)$ along the $x$-axis at a distance of $0.1 a$ from the surface. The field $B_{x}$ is zero in the center of the magnet and rises linearly with a slope of $0.04 B_{\mathrm{S}} / a$ in the positive $x$-direction, with $B_{\mathrm{S}}$ the saturation magnetization of the magnet material [T]. The field $B_{z}$ is $0.357 B_{\mathrm{S}}$ in the centre which drops to $0.355 B_{\mathrm{S}}$ at $x=0.1 a$.

Table 2.3 shows the sensitivity matrix of the magnetic field in the $x, y$ and $z$ directions on the $x$-axis at a distance of $0.1 d$, given as percentages of $B_{\mathrm{s}}$. Table 2.4 and 2.5 show the sensitivity matrix of the cuboid and Pseudo-Halbach configuration, depending on the magnetization and angle variation of each individual magnet. Parameters related to the sizes have been varied by $10 \%$ of the length of the edge of the cube. Parameters related to the angle have been varied by $1^{\circ}$. The 


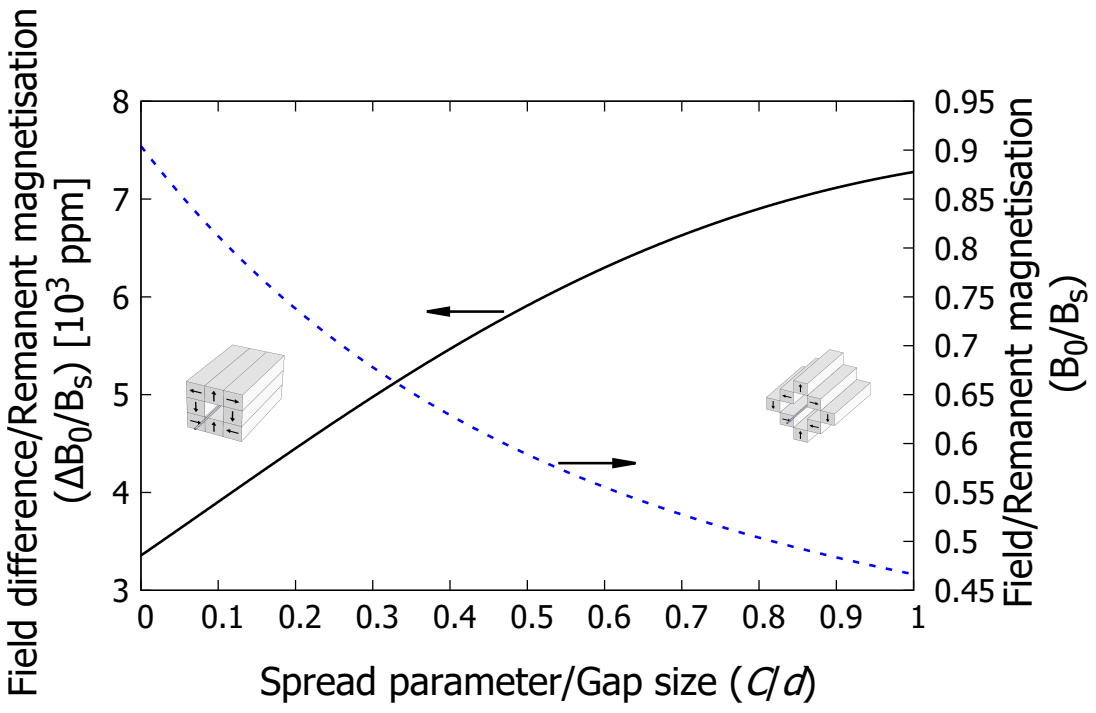

FIGURE 2.7 - Spreading the middle magnets has been used to change the normalized field strength and field difference. At a spread parameter $C=o, a$ minimal field difference of $3365 \mathrm{ppm}$ and a field strength of $0.90 B_{s}$ can be reached.

sensitivity matrix of the field in the $x$-direction shows that the field strength changes proportionally with the magnetization. The magnet tilt angle and the magnetization offset angle around the $x$-axis, as well as moving the magnet in the $y$-direction, does not have any influence on the magnetic field. A tilt or a magnetization offset angle around the $y$-axis has a much more significant influence on the field: tilting changes the field from $0.61 \%$ in the centre to $0.51 \%$ at $x=0.1 a$; the magnetic offset angle changes the field from $-0.87 \%$ in the centre to $-0.88 \%$ at $0.1 a$. Moving the magnet in the $x$-direction has the biggest influence on $B_{x}:-1.09 \%$ in the center to $-1.15 \%$ at $0.1 d$. Misplacements in other directions and variations in the dimensions have a minor influence on the field. The field in the $y$-direction is influenced by tilting the magnet and a magnetization offset angle around the $x$-axis and moving the magnet in the $y$-direction. The other parameters do not affect the field. The field in the $z$-direction has a high dependency on the distance to the magnet, twice as big as the change due to variations in the magnetization. Tilting the magnet changes the field linearly from 0 at $x=0$ to $-0.48 \%$ at $x=0.1 a$. Other variables have a rather small influence on the field.

Summarizing, the variations in the tilt and magnetization angle have a significant influence when the rotational axis is perpendicular to the observed axis. 


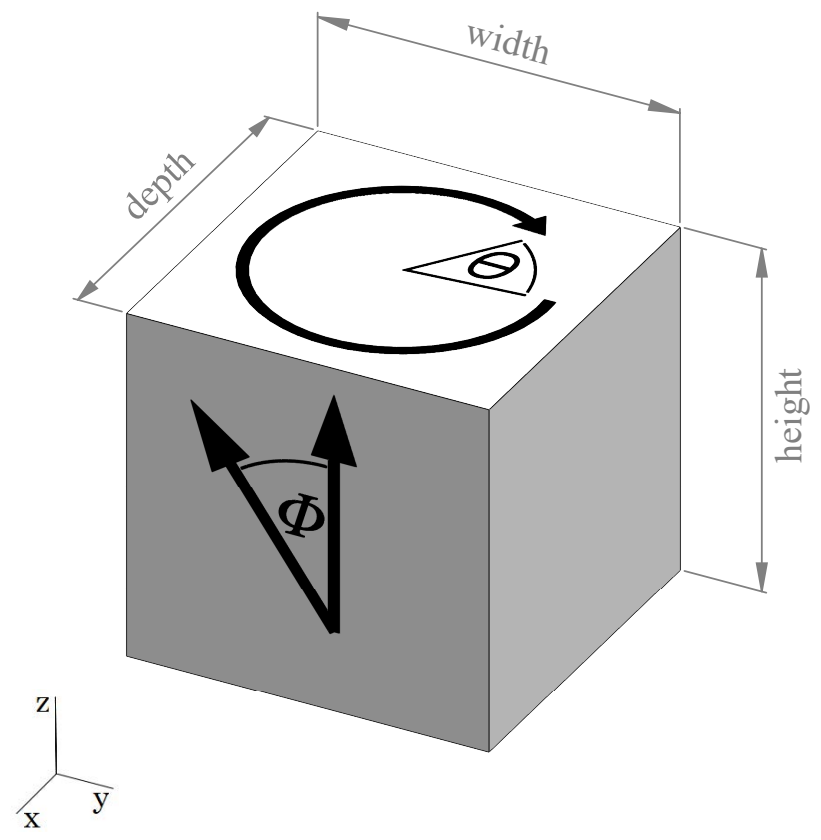

FIGURE 2.8 - Schematic drawing of a cubic magnet. The arrows indicate the direction of magnetization. $\phi$ shows the total offset angle, $\theta$ the offset direction in cylindrical coordinates.

Variations in the placement of the magnet have a significant influence if this variation is in the same direction as the observed field. Variations in the dimensions have a relatively small influence on the field at the observed locations compared to the other varied parameters.

Figure 2.10 shows a logarithmic plot of the field difference normalized to one-half of the sum of $B_{z}$ with an offset angle of $0^{\circ}$ and $1^{\circ}$ on the $x z$-plane. Right above ( $z$-axis) and next to the magnet ( $x$-axis), the field is least affected by the magnetization offset angle. The difference has its maximum along a line with slope $0.7 z / x$.

Simulation of effect of variations in the magnet A Monte Carlo simulation ( $n=50000)$ of the homogeneity has been performed with two input parameters for each magnet, both for the cuboid and the Pseudo-Halbach configuration. A normal distribution was assumed, using our measurements of the standard deviations of $1^{\circ}$ in the magnetization angle and $1 \%$ of the magnetization. No placement errors or dimensional errors were considered.

Fig. 2.11 shows a distribution and probability plot of the obtained homogeneities 


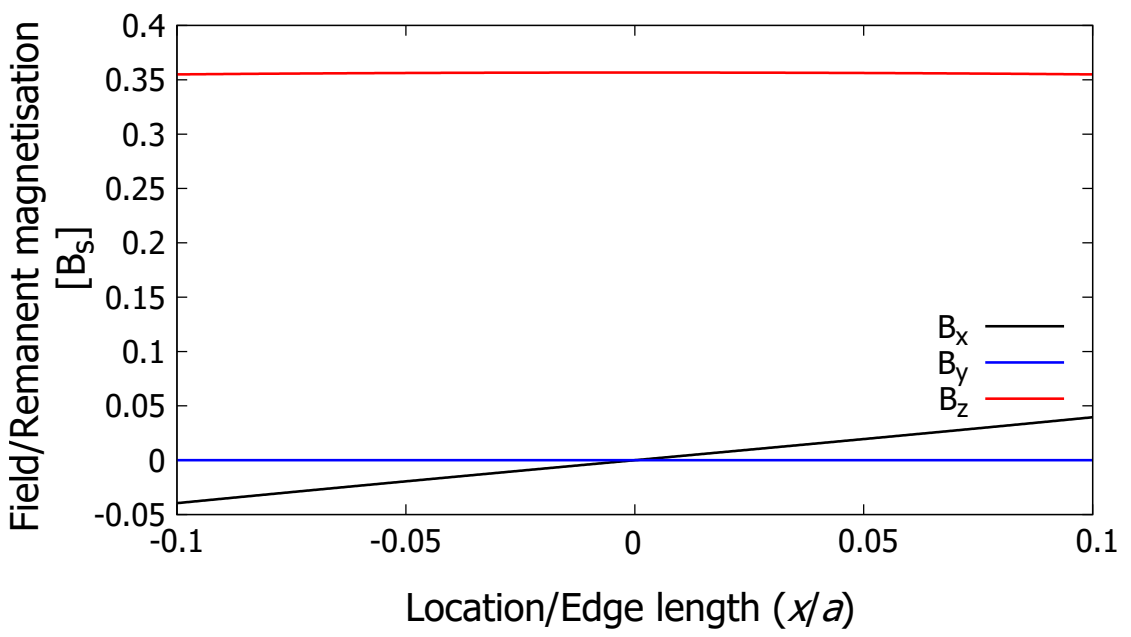

FIGURE 2.9 - Magnetic field $(x, y, z)$ above a cuboid magnet with the edge length $a$ along the $x$-axis at a distance of $0.1 a$.

TABLE 2.3 - Sensitivity matrix of the magnetic field ( $x, y, z)$, given in the change of $B_{s}$ at the same position in [\%] above a cuboid magnet with the edge length a along the $x$-axis at a distance of o.1a. Variations in the magnetization angle and tilting the magnet perpendicular to the simulated axis affect the magnetic field significantly. Placement errors have an influence if parallel to the field. Variations in the dimensions have a minor effect.

\begin{tabular}{|c|c|c|c|c|c|c|c|}
\hline & & \multicolumn{2}{|c|}{$B_{x}$} & \multicolumn{2}{|c|}{$B_{y}$} & \multicolumn{2}{|c|}{$B_{z}$} \\
\hline & \multicolumn{2}{|c|}{ Variation $x=0$} & $x=0.1 a$ & $x=0$ & $x=0.1 a$ & $x=0$ & $x=0.1 c$ \\
\hline$M$ & $1 \%$ & 0.00 & 1.10 & 0.00 & 0.00 & 1.00 & 0.99 \\
\hline tilt $x$ & $1^{\circ}$ & 0.00 & 0.00 & -0.61 & -0.62 & 0.00 & 0.00 \\
\hline tilt $y$ & $1^{\circ}$ & 0.61 & 0.51 & 0.00 & 0.00 & 0.00 & -0.48 \\
\hline$\phi\left(\theta=0^{\circ}\right)$ & $1^{\circ}$ & 0.00 & 0.00 & 0.00 & 0.00 & 0.00 & 0.00 \\
\hline$\phi\left(\theta=90^{\circ}\right)$ & $1^{\circ}$ & -0.87 & -0.88 & -0.87 & -0.86 & 0.00 & 0.19 \\
\hline$x$ & $0.1 a$ & -1.09 & -1.15 & 0.00 & 0.00 & 0.00 & 0.10 \\
\hline$y$ & $0.1 a$ & 0.00 & 0.00 & 1.09 & 1.07 & 0.00 & 0.00 \\
\hline$z$ & $0.1 a$ & 0.00 & -0.10 & 0.00 & 0.00 & -2.17 & -2.22 \\
\hline height & $0.1 a$ & 0.00 & 0.02 & 0.00 & 0.00 & 0.23 & 0.23 \\
\hline depth & $0.1 a$ & 0.00 & -0.18 & 0.00 & 0.00 & -0.01 & 0.01 \\
\hline width & $0.1 a$ & 0.00 & 0.05 & 0.00 & 0.00 & -0.01 & -0.01 \\
\hline
\end{tabular}




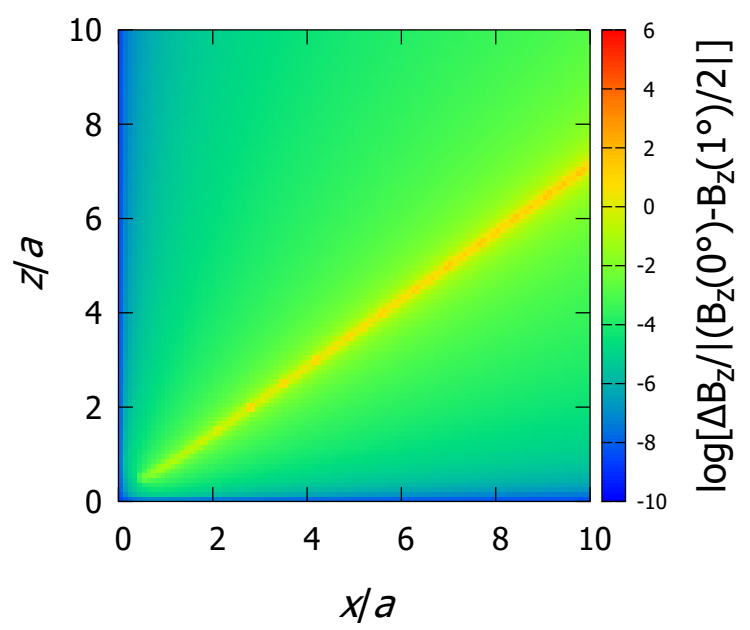

FIGURE 2.10 - Logarithmic plot of the field difference normalized to one-half of the sum of $B_{z}$ with an offset angle of $o^{\circ}$ and $1^{\circ}$ generated by a cubic magnet with an edge length of $a$. The field difference has its minimum right above, and right next to the magnet, along the $x$-and $z$-axes. A maximum can be found along a straight line with a slope of $0.7 z / x$.

of the magnetic field in the $z$-direction simulated by a Monte Carlo simulation. The mean homogeneity of the cuboid configuration is $430 \mathrm{ppm}$, the Pseudo-Halbach configuration achieves $1086 \mathrm{ppm}$. However, the cuboid configuration has a high spread in the homogeneity (standard deviation $350 \mathrm{ppm}$ ) while the Pseudo-Halbach has a standard deviation of only $8 \mathrm{ppm}$. With a probability of $94.4 \%$, both the cuboid configuration and the Pseudo-Halbach configuration obtain a homogeneity of $1098 \mathrm{ppm}$ or better. With a probability of $10 \%$, the cuboid configuration achieves $64 \mathrm{ppm}$ whereas the Pseudo-Halbach achieves not less than $1076 \mathrm{ppm}$.

An indication of why the cuboid configuration has a much higher standard deviation than the Pseudo-Halbach configuration can be seen from the sensitivity matrices of the $z$-field. We chose to show how the field in the centre and at $x=d / 2$ changes for a magnetization difference of $1 \%$ and an offset magnetization direction of $1^{\circ}$ each in the direction which creates the highest field difference at both locations. The Halbach configuration consists of 8 magnets: 4 corner magnets, 2 at the side, and 1 each on top and bottom. Adding up the sensitivity values of all the magnets results in a difference of $314 \mathrm{ppm}$ between the $z$-field at $x=0$ and $x=d / 2$. The cuboid shows a significantly higher difference of $1970 \mathrm{ppm}$.

Regarding a single cubic magnet, the field difference, normalized to onehalf the sum of $B_{z}$, has a minimum right above and next to the magnet. The 

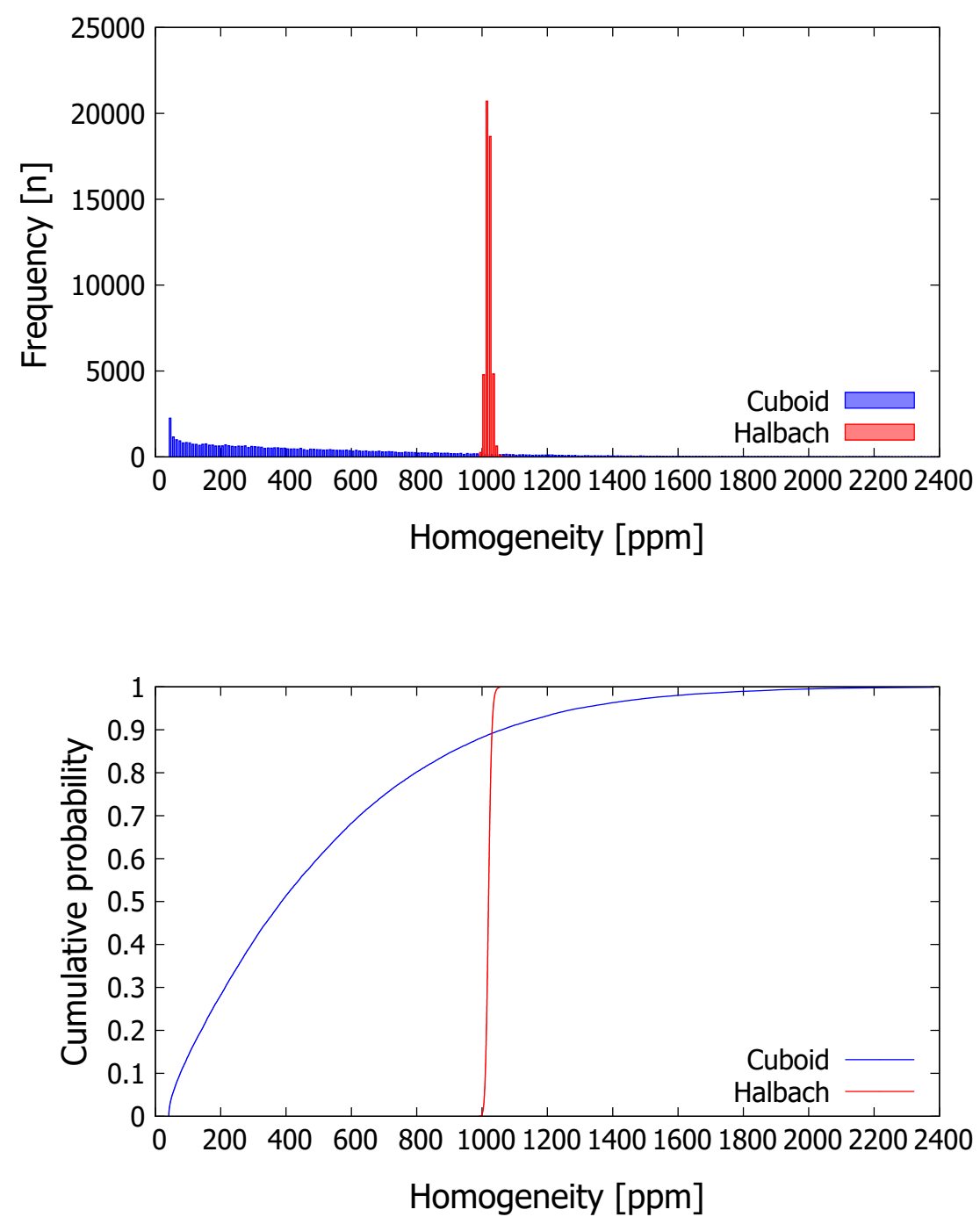

FIGURE 2.11 - Density plot (top) and cumulative distribution function (bottom) of the Monte Carlo simulation for the cubic and Pseudo-Halbach configurations.

The simulation shows that the Pseudo-Halbach configuration has a mean homogeneity of $1086 \mathrm{ppm}$ (standard deviation $8 \mathrm{ppm}$ ), while the cuboid configuration reaches $430 \mathrm{ppm}$ (standard deviation $350 \mathrm{ppm}$ ). 
Chapter 2 - Influence of the distribution of the properties of permanent magnets 20 on the field homogeneity of magnet assemblies for mobile NMR

TABLE 2.4 - Sensitivity matrix of the $z$-field at $x=o$ and $x=d / 2$ for the Pseudo-Halbach configuration.

\begin{tabular}{lccl}
\hline & $B_{z}(x=0)$ & $B_{z}(x=d / 2)$ & \\
\hline$M$ (total) & 902.237 & 899.209 & $\mathrm{mT}$ \\
$M$ (top/bottom) & 1737 & 1743 & $\mathrm{ppm}$ \\
$M$ (side) & 1515 & 1520 & $\mathrm{ppm}$ \\
$M$ (corner) & 876 & 877 & $\mathrm{ppm}$ \\
Angle (top/bottom) & 0 & 59 & $\mathrm{ppm}$ \\
Angle (side) & 0 & 0 & $\mathrm{ppm}$ \\
Angle (corner) & 0 & 81 & $\mathrm{ppm}$ \\
\hline \hline
\end{tabular}

TABLE $2.5-$ Sensitivity matrix of the $z$-field at $x=o$ and $x=d / 2$ for the cuboid-configuration.

\begin{tabular}{lccl}
\hline \hline & $B_{z}(x=0)$ & $B_{z}(x=d / 2)$ & \\
\hline$M$ (total) & 499.301 & 499.301 & $\mathrm{mT}$ \\
$M$ & 4998 & 4998 & $\mathrm{ppm}$ \\
Angle & 0 & 985 & $\mathrm{ppm}$ \\
\hline \hline
\end{tabular}

TABLE 2.6 - Measured homogeneity of cuboid and Pseudo-Halbach configurations.

\begin{tabular}{lc}
\hline \hline & $\begin{array}{c}\text { Inhomogeneity } \\
\text { [ppm] }\end{array}$ \\
\hline Cuboid 1 & $748(3)$ \\
Cuboid 2 & $2250(3)$ \\
Cuboid 3 & $1021(3)$ \\
Pseudo-Halbach 1 & $1088(3)$ \\
Pseudo-Halbach 2 & $1081(3)$ \\
Pseudo-Halbach 3 & $929(3)$ \\
\hline \hline
\end{tabular}

difference increases with an increased distance from the axis. Since the cuboid configuration has fewer of magnets, the chance that all magnets have the same deviation in their magnetization angle in the same direction is much higher than for the Pseudo-Halbach configuration.

Measurements of the effect of variations in the magnet on the homogeneity, for the cuboid and the Pseudo-Halbach configurations Both configurations were assembled and measured three times. The measurement results are shown in Table 2.6. One can see a small spread in the homogeneity of the Pseudo-Halbach (mean value of $1032 \mathrm{ppm}$ and standard deviation of $90 \mathrm{ppm}$ ). A larger spread was found for the cuboid configuration (1340 and $800 \mathrm{ppm}$ ), see Table 2.7.

Using the results of the Monte Carlo simulation from Fig. 2.12, we can calculate the likelihood of measuring a homogeneity of, for instance, approximately 
TABLE 2.7 - Mean (standard deviation) of the homogeneity of the field in the $z$-direction of measured and simulated magnet configurations.

\begin{tabular}{lcc}
\hline \hline & $\begin{array}{c}\text { Measured } \\
\text { Inhomogeneity } \\
{[\mathrm{ppm}]}\end{array}$ & $\begin{array}{c}\text { Simulated } \\
\text { Inhomogeneity } \\
{[\mathrm{ppm}]}\end{array}$ \\
\hline Cuboid & $1340(800)$ & $430(350)$ \\
Pseudo-Halbach & $1032(90)$ & $1086(6)$ \\
\hline \hline
\end{tabular}

TABLE 2.8 - Comparison of magnetic properties of different magnet configurations.

\begin{tabular}{lccc}
\hline \hline & $\begin{array}{c}B_{\max } \\
{\left[B_{\mathrm{S}}\right]}\end{array}$ & $\begin{array}{c}B_{\text {stray }} \\
{\left[B_{\mathrm{S}}\right]}\end{array}$ & $\begin{array}{c}\Delta B_{\mathrm{rms}} / B_{\text {mean }} \\
{[\mathrm{ppm}]}\end{array}$ \\
\hline Cuboid & 0.5 & 0.24 & 41 \\
Pseudo-Halbach & 0.9 & 0.07 & 994 \\
\hline \hline
\end{tabular}

$1000 \mathrm{ppm}$ or worse. For the cuboid configuration, this is a relatively low likelihood of $6.5 \%$, and even lower $(0.006 \%)$ for the Pseudo-Halbach configuration. However, we measured inhomogeneities above $1000 \mathrm{ppm}$ on only three realizations. It seems very likely that the inhomogenities are not only caused by the spread in the properties of the magnets themselves, but also by inaccuracies in the manufacturing of the entire assembly.

In general, the Pseudo-Halbach configuration has a more predictable field profile, which makes this design more favorable for industrial applications than the cuboid configuration. Since shimming is needed anyway, a measurement of the field profile is not necessary. We therefore recommend restricting the use of the cuboid configurations to research systems, where selecting the magnets and measuring the final assembly is feasible.

\subsubsection{Comparison}

\section{General comparison}

Table 2.8 compares the major specifications of the two configurations. The Pseudo-Halbach configuration achieves $0.9 B_{\mathrm{s}}$, a 1.8 times higher field than the Cuboid configuration, while the stray field at a distance of $d$ from the magnet surface is $0.07 B_{\mathrm{s}}$, which is 3.4 times lower. In terms of homogeneity, the cuboid configuration achieves a homogeneity of $41 \mathrm{ppm}$, which, compared to the PseudoHalbach configuration, is 24.2 times better.

\section{Influence of the sample length}

We optimized the homogeneity of the configuration for different sample sizes, while keeping the outer boundary conditions the same. Fig. 2.12 shows how the 
Chapter 2 - Influence of the distribution of the properties of permanent magnets

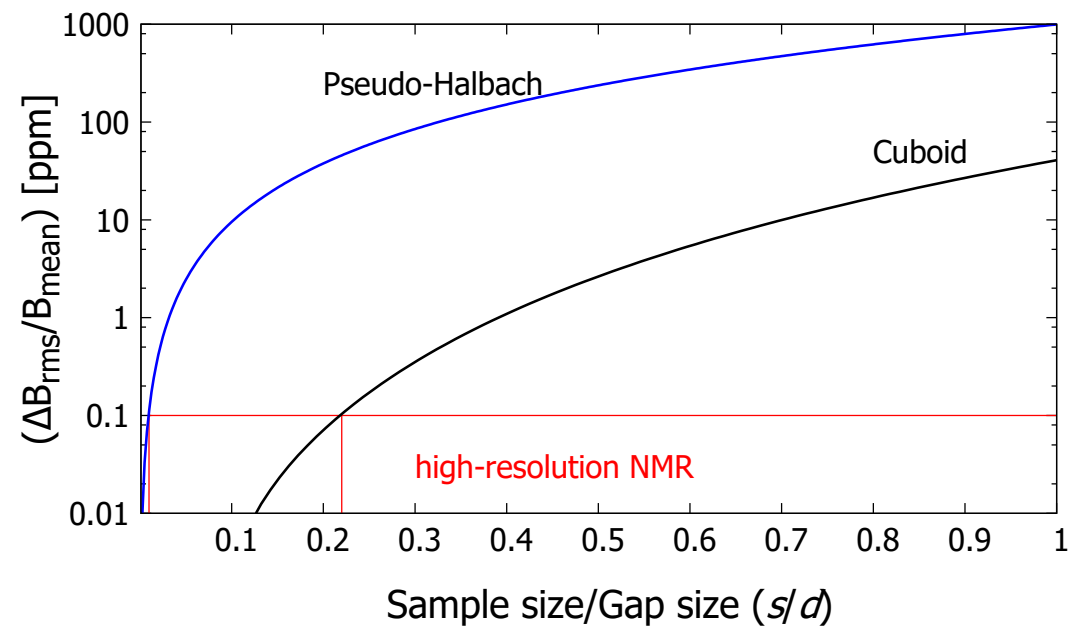

FIGURE 2.12 - Inhomogeneity of the magnetic field as a function of the sample length/gap ratio with a constant configuration width/gap ratio of 5. For every sample size, the width of the cuboid configurations has been optimized to reach the lowest possible inhomogeneity. For a homogeneity reasonable for NMR applications of o.1 ppm, the sample length for a cuboid configuration needs to be $0.22 d$, whereas it has to become unrealistically short (o.o1d) for the Pseudo-Halbach configuration.

homogeneity changes with a smaller ratio of the gap size to the sample size.

In every case, reducing the sample size yields a better homogeneity. The cuboid configuration can reach in theory $0.01 \mathrm{ppm}$ with a sample size of $0.22 d$, while the Pseudo-Halbach configuration needs an absurd sample length of $0.01 d$ to reach this value.

\subsection{Conclusions}

We have investigated the effect on the homogeneity of the field of permanent magnet configurations for mobile NMR applications of variations in the properties of the magnets. We measured the variations in the magnetization and magnetization angle of permanent magnets but could not observe a decisive difference between the manufacturers, materials, or magnet coatings. On average, the standard deviation of the magnetization is less than $1 \%$ and for the variations in the magnetization angle it is less than $1^{\circ}$.

We compared a cuboid and a Pseudo-Halbach magnet configuration, in terms of 
their field strength and field homogeneity, for our optimized boundary conditions, in which the sample size is equal to the gap size $d$ and the whole configuration should fit in a box with an edge length five times the gap size. For a fixed parameter set, assuming perfectly magnetized magnets, the field in the centre of the cuboid configuration is $0.5 B_{\mathrm{s}}$ and its homogeneity is $41 \mathrm{ppm}$. For the same boundary conditions, the Pseudo-Halbach configuration achieves a higher magnetization $\left(0.9 B_{\mathrm{s}}\right)$ in the centre but less homogeneity $(994 \mathrm{ppm})$. It is worth mentioning that the Pseudo-Halbach configuration has a much lower stray field, and so less interference with the environment, than the cuboid configuration.

For samples with a size the same as the gap size, the theoretical homogeneity of both configurations is above the sub-ppm range, which is necessary to produce a high resolution spectrum. Optimizing the homogeneity for shorter samples while respecting the maximum outer dimensions yields in a much better homogeneity. Using a sample size of $0.22 d$ improves the homogeneity from 41 to $0.1 \mathrm{ppm}$ for the cuboid configuration, whereas the Pseudo-Halbach configuration would need a impractical sample size of $0.01 d$.

We analysed the effect of the variation in magnetic properties on the uniformity of the generated fields. The sensitivity matrix shows that the magnetization, magnetization angle, and tilt have the most significant influence on the magnetic field. Positioning errors mainly change the field, in case the positioning variation is in the same direction as the field. Theoretically, the cuboid has good homogeneity (on average $430 \mathrm{ppm}$ ), but the effect of variation in the magnets' properties is large (standard deviation $350 \mathrm{ppm}$ ). The Pseudo-Halbach configuration has worse homogeneity ( $1080 \mathrm{ppm})$, but is 44 times less sensitive to variation in the properties of the magnet.

We advise using the cuboid configuration for scientific use, where it is possible to preselect the permanent magnets and the external stray field is not a big issue. Mechanical shimming of this configuration can be done, changing the distance to the magnets (counteracting magnetization differences) or by tilting the magnet (counteracting magnetization angle variations). Using rather large magnets helps to achieve the homogeneity needed for NMR measurements. If preselecting the magnets is not an option, we recommend the Pseudo-Halbach configuration, which has a more robust homogeneity regarding variations in the magnetization and angle. The field profile of this configuration is predictable, which makes it easier to shim afterwards to achieve the field homogeneity needed for NMR applications. Also the lower stray field makes this configuration easier to handle and therefore more favourable especially for industrial applications. 
Chapter 2 - Influence of the distribution of the properties of permanent magnets 24

\subsection{Appendix}

\subsubsection{Figures}

Figure 2.13 shows the offset angle from the same magnet, which has been measured 50 times resulting in a standard deviation of $0.645^{\circ}$, figs. 2.14 to 2.19 shows the histogram of the angle variation of 50 different magnets of the same type of respectively $\mathrm{HK}_{7} \mathrm{Nd}_{35} \mathrm{~N}, \mathrm{HK}_{7} \mathrm{Nd}_{35}, \mathrm{HK}_{7} \mathrm{Sm}_{2} 8 \mathrm{~N}, \mathrm{Su}_{7} \mathrm{Nd}_{42} \mathrm{NC}, \mathrm{Su}_{7} \mathrm{Nd} 42 \mathrm{NCN}$, $\mathrm{Su} 45^{\mathrm{Nd}} 45_{\mathrm{NCN}}$.

This data is used in table 2.2.

Figure 2.20 shows the cumulative distribution function plot for a sample with the length $d$ and $2 d$ in the cuboid configuration. The width of the cuboids with a distance of $d$ and a height of $2 d$ have been optimized for a sample length of $d$ (blue) and a sample length of $2 d$ (red).

Figure 2.21 shows the measured magnetic field $(z)$ of Cuboid and PseudoHalbach configuration along the $x$-axis for $d=8 \mathrm{~mm}$.

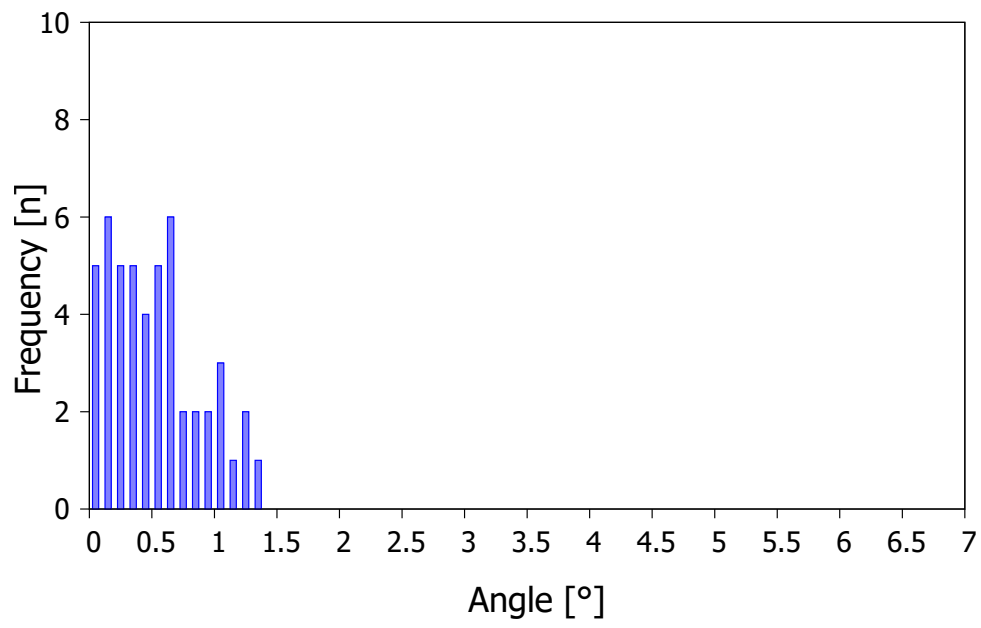

FIGURE 2.13 - The offset angle from the same magnet has been measured 50 times resulting in a standard deviation of $0.645^{\circ}$. 


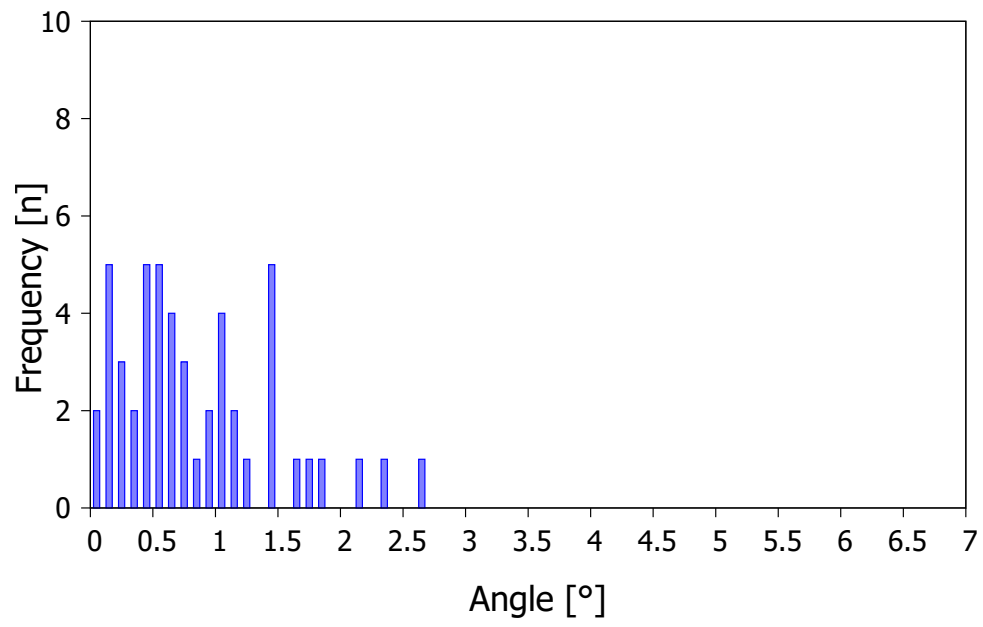

FIGURE 2.14 - Histogram angle variation $\mathrm{HK}_{7} \mathrm{Nd} 35 \mathrm{~N}$.

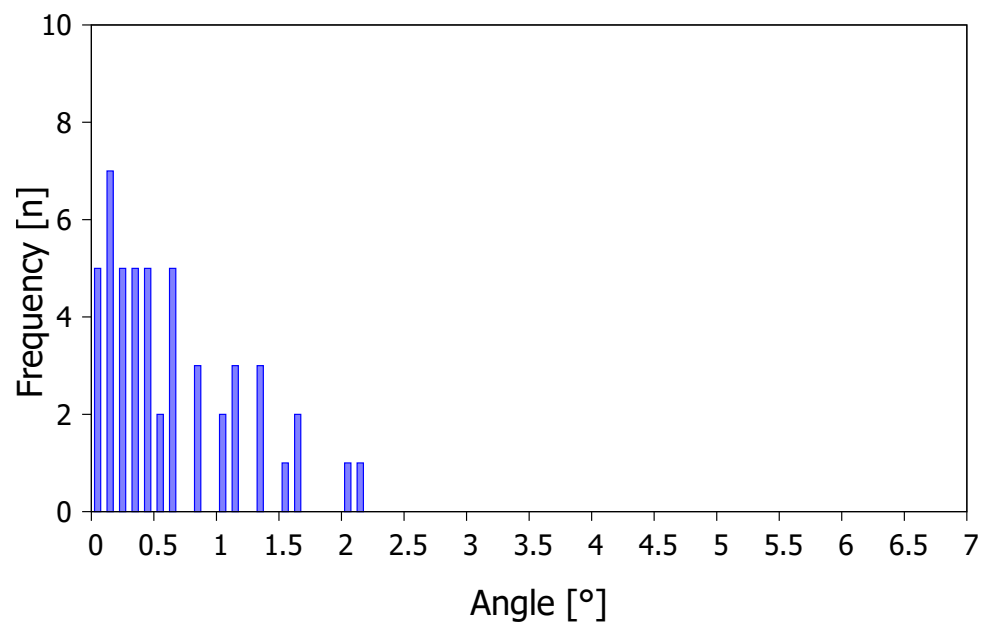

FIGURE 2.15- Histogram angle variation $\mathrm{HK}_{7} \mathrm{Sm} 28 \mathrm{~N}$. 
Chapter 2 - Influence of the distribution of the properties of permanent magnets 26 on the field homogeneity of magnet assemblies for mobile NMR

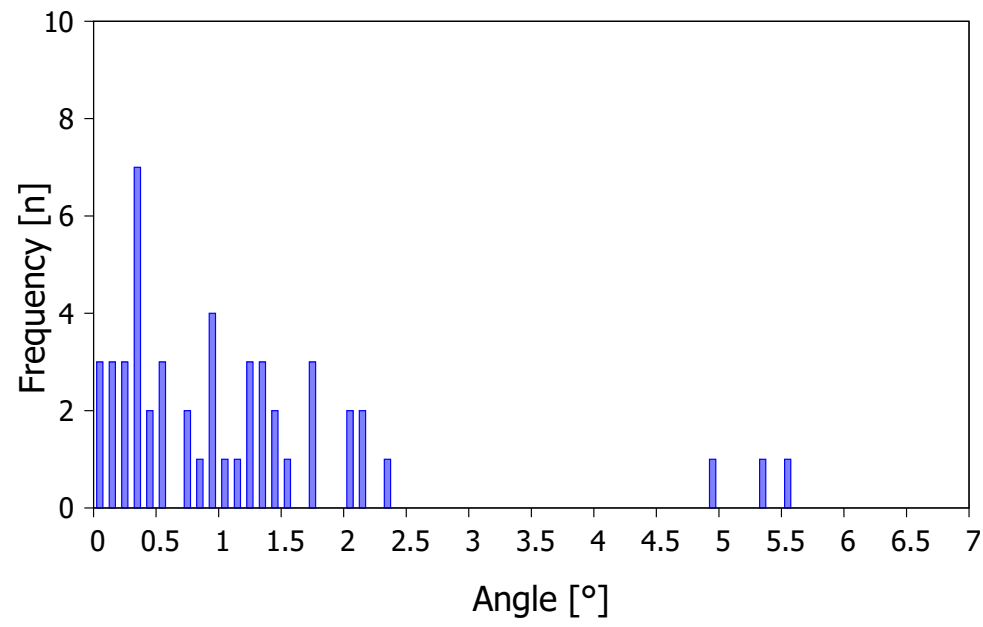

FIGURE 2.16 - Histogram angle variation Sc 7 Sm 26.

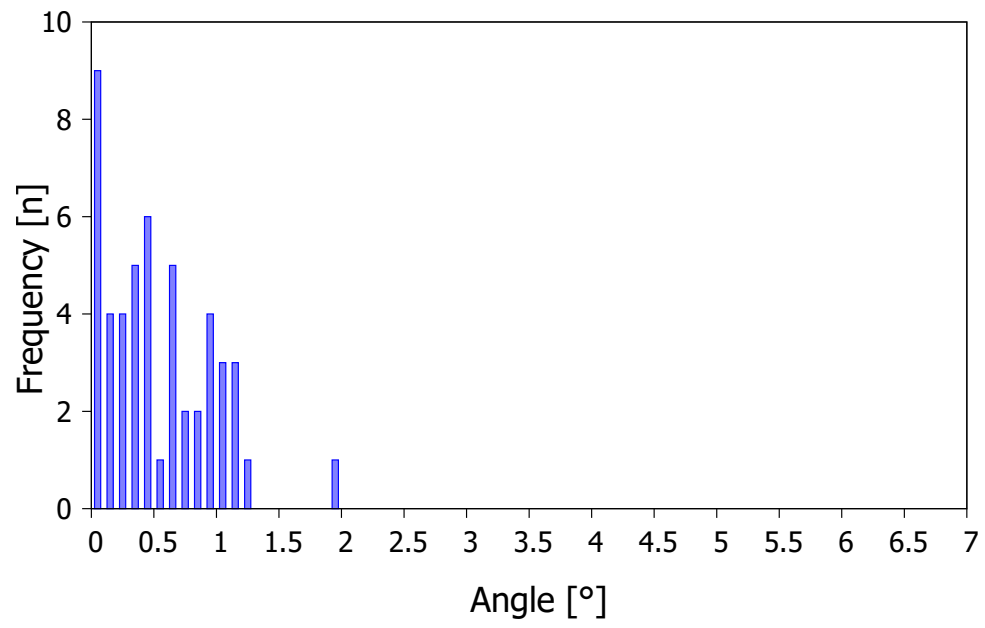

FIGURE 2.17 - Histogram angle variation $\mathrm{Su}_{7} \mathrm{Nd}_{42} \mathrm{NC}$. 


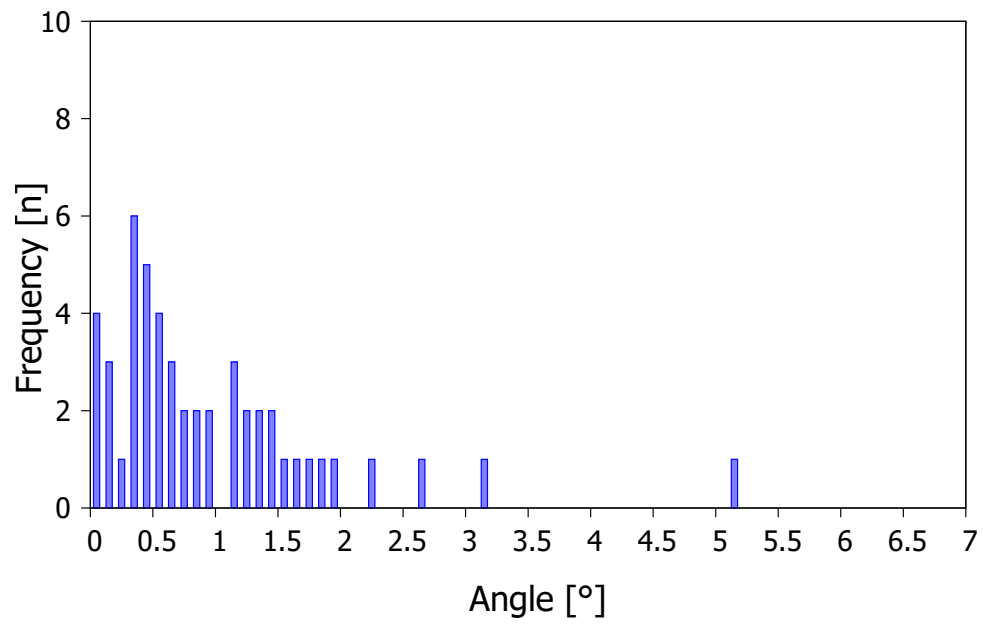

FIGURE 2.18 - Histogram angle variation $\mathrm{Su}_{7} \mathrm{Nd} 42 \mathrm{NCN}$.

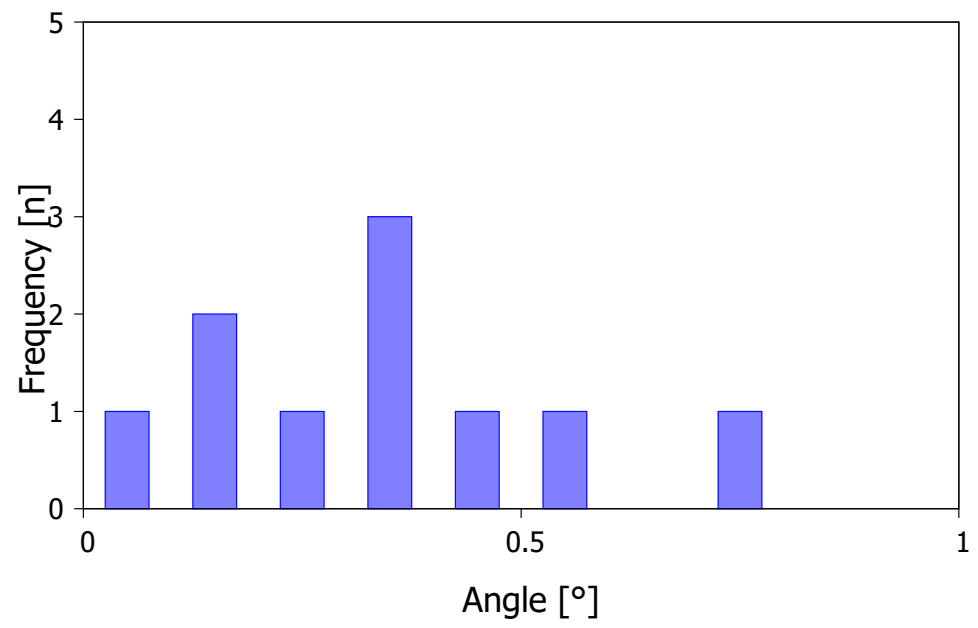

FIGURE 2.19- Histogram angle variation Su $45{ }^{N} d 4{ }_{5} \mathrm{NCN}$. 
Chapter 2 - Influence of the distribution of the properties of permanent magnets 28 on the field homogeneity of magnet assemblies for mobile NMR

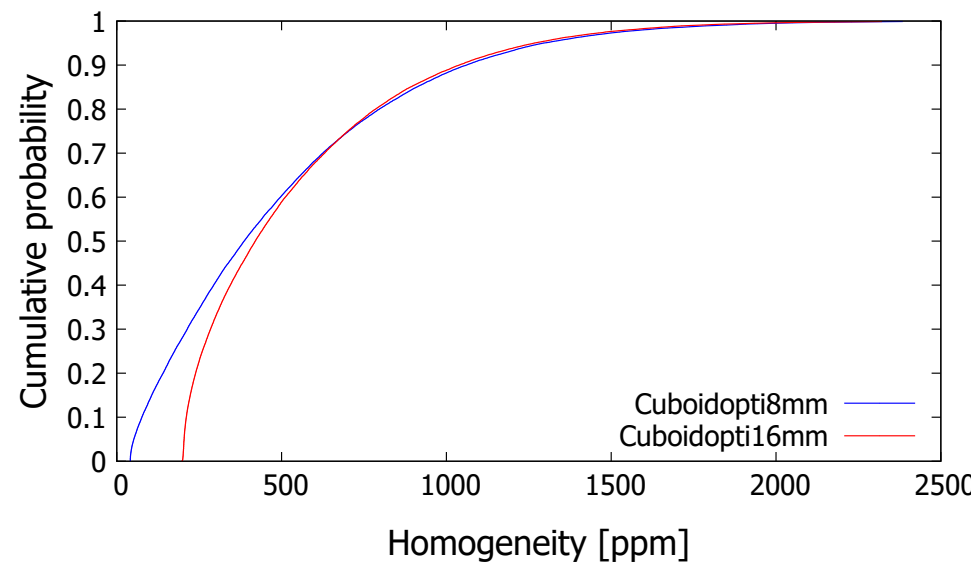

FIGURE 2.20-Cumulative distribution function plot for a sample with the length $d$. The width cuboids with a distance of $d$ and a height of $2 d$ have been optimized for a sample length of $d$ (blue) and a sample length of $2 d$ (red).

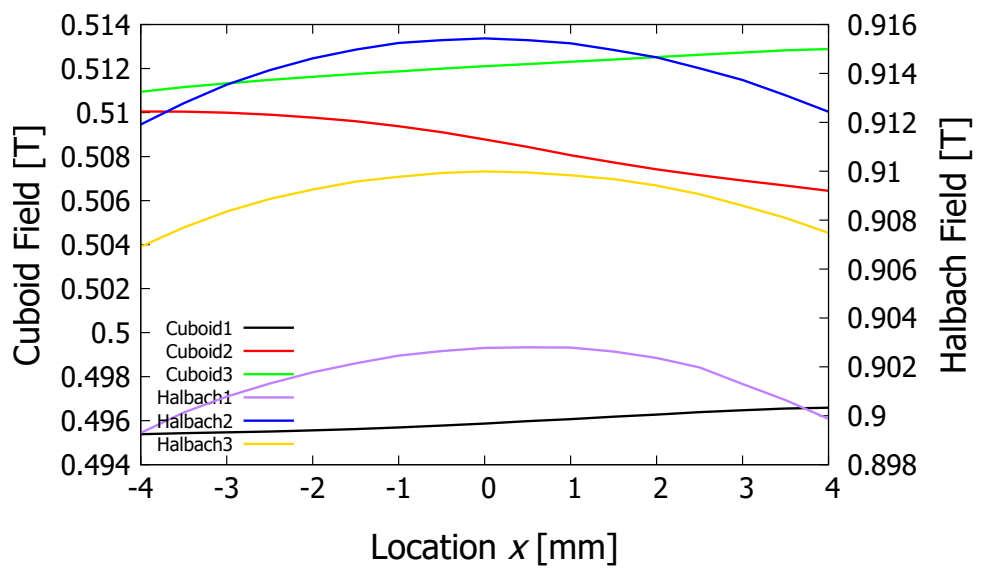

FIgURE 2.21 - Measured magnetic field (z) of Cuboid and Pseudo-Halbach configuration along $x$-axis. 


\subsubsection{Tables}

Table tables 2.9 and 2.10 shows measured angle and magnetization variations.

Table tables 2.11 to 2.13 shows the sensitivity matrix of $B_{x}, B_{y}, B_{z}$ above a cuboid magnet with the edge length $d$ along $x$-axis at a distance of $0.1 d$

TABLE 2.9-Measured angle variations.

\begin{tabular}{|c|c|c|c|c|c|}
\hline & \multirow[b]{2}{*}{ Material } & \multirow[b]{2}{*}{ Coating } & \multirow[b]{2}{*}{$\begin{array}{l}\text { std } \\
{\left[{ }^{\circ}\right]}\end{array}$} & \multicolumn{2}{|c|}{ confidence interval } \\
\hline & & & & $95 \%$ & $68.27 \%$ \\
\hline $\begin{array}{l}\text { Measurement } \\
\text { error }\end{array}$ & NdFeB N42 & $\mathrm{Ni}-\mathrm{Cu}-\mathrm{Ni}$ & 0.65 & 0.11 & 0.06 \\
\hline Supermagnete & $\mathrm{NdFeB} \mathrm{N} 42_{4}$ & $\mathrm{Ni}-\mathrm{Cu}-\mathrm{Ni}$ & 1.33 & 0.2 & 0.11 \\
\hline Supermagnete & $\mathrm{NdFeB} \mathrm{N} 42$ & $\mathrm{Ni}-\mathrm{Cu}$ & 0.69 & 0.113 & 0.06 \\
\hline HKCM & NdFeB N35 & $\mathrm{Ni}$ & 1.05 & 0.17 & 0.09 \\
\hline HKCM & $\begin{array}{l}\mathrm{Sm}_{2} \mathrm{Co} 17 \\
\mathrm{YXG} 28\end{array}$ & $\mathrm{Ni}$ & 0.85 & 0.14 & 0.07 \\
\hline $\begin{array}{l}\text { Schallenkammer } \\
\text { Magnetsysteme }\end{array}$ & $\begin{array}{l}\mathrm{Sm} 2 \mathrm{Co} 17 \\
\text { YXG-26H }\end{array}$ & - & 1.72 & 0.28 & 0.14 \\
\hline $\begin{array}{l}\text { Supermagnete } \\
\text { (Cylinder) }\end{array}$ & $\mathrm{NdFeB} \mathrm{N} 45$ & $\mathrm{Ni}-\mathrm{Cu}-\mathrm{Ni}$ & 0.42 & 0.13 & 0.07 \\
\hline
\end{tabular}

TABLE 2.10 - Measured magnetization variations.

\begin{tabular}{|c|c|c|c|c|c|}
\hline & Material & Coating & $\mathrm{B}[\mathrm{T}]$ & $\sigma$ & $\begin{array}{l}68.27 \% \\
\text { confidence }\end{array}$ \\
\hline $\begin{array}{l}\text { Measurement } \\
\text { error }\end{array}$ & $\mathrm{NdFeB} \mathrm{N} 42$ & $\mathrm{Ni}-\mathrm{Cu}-\mathrm{Ni}$ & 0.5425 & 0.1709 & 0.0002 \\
\hline Supermagnete & $\mathrm{NdFeB} \mathrm{N} 42$ & $\mathrm{Ni}-\mathrm{Cu}-\mathrm{Ni}$ & 0.4196 & 0.8631 & 0.1374 \\
\hline Supermagnete & $\mathrm{NdFeB} \mathrm{N} 42_{4}$ & $\mathrm{Ni}-\mathrm{Cu}$ & 0.5187 & 0.7965 & 0.0669 \\
\hline HKCM & NdFeB N35 & $\mathrm{Ni}$ & 0.4503 & 0.9208 & 0.0773 \\
\hline HKCM & $\begin{array}{l}\mathrm{Sm}_{2} \mathrm{Co}_{17} \\
\mathrm{YXG}_{2} 8\end{array}$ & $\mathrm{Ni}$ & 0.4362 & 0.5025 & 0.0422 \\
\hline $\begin{array}{l}\text { Schallenkammer } \\
\text { Magnetsysteme }\end{array}$ & $\begin{array}{l}\mathrm{Sm} 2 \mathrm{Co} 17 \\
\mathrm{YXG}-26 \mathrm{H}\end{array}$ & - & 0.3852 & 1.2024 & 0.1010 \\
\hline $\begin{array}{l}\text { Supermagnete } \\
\text { (Cylinder) }\end{array}$ & $\mathrm{NdFeB} \mathrm{N} 45$ & $\mathrm{Ni}-\mathrm{Cu}-\mathrm{Ni}$ & 0.3687 & 1.7225 & 0.1447 \\
\hline
\end{tabular}


Chapter 2 - Influence of the distribution of the properties of permanent magnets 30

$$
\begin{aligned}
& \text { on the field homogeneity of magnet assemblies for mobile NMR }
\end{aligned}
$$

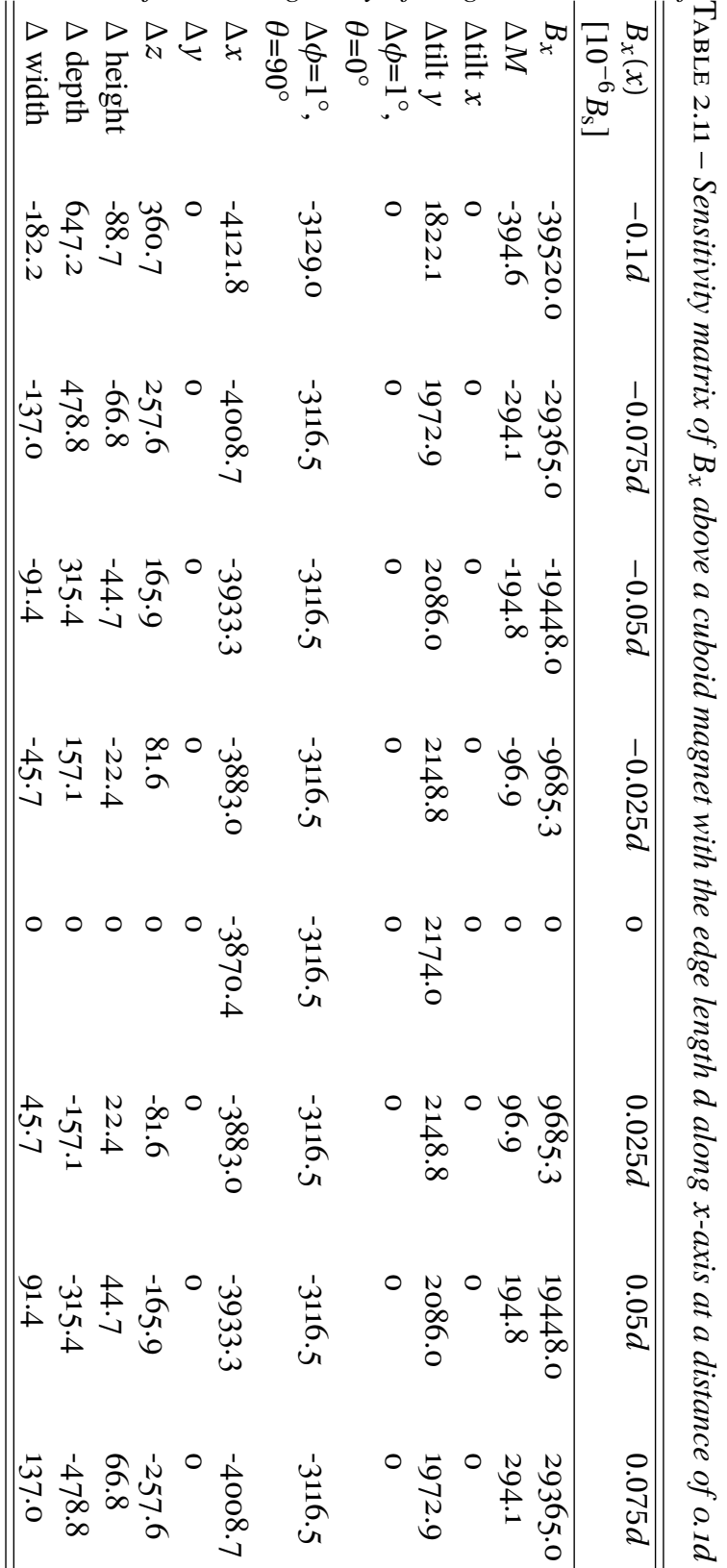

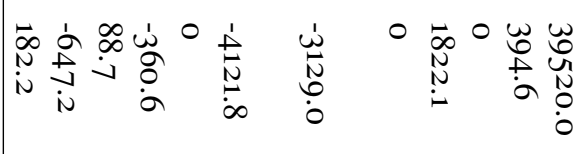




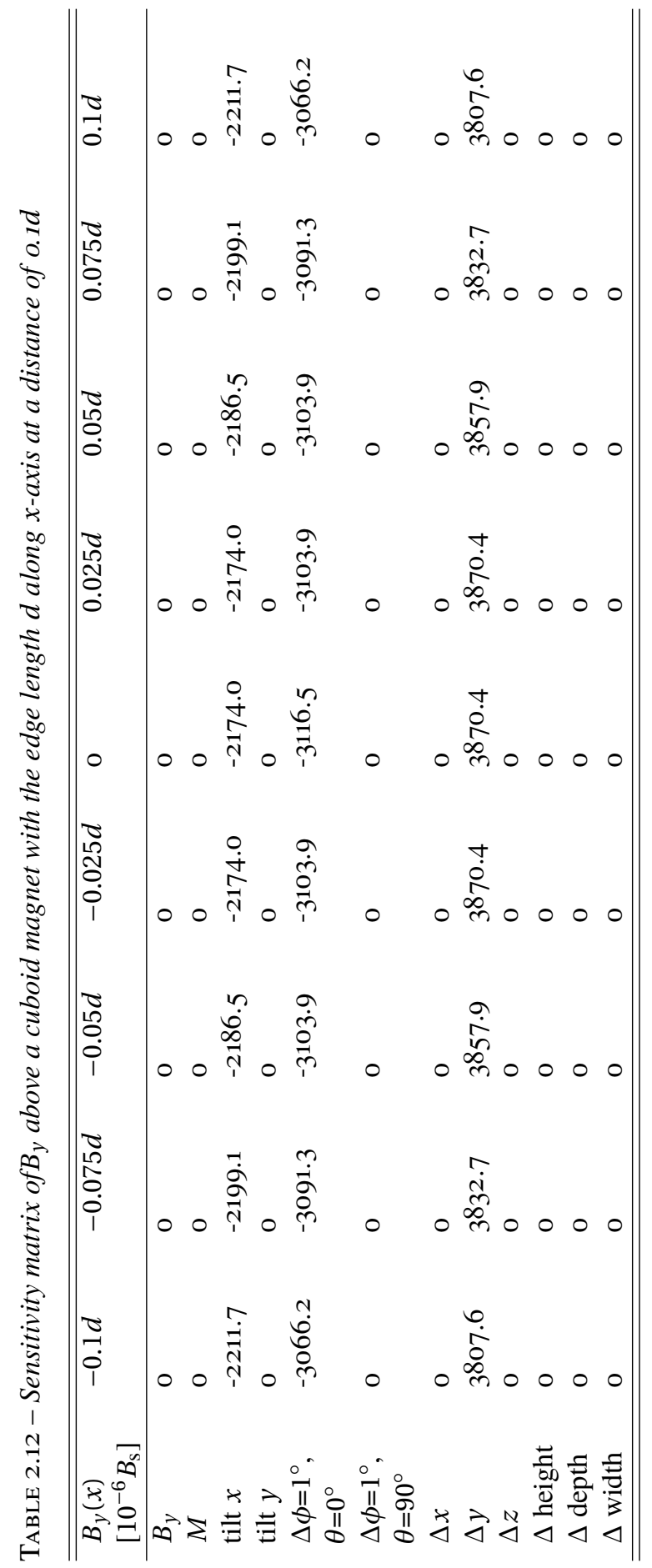


Chapter 2 - Influence of the distribution of the properties of permanent magnets 32

$$
\begin{aligned}
& \text { on the field homogeneity of magnet assemblies for mobile NMR }
\end{aligned}
$$

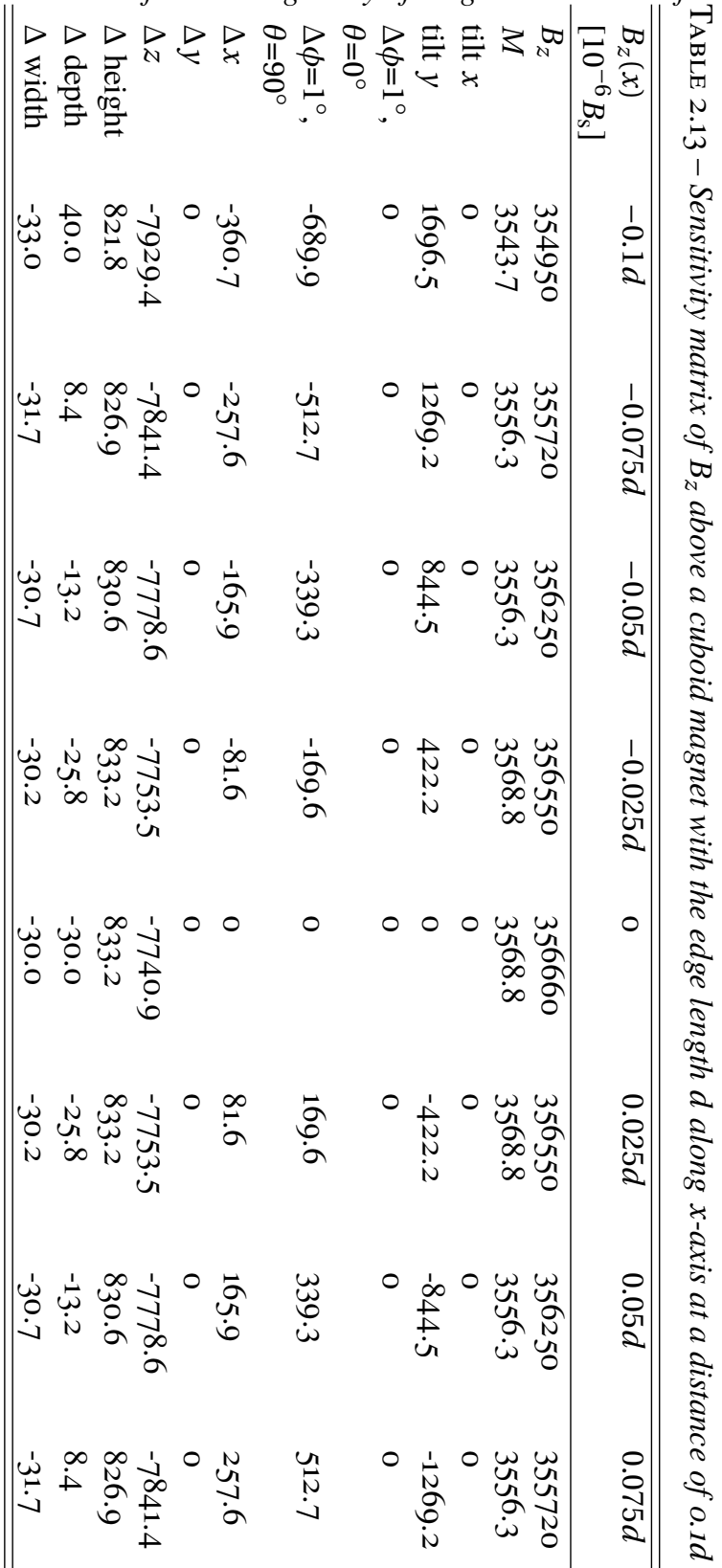

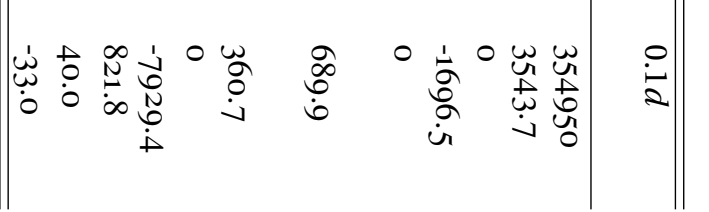




\title{
Chapter 3
}

\section{Dumbbell Pseudo Halbach Magnet Configuration: Combining high field strength with high homogeneity}

\begin{abstract}
In this work we introduce an easy-to-use permanent magnet configuration for mobile NMR-applications (nicknamed Dumbell-Halbach). The configuration consists out of a Pseudo-Halbach and two movable shimming rings which allow to tune the homogeneity along the bore to match it with the sample length. The two additional shimming-rings result in a $19 \%$ higher magnetic field strength and an improved homogeneity. Our assembled magnet has a total size of $50 \mathrm{~mm} \times 50 \mathrm{~mm} \times 42 \mathrm{~mm}$, a total weight of $332 \mathrm{~g}$ and reaches $33 \mathrm{ppm}$ and $1.06 \mathrm{~T}$ without electric shimming over a length of $5 \mathrm{~mm}$. Additionally, we present biplanar shimming circuits to shim magnetic field profiles on small scale. These circuits were optimized for the Dumbbell-Halbach by numerical simulations, and reached improvement from $26 \mathrm{ppm}$ to $0.5 \mathrm{ppm}$ over a length of $3 \mathrm{~mm}$, which was beyond our measurement capability of 3 ppm.
\end{abstract}

\subsection{Introduction}

Mobile NMR-sensors are frequently used in chemistry or biology. The main characteristics of those type of sensors are low-cost, low-energy consumption, lightweight and relatively small dimensions. Mobile NMR sensors are often based on a Halbach dipole magnet, a configuration that has firstly been introduced by Klaus Halbach (Halbach, 1985). Halbach-ring permanent magnet arrangements enhance the field in the bore, reaching up to $2 \mathrm{~T}$ while reducing the stray-field

This chapter is based on Y.P.Klein, L. Abelmann, J.G.E.Gardeniers, "Dumbbell Pseudo Halbach Magnet Configuration: Combining high field strength with high homogeneity" to be submitted to J. Magn. Reson. 
(Tayler and Sakellariou, 2017). Combining such a configuration with long magnets results in a high field along the entire bore, which is especially beneficial for mobile microfluidic flow measurements where the solenoid coil is wound around a thin channel in which the sample-fluid is located. Therefore Halbach-magnets are usually rather long, ranging from $70 \mathrm{~mm}$ to $240 \mathrm{~mm}$ with a weight of a few $\mathrm{kg}$. Still, the homogeneity without electric shimming does not reach the homogeneity needed for high resolution NMR measurements, typically falls in a range between 20 and 2300 ppm (Hills et al., 2005; Hua et al., 2017; Moresi and Magin, 2003; Vogel et al., 2016; Windt et al., 2011). The homogeneity of Halbach configurations can be improved with the combination of multiple Mandala rings where ring distance has been tuned to improve the field homogeneity (Hugon et al., 2010; Sakellariou et al., 2010; Soltner and Blümler, 2010). A drawback of using a gap between the rings is the lower resulting field strength. We present a configuration which combines a high magnetic field arising from the Pseudo-Halbach configuration and the possibility to shim the field easily mechanically with additional magnetic rings.

The field-homogeneity gets further improved with the use of electric shimming units(Golay, 1958), where an arbitrary shimming field is superposed out of spatial harmonics (Anderson, 1961; Roméo and Hoult, 1984), which are generated by multiple shim coils. Those shimming units come in several shapes, but especially for small mobile NMR sensors, the planar electric shimming elements are of high interest. In particular using biplanar shimming coils is a well-known approach to achieve better homogeneities (Crozier et al., 1995; Liu et al., 2007; Martens et al., 1991; You et al., 2010). Tamada et al. (Tamada et al., 2012) presented a planar shimming chip which is based on multiple circular current rings next to each other. Another interesting concept was presented by Van Meerten et al. who applied a small shimming chip, specially developed for thin capillary tubes (van Meerten et al., 2018). This new chip consists out of multiple parallel planar wires which can be driven separately to achieve several shimming field profiles along the channel. In this work we investigate the performance of our new magnet-configuration in combination with a simple electric shimming unit.

\subsection{Methods}

\subsubsection{Field calculations}

To study the magnetic field pattern and to improve the field-homogeneity, we simulated the Pseudo-Halbach magnet configuration in combination with mechanical shimming rings and electrical shimming circuits. The simulations have been done using CADES simulation software, described by Delinchant et al. (Delinchant et al., 2007). Magnetic interactions are modeled with the MacMMems tool, which uses the Coulombian equivalent (for permanent magnets), 


$$
\begin{array}{r}
\mathbf{H}(\mathbf{r})=\iint_{\mathrm{S}} \frac{\sigma_{\mathrm{S}} \cdot\left(\mathbf{r}-\mathbf{r}^{\prime}\right)}{\left|\mathbf{r}-\mathbf{r}^{\prime}\right|^{3}} d s+\iiint_{V} \frac{\rho_{\mathrm{V}} \cdot\left(\mathbf{r}-\mathbf{r}^{\prime}\right)}{\left|\mathbf{r}-\mathbf{r}^{\prime}\right|^{3}} d v \\
\sigma_{\mathrm{S}}=\mathbf{M} \cdot \mathbf{n}, \rho_{\mathrm{V}}=-\operatorname{div}(\mathbf{M})
\end{array}
$$

and Biot and Savart (for conductors) to generate a semi-analytic model.

$$
\mathbf{H}(\mathbf{r})=\frac{1}{4 \pi} \iiint_{\mathrm{V}} \frac{\mathbf{j} \cdot\left(\mathbf{r}-\mathbf{r}^{\prime}\right)}{\left|\mathbf{r}-\mathbf{r}^{\prime}\right|^{3}} d v
$$

Here, $\mathbf{H}$ is the magnetic field intensity and $\mathbf{M}$ the magnetization of the permanent magnet (both in $\mathrm{A} / \mathrm{m}$ ), $\mathbf{j}$ as the current density (in $\mathrm{A} / \mathrm{m}^{2}$ ), $\mathbf{r}$ and $\mathbf{r}^{\prime}$ define the observation point and its distance to the elementary field source enclosed by $d s$ and $d \nu, \sigma_{\mathrm{S}}$ and $\rho_{\mathrm{V}}$ are the surface and volume charge, and $\mathbf{n}$ the unit vector normal to the surface. with $\mathbf{j}$ as the current density. The generated analytical expressions are used by the CADES framework (component generator, component calculator, component optimizer) to calculate and optimize the design.

\subsubsection{Experimental}

The magnets, in our case with an edge length $d=7 \mathrm{~mm}$, have been assembled with a magnet holder made from aluminum. To minimize the temperature-drift, we chose to use $\mathrm{Sm}_{2} \mathrm{Co} 17$ ( $\left.\mathrm{H}_{2} 6\right)$ (purchased from Schallemkammer Magnetsysteme $\mathrm{GmbH})$ with a temperature coefficient of $-0.035 \%{ }^{\circ} \mathrm{C}^{-1}$. The total size of the Dumbbell-configuration including aluminum holder is $50 \times 50 \times 42 \mathrm{~mm}^{3}$ with a weight of $332 \mathrm{~g}$. A photograph of the assembled magnet system is shown in Fig. 3.1. PCBs with the shimming elements shown in Fig. 3.2 (4 layers, $0.035 \mathrm{~mm}$ copper thickness) have been ordered from Würth Elektronik GmbH \& Co. KG. Each layer (blue, red) are 2 times present in a single chip.

The measurement setup is shown in Fig. 3.3. A Hall sensor from Projekt Elektronik GmbH (Teslameter 3002/Transverse Probe T3-1,4-5,0-70, sensitive area $(1.5 \mathrm{~mm} \times 3.0 \mathrm{~mm})$ in combination with a motorized linear stage has been used to measure the field of magnet configuration along the bore in a temperature stable environment $\left( \pm 0.5^{\circ} \mathrm{C}\right)$. Since the sensor is in a fixed horizontal position and just the magnet has been moved exactly horizontal that field-variations caused by the setup have no influence on the measurement. 
Chapter 3-Dumbbell Pseudo Halbach Magnet Configuration: Combining high 36 field strength with high homogeneity

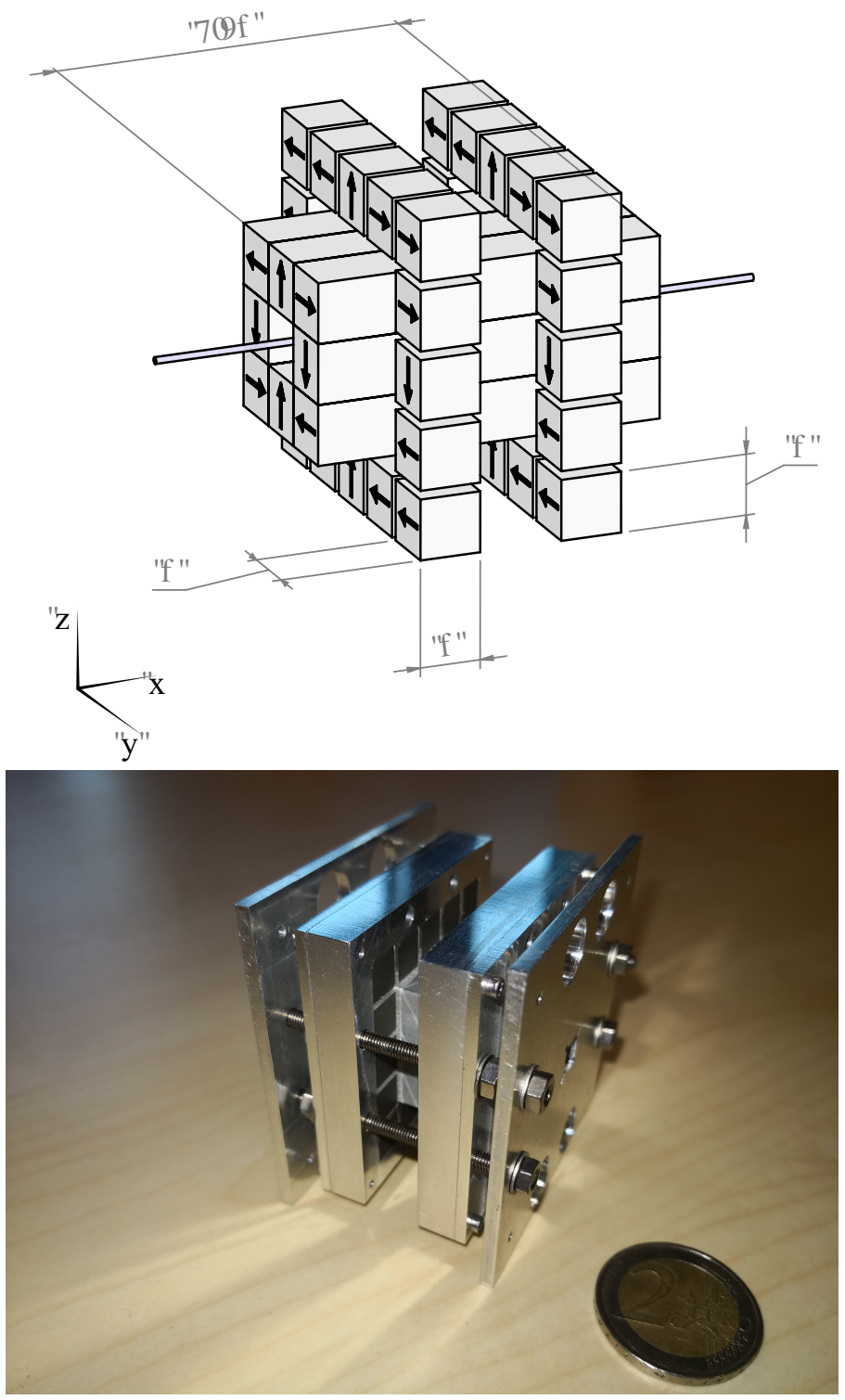

FIGURE 3.1 - Top:Schematic view of the Dumbbell-Halbach configuration which is a combination of the Pseudo-Halbach consisting out of 8 bar magnets and 2 movable shimming rings, each one consists out of 16 cubic magnets. The capillary tube in the bore contains the sample liquid. Bottom: Implementation of the Dumbbell-Halbach magnet 


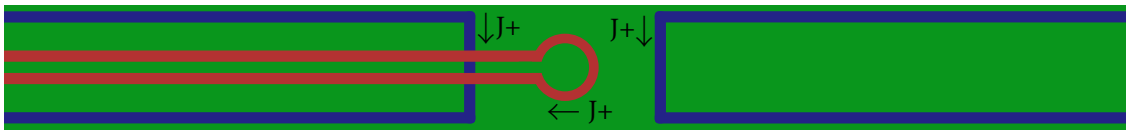

FIGURE 3.2 - Layout of the experimental shimming circuit, the PCB (green) contains 2 striplines (blue, center-distance, $2 \mathrm{~mm}$, distance feed-line: $4 \mathrm{~mm}$ ) and 1 ring (red, inner radius: $1 \mathrm{~mm}$, distance feed-line: $500 \mu \mathrm{m}$ ). Conductor path: width: $500 \mu \mathrm{m}$, height: $105 \mu \mathrm{m}$.

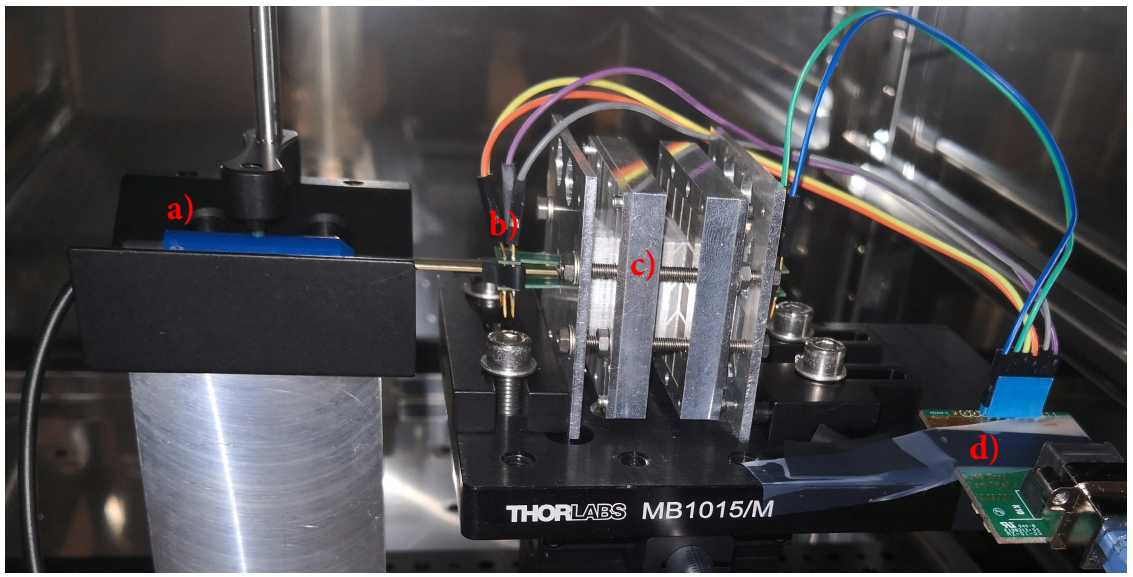

FIGURE 3.3-Setup for the measurement of the magnetic field profile in the Dumbbell-Halbach magnet (c), using a Hall sensor (a). The wiring and $P C B(d)$ are used to drive the shimming coils $(b)$. The Hall sensor is fixed in the position in middle of the bore of the magnet, whereas the magnet is moved horizontally with a positioning stage.

\subsection{Results and Discussion}

We propose a design based on the Halbach configuration. The residual nonuniformity in the field is compensated first by a mechanical shimming approach using movable rings. The remaining non-uniformity is reduced further by current conductors.

\subsubsection{Mechanical shimming: Dumbbell-Halbach}

Since the radius of the sample-containing channel is small, we will ignore field variations perpendicular to the channel ( $y$ - and $z$-direction) (Fig.3.13 in appendix). Fig. 3.4 shows in black the variation in the normalized field of the Pseudo-Hallbach assembly along the length of the bore of the Pseudo-Halbach, normalized to the 


\section{Chapter 3 - Dumbbell Pseudo Halbach Magnet Configuration: Combining high 38 \\ field strength with high homogeneity}

magnet dimension $d$ (defined in Fig. 3.1). The field profile is parabolic with a maximum of $0.91 B_{\mathrm{s}}$ in the center, dropping towards the magnet edges. The variation in the field strength over a length $d$ along the bore, normalized to the field in the center, is $1793 \mathrm{ppm}$.

A complementary field can be generated by two shimming magnet rings, as shown by the red curve in figure 3.4. The distance between the rings can be adjusted to reach minimal variation in the $z$-component of the sum of both fields.

This mechanical shimming strategy is applied to a novel configuration, which we nicknamed the Dumbbell-Halbach. It consists of 8 bar magnets $(d \times d \times$ 5.7d) arranged as a Pseudo-Halbach with an inner distance of $d$ (Fig. 3.1). The magnetization of each bar magnet is chosen in such a way, that the field in the bore is enhanced while reducing the stray-field (see Fig. 3.12 in the Appendix). Two adjustable shimming rings, consisting of 16 cubic magnets each with an edge length of $d$, are slid over the Pseudo-Halbach. For practical assembly reasons, the magnets have a distance of $1 / 7 d$ to each other and a distance of $2 / 7 d$ from the Pseudo-Halbach (see Fig. 3.1).

The field profile of the novel Dumbbell-Halbach configuration is shown in Fig. 3.5. The normalized difference of the field-maxima and minima has dropped to $297 \mathrm{ppm}$, which is an improvement by a factor of six with the Pseudo-Halbach design. The field in the center reaches $1.08 B_{\mathrm{s}}$, which is an improvement of $19 \%$.

The distance between the shimming rings can be adjusted to accommodate for different sample lengths. Fig. 3.6 shows the optimal ring distance (blue) and achievable homogeneity (black) as a function of the desired sample length. Decreasing the sample length results in a better total homogeneity along the bore. For a sample-length of $0.5 d$ the optimum ring-distance is $0.97 d$ and a homogeneity of $8 \mathrm{ppm}$ can be reached. For twice the sample length $(d)$, the ring distance needs to be increased to $1.04 d$, but the homogeneity deteriorates by more than a factor of ten $(104 \mathrm{ppm})$. To counteract against magnet-variations in reality, we recommend small mechanical displacements of each ring after following the described shimming-procedure.

Fig. 3.7 shows the improvement of the field homogeneity when moving from the Pseudo-Halbach to the Dumbbell-Halbach. The shimming rings are most effective at small sample lengths, improving the homogeneity 16.5 times for a sample length of $0.5 d$ length, down to 5 times for a sample length of $d$.

\subsubsection{Electric shimming}

In the calculations presented above, we assume that all magnets in the configuration are identical. In reality, magnets will show variation in both magnetization strength and orientation. Typical variations are around $1 \%$ and $1{ }^{\circ}$, respectively (chapter 2 ). These magnet variations will lead to an small deviations from the ideal magnetic field gradient. Since the deviations are small, we suggest to compensate them electrically by shimming striplines.

The variations from the ideal situation can be both symmetric and antisymmetric with respect to the sample center $(x=0)$. Therefore we employ 


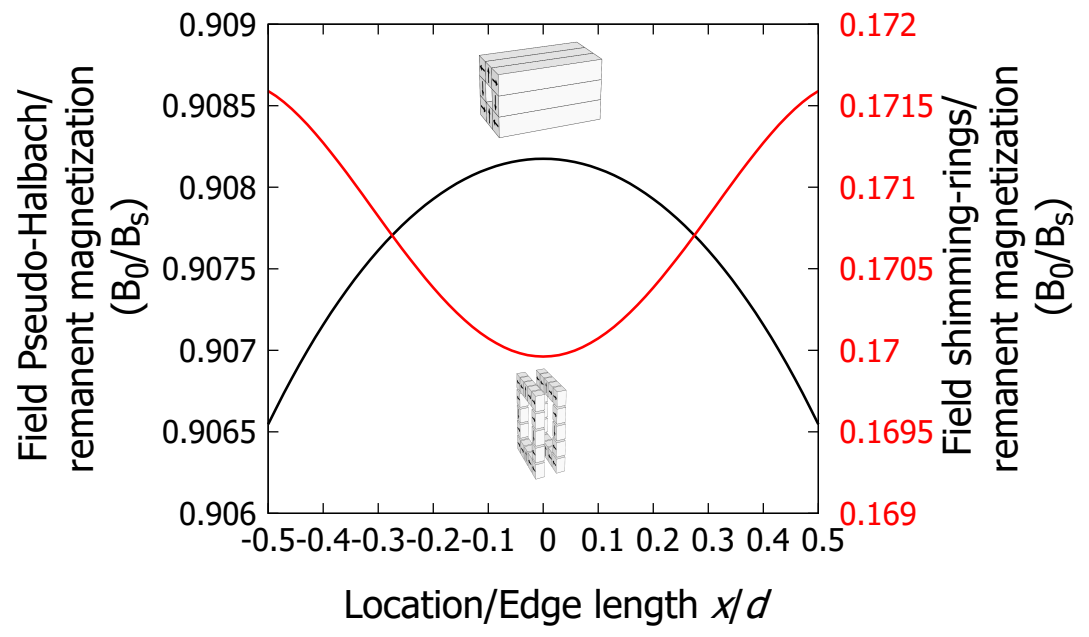

FIgURE 3.4 - Magnetic field of the Pseudo-Halbach (black) and the separate shimming magnet rings (red) along the normalized bore length of the Halbach magnet. The Pseudo-Halbach alone shows a parabolic field profile with its maximum in the center. The rings generate a complementary field with a minimum in the center.

two shimming circuits, as illustrated in Fig. 3.8. The circuit combines two planar wires (blue) used to generate a anti-symmetric linear gradient along the bore and a ring (red) to generate a symmetric parabolic field. The distance of the planar wires to each other $(4 \mathrm{~mm})$ and the radius of the ring $(1 \mathrm{~mm})$ have been optimized for our Dumbbell-Halbach using the CADES optimizer program. To minimize the influence of the electrical feed-line for the ring, the wires have been chosen to be close to each other. Each shimming-element can be driven separately.

The ring itself generates a parabolic field profile (Fig. 3.9, red curve), while the striplines alone result in a linear gradient (blue curve). A combination of a separately driven ring and striplines (red curve) provide the possibility to shim the main valley in the magnetic field profile and to add a linear gradient, considering that it is quite unlikely that the mechanic shimming rings have exactly the same magnetization. Compared with the parabolic field produced by striplines, the rings alone reach a two times higher peak field strength.

The electric shimming ring not only corrects for permanent magnet variation, but also improves the residual inhomogeneity that is left by the magnetic shimming rings of the Dumbbell-Halbach configuration. Calculations show (Fig. 3.14 in 


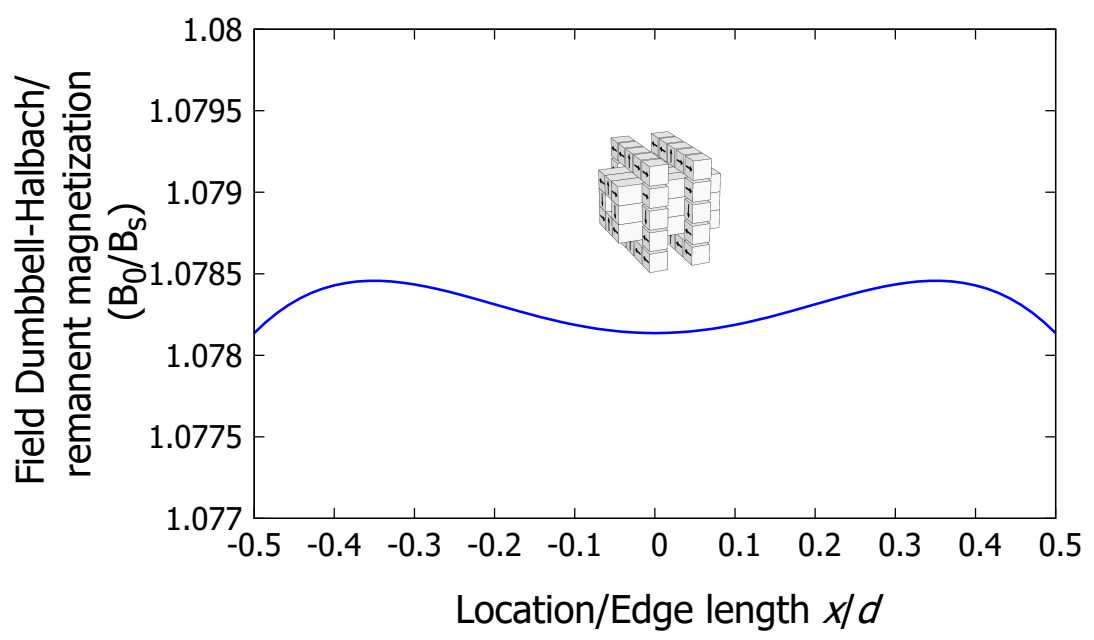

FIGURE 3.5 - The maximal field strength of the Dumbbell-Halbach is $1.0785 B_{\text {mean }}$. The field shows a more homogeneous profile than the Pseudo-Halbach without shimming rings as shown in Fig.3.4.

appendix) that the shimming ring improves the homogeneity along the $x$-axis for a sample length of $3 \mathrm{~mm}$ from $26 \mathrm{ppm}$ down to $0.5 \mathrm{ppm}$ (ring radius of $1 \mathrm{~mm}$ driven with a current density of $0.9345 \mathrm{~A} / \mathrm{mm}^{2}$ ).

\subsubsection{Experimental validation}

To confirm the predictions of our simulations, the Dumbbell-Halbach configuration was assembled and field profiles were measured. We considered three different sample sizes. We measured a homogeneity of $33 \mathrm{ppm}$ over a length of $5 \mathrm{~mm}$, $381 \mathrm{ppm}$ over a length of $10 \mathrm{~mm}$ and $1862 \mathrm{ppm}$ over a length of $16 \mathrm{~mm}$. The measurements differ by $5 \%$ from the simulation results for the $16 \mathrm{~mm}$ sample up to $15 \%$ for the $5 \mathrm{~mm}$ sample (Table 3.1)). The mismatch between experiment and simulation can be explained by misalignment of the Hall-probe or magnetization and magnetization angle variations between the magnets.

We measured the field of each channel of our biplanar electric shimming circuit whilst mounted in the permanent magnet system, by subtracting the unshimmed field. This also eliminates background fields, such as the earth magnetic field. For this experiment we used a current density of $4.5 \mathrm{~A} / \mathrm{mm}^{2}$ for the rings and $2.7 \mathrm{~A} / \mathrm{mm}^{2}$ for the stripline located on the negative $x$-axis and a current density of 


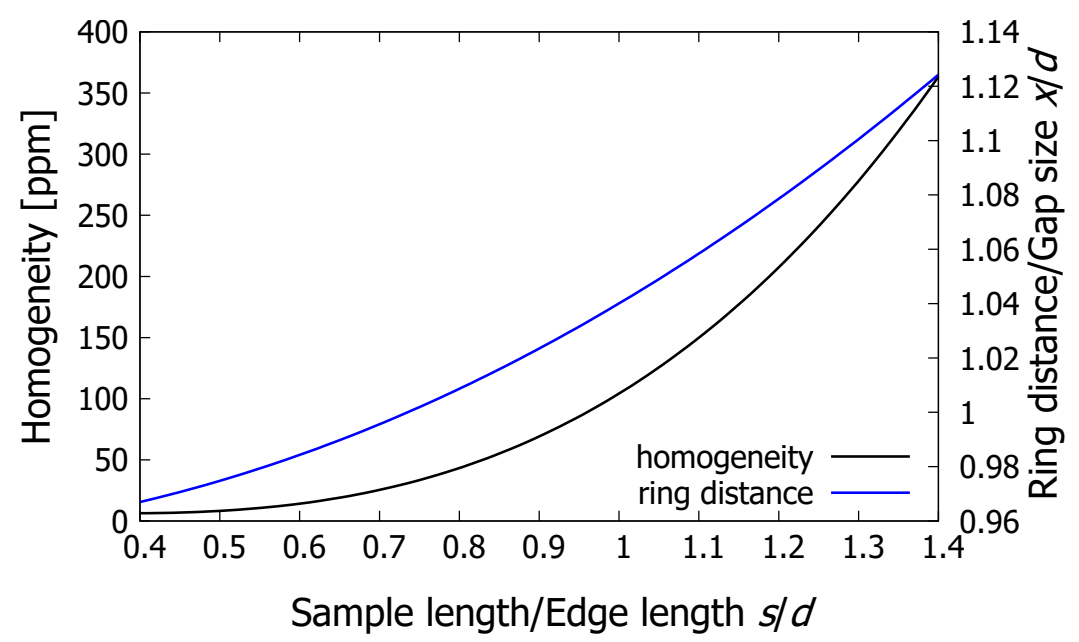

FIGURE 3.6 - Field homogeneity and ring distance as a function of the sample length. The closer the rings are positioned together, the higher the field homogeneity.

TABLE 3.1 - Comparison of simulated and measured homogeneities. For each sample length the rings have been adjusted to reach the minimal possible homogeneity. Over a range of $5 \mathrm{~mm}$, a homogeneity of 33 ppm has been measured, $15 \%$ larger than the simulated value. The measurements for the higher ranges diverge less. The simulated homogeneity for $10 \mathrm{~mm}$ is $3 \%$ worse, for $16 \mathrm{~mm} 5 \%$. The shorter the measurement range, the higher the maximal field strength, caused by the shorter distance of the shimming rings. The measurement accuracy of the used Hall-probe is 3 ppm.

\begin{tabular}{lccc}
\hline \hline Sample length [mm] & 5 & 10 & 16 \\
\hline Simulated homogeneity [ppm] & 28 & 391 & 1958 \\
Measured homogeneity $[\mathrm{ppm}]$ & $33(3)$ & $381(3)$ & $1862(3)$ \\
Simulated max. field strength $\left[B_{\mathrm{O}} / B_{\mathrm{S}}\right]$ & 1.081 & 1.073 & 1.059 \\
Measured max. field strength $[\mathrm{T}]$ & 1.058 & 1.049 & 1.031 \\
\hline \hline
\end{tabular}


Chapter 3-Dumbbell Pseudo Halbach Magnet Configuration: Combining high 42

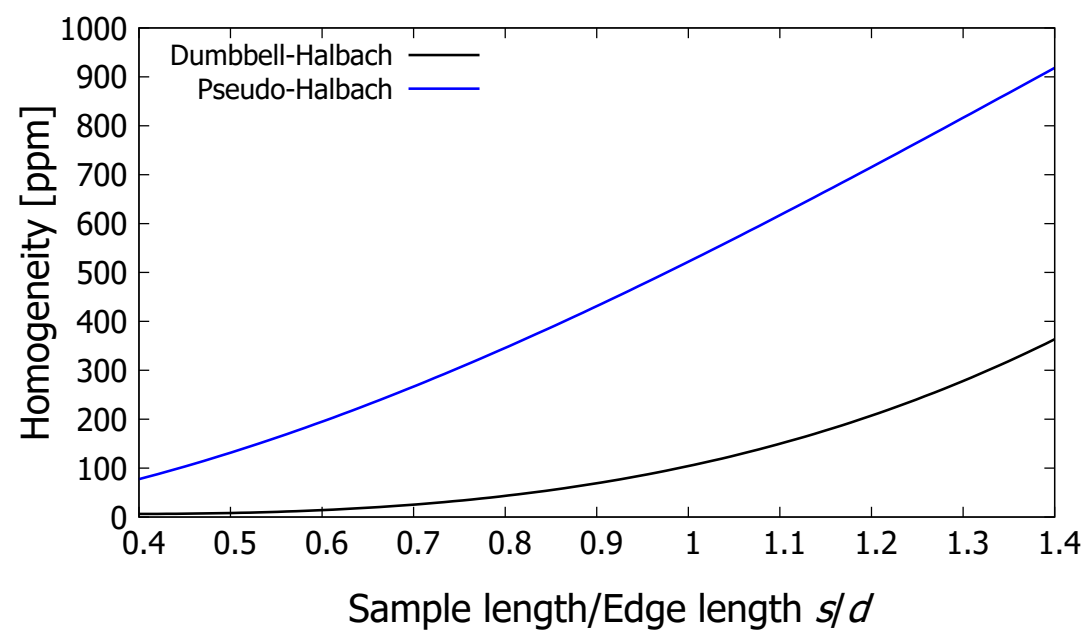

FIGURE 3.7 - The improvement of homogeneity due to addition of the shimming rings depends on the sample length. In general, homogeneity improves with reducing sample length. The Pseudo-Halbach reaches 132 ppm for a sample-length of 0.5 d, whereas the Dumbbell-Halbach reaches a homogeneity of 8 ppm. At a sample length of $d$, the difference decreases to a factor of five $(522$ versus 104 ppm).

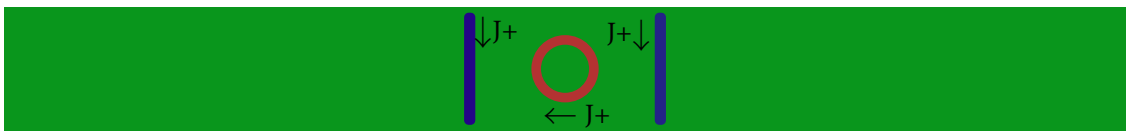

Figure 3.8 - Layout of the simulated shimming PCB (green), consisting out of 2 striplines (blue, center-distance, $2 \mathrm{~mm}$ ) and 1 ring (red, inner radius: $1 \mathrm{~mm}$ ). Conductor path width $500 \mathrm{\mu m}$, height: $105 \mathrm{mu} \mathrm{m}, \mathrm{J}: 0.0525 \mathrm{~A} / \mathrm{mm}^{2}$ ). 


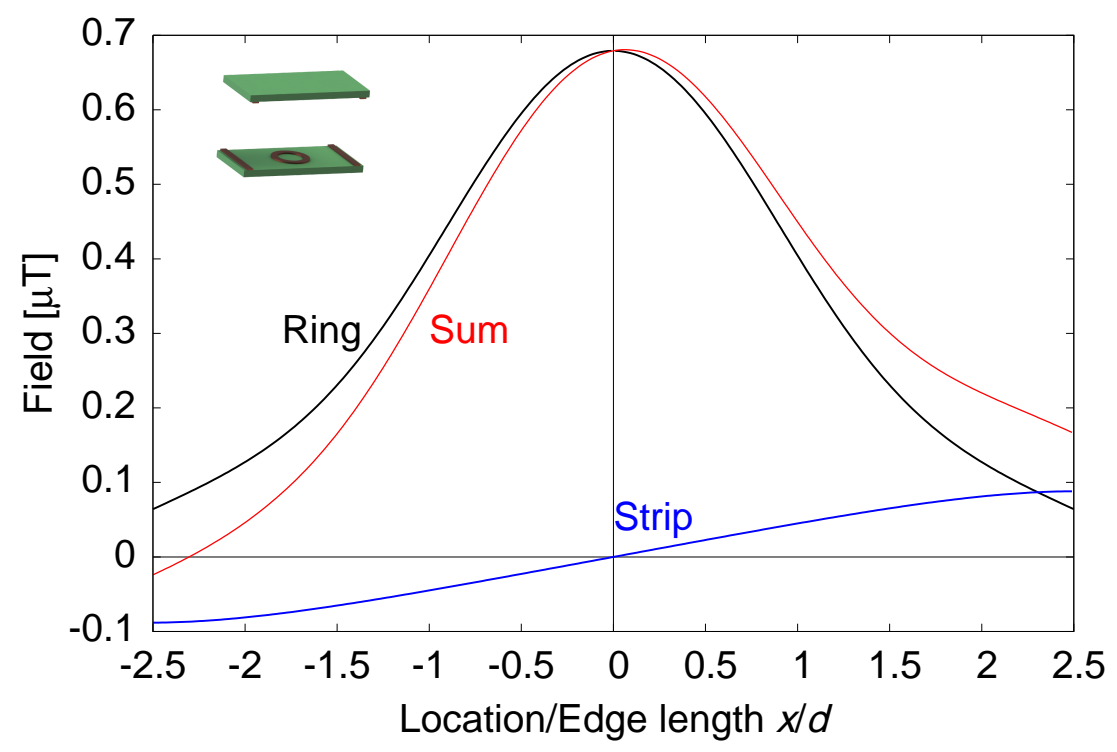

FIGURE 3.9 - Field profile caused by chip-sandwich (distance $5 \mathrm{~mm}$ ). The resulting field (red curve) is a combination of the field of the two driven striplines with anti-parallel current at distance $4 \mathrm{~mm}$ (blue curve) and the center ring with inner radius $1 \mathrm{~mm}$ (black curve). Conductor path width: $500 \mu \mathrm{m}$, height: $105 \mu$ m, J: $0.0525 \mathrm{~A} / \mathrm{mm}^{2}$ )

$2.5 \mathrm{~A} / \mathrm{mm}^{2}$ for the stripline located on the positive $x$-axis.

Fig. 3.9 shows the total magnetic stray field generated by the strip lines (red and blue curves). Since the stripline on the negative $x$-axis (blue) has a slightly higher current, its field is higher, causing the asymmetry around $x=0$ between the left and right stripline. The shimming ring (black curve) reaches a maximum of $35 \mu \mathrm{T}$ at $0 \mathrm{~mm}$, dropping to $24 \mu \mathrm{T}$ at $x=-2 \mathrm{~mm}$ and to $2 \mu \mathrm{T}$ at $x=3 \mathrm{~mm}$. The slight asymmetry in the field might be caused by wiring leading the current into the ring.

Electrical shimming with our shimming chip pushes the homogeneity to values beyond the accuracy of our Hall sensor. Fig. 3.10 shows the $z$-component of the field of the Dumbbell-Halbach as a function of the position along the channel (black curve). The field profile shows a field difference the maximal and minimal value of $60 \mu \mathrm{T}$ while the difference between the two peaks is $11 \mu \mathrm{T}$. So by mechanical shimming only we can reach a homogeneity of $23 \mathrm{ppm}$ over a distance of $3.5 \mathrm{~mm}$.

Using the CADES optimizer routines, the measured profile was used to obtain the current densities for the shimming chip, resulting in current densities of $\mathrm{X}$ : $6.375 \mathrm{~A} / \mathrm{m}^{2}, \mathrm{Y}: 9 \mathrm{~A} / \mathrm{m}^{2}, \mathrm{Z}: 7.29 \mathrm{~A} / \mathrm{m}^{2}$. The blue measurement points in figure 3.11 


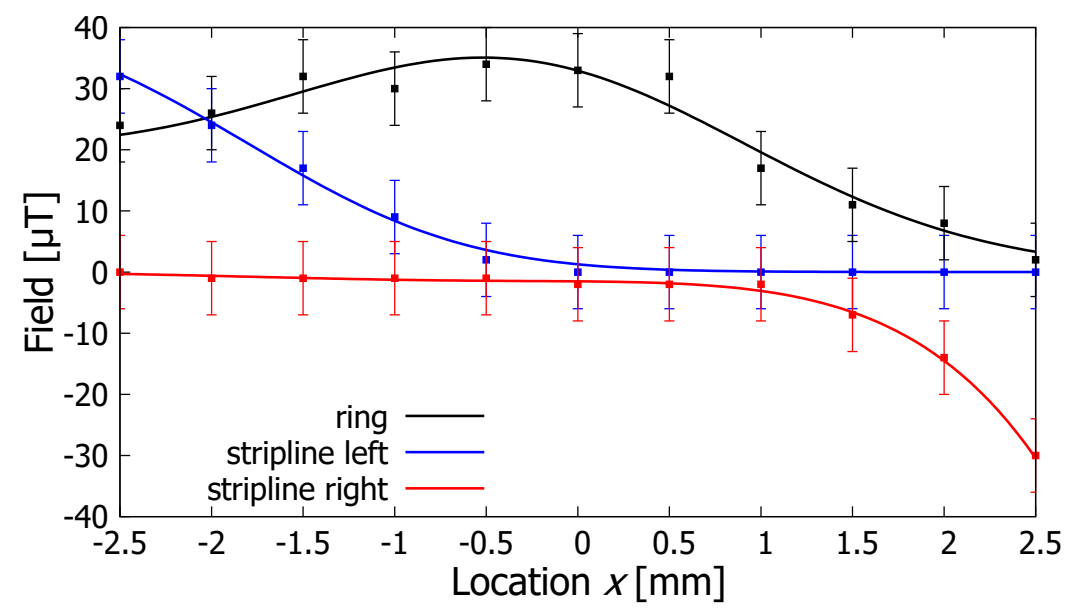

FIGURE 3.10 - Measured shimming field produced by the chip-sandwich. Each stripline generates a linear field profile (blue, red), the ring a parabolic one (black). The solid line shows the fitting function for each profile.

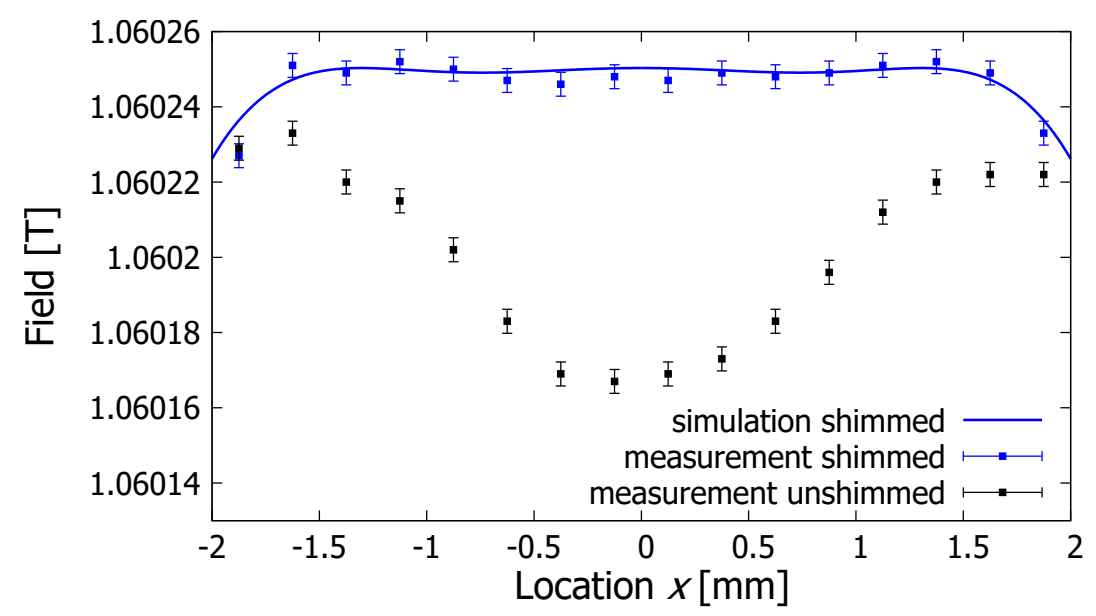

FIGURE 3.11 - Measurement results: Homogeneity before shimming (black): 23(3) ppm, homogeneity after shimming (blue): 2(3) ppm. 
show the resulting profile with electrical shimming, which is in agreement with our prediction with the error of our Hall probe $(2 \mathrm{ppm}+-3 \mathrm{ppm})$ over a length of $3.5 \mathrm{~mm}$.

\subsection{Conclusions}

An improved Pseudo-Halbach has been invented for which the homogeneity can be tuned with additional shimming rings depending on the length of interest within the bore. The magnet configuration has been build using $\operatorname{Sm} 2 \mathrm{Co17}(26 \mathrm{H})$ magnets and an aluminum frame with a total size of $50 \mathrm{~mm} \times 50 \mathrm{~mm} \times 42 \mathrm{~mm}$ and a weight of $332 \mathrm{~g}$. The field-strength of the Dumbbell-Halbach is $19 \%$ higher compared to the Pseudo-Halbach without rings, the homogeneity can be improved $1650 \%$ regarding a sample length of 0.5 edge length of the magnet cube. Measurements have shown a homogeneity of $33 \mathrm{ppm}$ and $1.06 \mathrm{~T}$ for a sample length of $5 \mathrm{~mm}$ without electric shimming which is in agreement with simulations within an error of $10 \%$. We simulated an improvement from $26 \mathrm{ppm}$ to $0.5 \mathrm{ppm}$ over a length of $3.5 \mathrm{~mm}$ using electric shimming chips. We confirmed that indeed the field homogeneity was improved beyond the measurement capability of our Hall-probe (3 ppm).

\subsection{Appendix}

A small radius of the sample-containing channel can be assumed if the $z$-field difference along the $y$ - and $z$-axis is low enough. To keep the characteristic Halbach

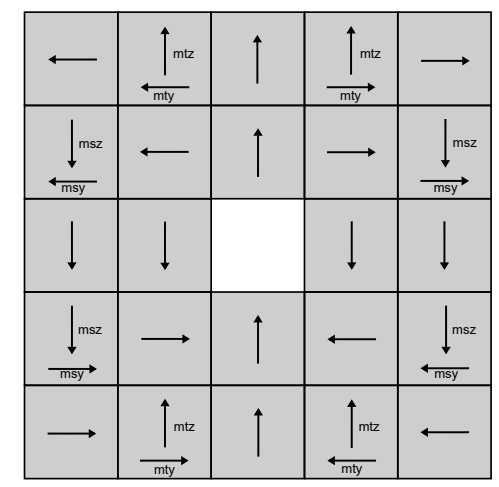

FIGURE 3.12 - Variations of magnetization direction for the used shimming rings. 


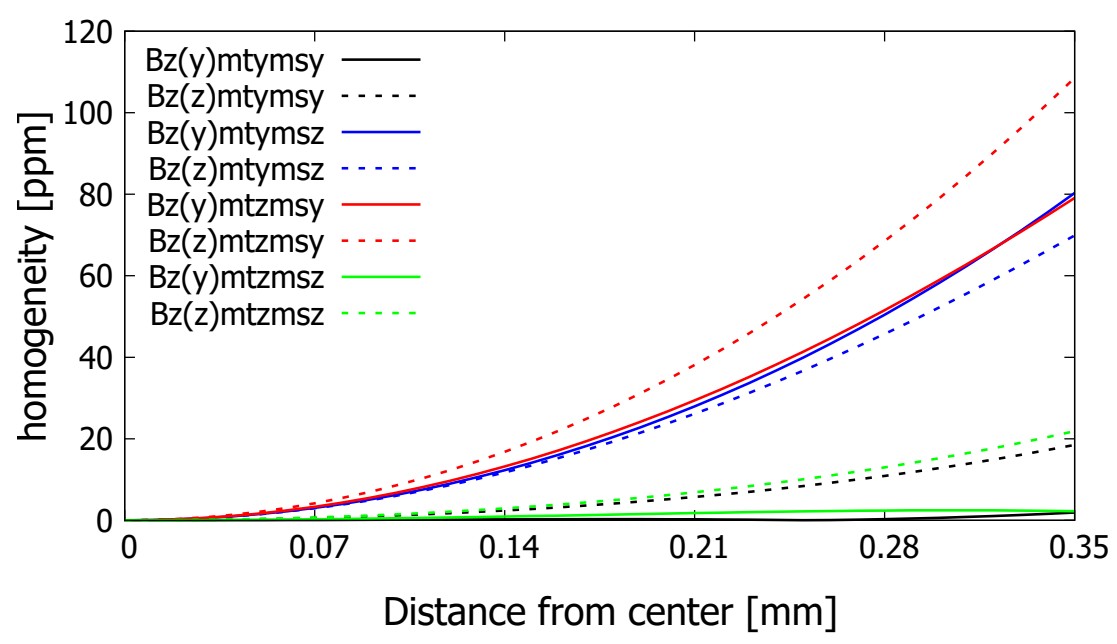

FIGURE 3.13-Field difference of the Dumbbell-Halbach configuration from the center in y and $z$ direction. Configuration "mtymsy" shows the lowest field difference.

field behavior intact, the middle and corner magnetization direction of the rings is the same as the inner Pseudo-Halbach. The magnets in between have been varied as shown in Fig. 3.12. The direction of magnetization is named as following: mtz stands for magnetization of the top and bottom magnets in $\mathbf{z}$-direction. msy stands for magnetization of the side magnets in $\mathbf{y}$-direction.

Fig. 3.14 shows the $z$-field difference along the y-axis (straight line) and $z$-axis (dashed line) from the center of the Dumbbell-configuration. For each parameter-set, the ring-distance have optimized for a sample length of $8 / 7 d$. 


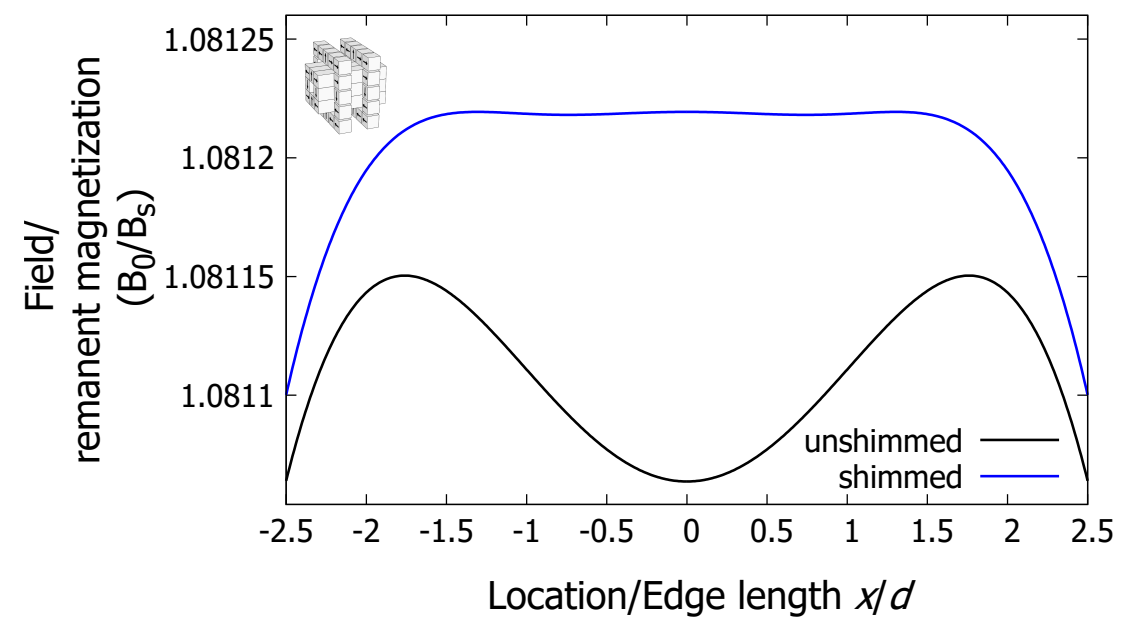

FIGURE 3.14 - Simulation: Homogeneity before shimming (black): 26 ppm, homogeneity after shimming (blue): $0.5 \mathrm{ppm}$

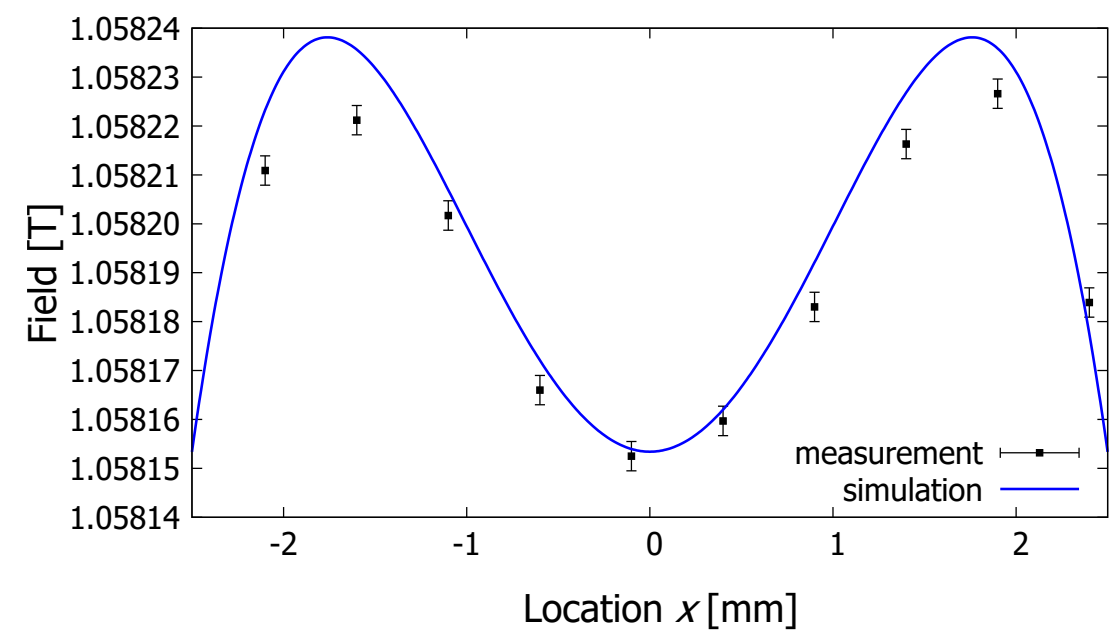

FIGURE 3.15 - Measurement results: Ring distance optimized for a sample length of $5 \mathrm{~mm}$. 
Chapter 3 - Dumbbell Pseudo Halbach Magnet Configuration: Combining high 48

field strength with high homogeneity

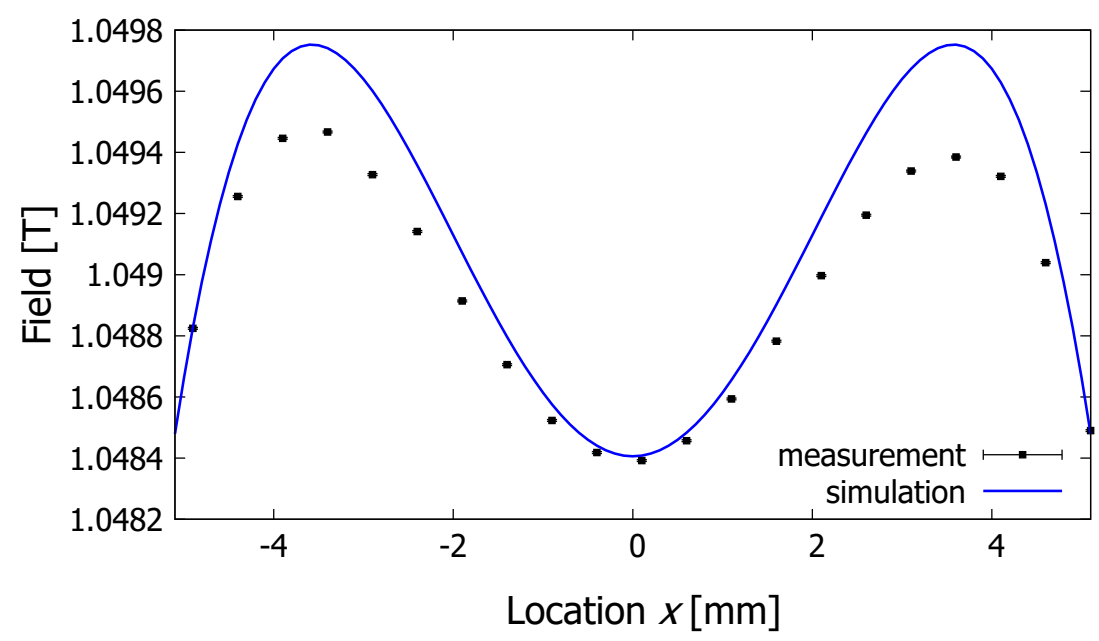

FIGURE 3.16 - Measurement results: Ring distance optimized for a sample length of $10 \mathrm{~mm}$.

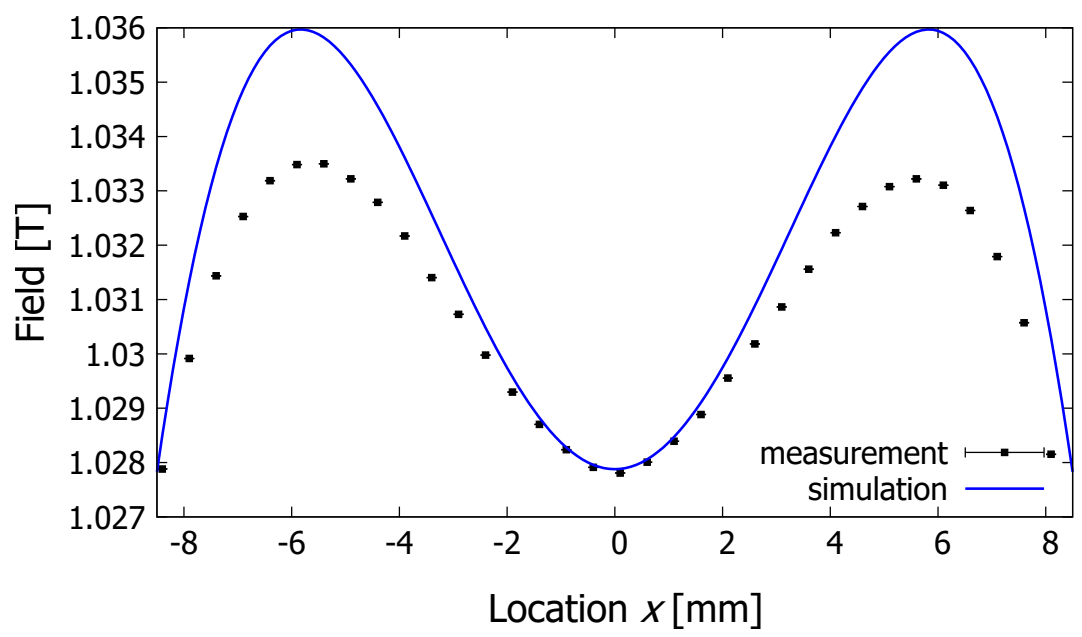

FIGURE 3.17 - Measurement results: Ring distance optimized for a sample length of $16 \mathrm{~mm}$. 


\title{
Chapter 4
}

\section{Shim on a chip on small scale using ferrofluids}

\begin{abstract}
Mobile NMR (Nuclear Magnetic Resonance) systems require a high magnetic field-homogeneity which usually is optimized with the help of either mechanic or electric shimming units. In this paper we present a novel possibility to further improve the field-homogeneity of miniaturized magnet systems with the help of ferrofluid containing microfluidic-chips. We demonstrated their use with two different options to change the magnetic moment. With both, i.e. changing the volume or changing the saturation magnetization, we prove the feasibility of applying ferrofluids in a permanent magnet system to improve the homogeneity. Changing the volume resulted in a 4 times better homogeneity over a range of $11.0 \mathrm{~mm}$ (434 ppm to $111 \mathrm{ppm}$ ), alternating the saturation magnetization of the ferrofluid could improve the homogeneity from $86 \mathrm{ppm}$ to $8 \mathrm{ppm}$ over a length of $5.5 \mathrm{~mm}$.
\end{abstract}

\subsection{Introduction}

Downscaling permanent magnet configurations is an always continuing trend for mobile NMR-systems. However their magnetization and fabrication variations constitute problems when it comes to reach field-homogeneities which are needed for high-resolution NMR. A common way to improve the field homogeneity of magnet configurations is electric shimming, where solenoid coils, planar coils or striplines are generating a field to counteract against the inhomogeneities of the magnets (Liu et al., 2007; van Meerten et al., 2018; Tamada et al., 2012). This technique is energy consuming and resistance issues will finally limit use of electric shimming chips in extremely small magnet systems. In order to be able to shim the field in miniaturized permanent magnet systems, we present a new

This chapter is based on Y.P.Klein, L. Abelmann, J.G.E.Gardeniers, "Shim on a chip on small scale using ferrofluids" to be submitted to J. Lab on a Chip 
approach: Ferrofluids in combination with microfluidic chips. Microfluidic chips have the advantage that containers and cavities can be fabricated with accuracies in the range of $\mu \mathrm{m}$ or better (de Boer et al., 2000).

Ferrofluids have firstly been synthesized by Papell in 1964 (Papell, 1964) and cover nowadays a broad spectrum of applications (B.M. Berkovsky, 1996). The usage ranges from sealing (Pinho et al., 2014; Volder and Reynaerts, 2009), material separation (Zeng et al., 2013), medicine (Alexiou et al., 2001; Hergt et al., 1998) to sensors (Ahmed et al., 2019) and robotics (Yu et al., 2020) to name a few examples. Aim of this research is to characterize how ferrofluids can be used to tune and improve the field-homogeneity of permanent magnet-systems. We will start with computational modeling of microfluidic elements filled with ferrofluids, to get an idea about the range of magnetic fields that can be obtained. Furthermore, to demonstrate the feasibility, we have fabricated microfluidic chips with different volumes of ferrofluids, and ferrofluids with different magnetization levels, and tested them in a special type of Halbach magnet that was described in the previous chapter.

\subsection{Methods}

\subsubsection{Computational method}

The simulations have been done using CADES simulation software, completely described by Delinchant et al. (Delinchant et al., 2007). The magnetic interactions are modelled with the MacMMems tool, which uses the Coulombian equivalent charge method to generate a semi-analytic model.

$$
\begin{array}{r}
\mathbf{H}(\mathbf{r})=\iint_{S} \frac{\sigma_{S} \cdot\left(\mathbf{r}-\mathbf{r}^{\prime}\right)}{\left|\mathbf{r}-\mathbf{r}^{\prime}\right|^{3}} d s+\iiint_{V} \frac{\rho_{V} \cdot\left(\mathbf{r}-\mathbf{r}^{\prime}\right)}{\left|\mathbf{r}-\mathbf{r}^{\prime}\right|^{3}} d v \\
\sigma_{S}=\mathbf{M} \cdot \mathbf{n}, \rho_{V}=-\operatorname{div}(\mathbf{M})
\end{array}
$$

Here, $\mathbf{H}$ is the magnetic field intensity and $\mathbf{M}$ the magnetisation of the permanent magnet (both in $\mathrm{A} / \mathrm{m}$ ), $\mathbf{r}$ and $\mathbf{r}^{\prime}$ define the observation point and its distance to the elementary field source enclosed by $d s$ and $d \nu, \sigma_{S}$ and $\rho_{V}$ are the surface and volume charge, and $\mathbf{n}$ the unit vector normal to the surface.

The CADES framework, including a component generator, component calculator, and component optimizer, generated the final equations, which are used to calculate and optimize the designs.

\subsubsection{Dumbbell-Halbach}

The magnet design we used in our experimental setup is shown in Fig. 4.1. One key benefit of this magnet configuration is the possibility to tune the magnetic field depending on the sample length. The complete configuration is described in chapter 3 . 


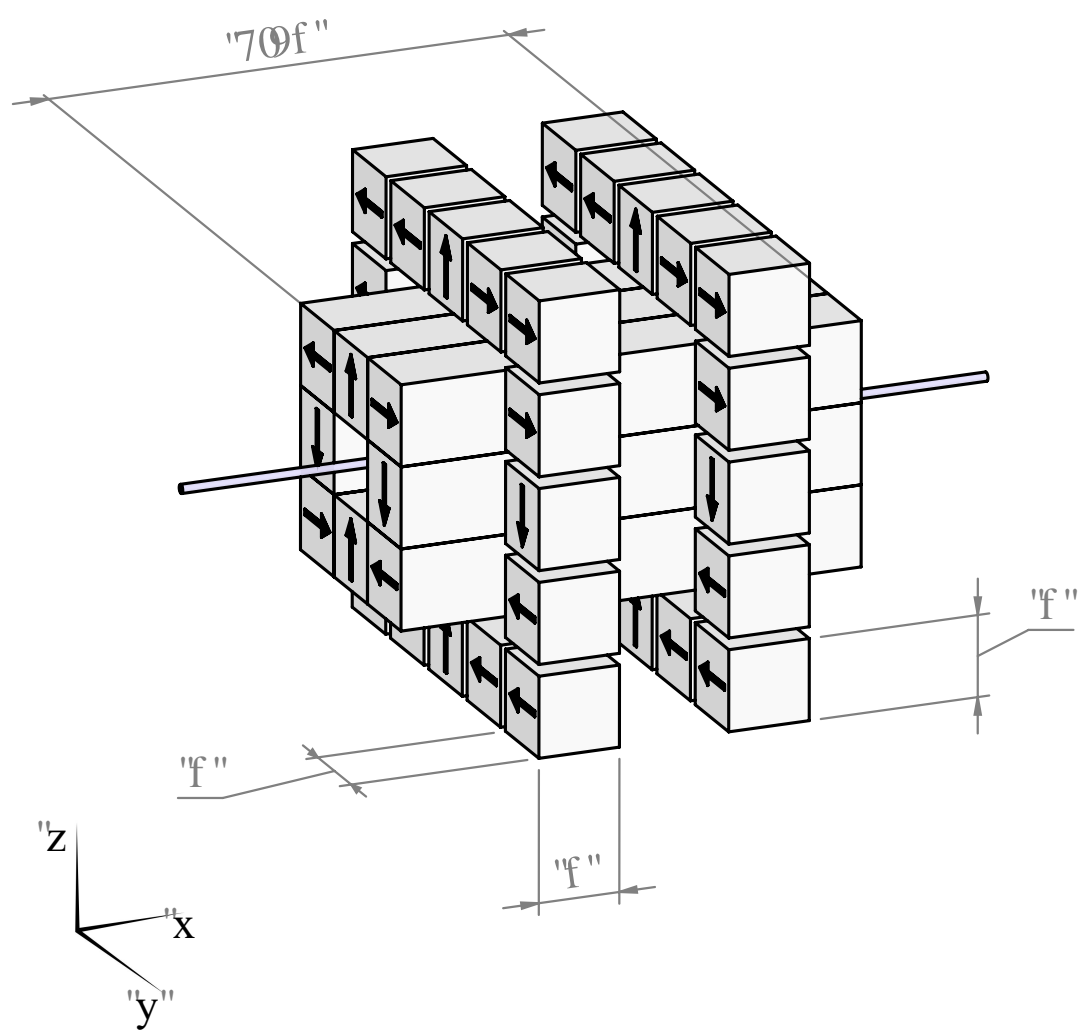

FIGURE 4.1 - Schematic view of the Dumbbell-Halbach configuration which is a combination of the Pseudo-Halbach consisting out of 8 bar magnets and 2 movable shimming rings, each one consisting out of 16 cubic magnets. The capillary tube in the bore contains the sample liquid. In the experimentally tested configuration, $d$ was chosen $7 \mathrm{~mm}$. 


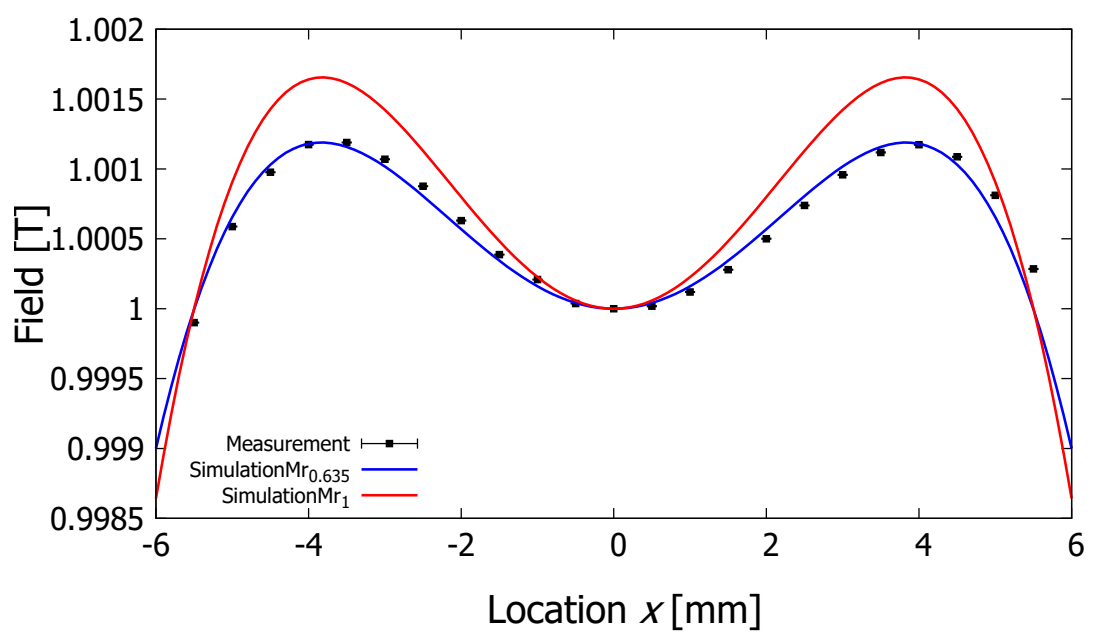

FIGURE 4.2 - Measured and simulated field profile of the Dumbbell-Halbach. The red graph shows the simulated field-profile of the Dumbbell-Halbach if all magnets have the same magnetization. The black points are the measured values of our experimental magnet system. To simulate the effect of the ferrofluid in our system, the measured and simulated field of the Dumbbell-Halbach need to match. Therefore, we used a different magnetization of the shimming-rings in a new simulation, in order to fit the simulation with our measurements. With a ring-magnetization of $0.635 \mathrm{M}_{\mathrm{s}}$ (blue), the simulation shows the same result as our experimental data.

A Hall sensor from Projekt Elektronik GmbH (Teslameter 3002/Transverse Probe $\mathrm{T}_{3}-1,4-5,0-70$ ) in combination with a motorized linear stage (Thorlabs, $\mathrm{DDSM}_{5} \mathrm{O} / \mathrm{M}$ ) has been used to measure the magnet configuration in a temperature stable environment $\left( \pm 0.5^{\circ} \mathrm{C}\right)$. In order to estimate and simulate the amount and magnetization of the ferrofluid to shim the field, we resimulated the measured field profile of our assembled magnet configuration.

With a reduced saturation magnetization of the shimming rings in our simulation to $0.635 \mathrm{M}_{\mathrm{s}}$, the simulation matches the measured profile (Fig4.2).

\subsubsection{Microfluidic shimming chips}

The shimming chips (schematic drawing in Fig.4.3) have been 3D-printed (Frosted Detail Plastic, Shapeways). In the center of the chip (yellow in Fig.4.3) there is a 


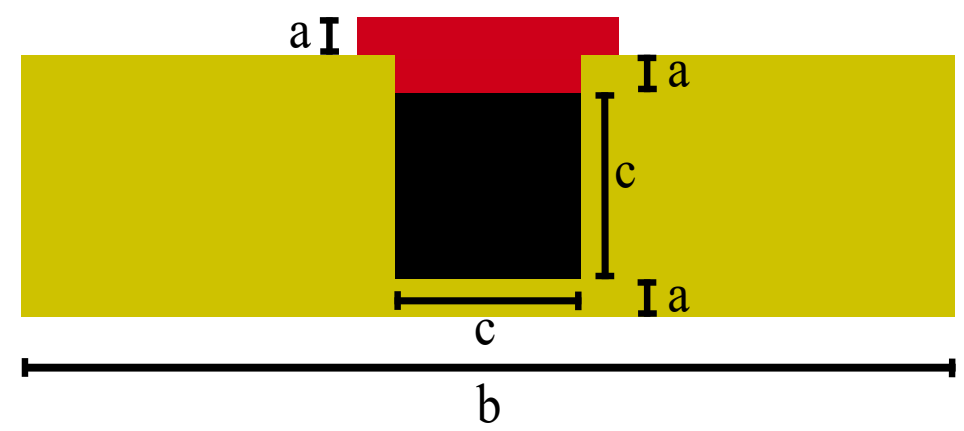

FIGURE 4.3 - Schematic cross-section of the microfluidic shimming chip, consisting out of the main chip (yellow), cap (red) and ferrofluid (black). The edge length of the cubic cavity (c) was varied from $0.6 \mathrm{~mm}$ to $1.0 \mathrm{~mm}$, while the wall thickness and cap has been kept constant $(a=0.2 \mathrm{~mm})$. The chip has a width of 6 mm (b).

cubic cavity which is filled with an oil-based ferrofluid (black in Fig.4.3, purchased from Ferrotec GmbH, EMG series). To keep the ferrofluid in place during the filling process, a small magnet has been placed under the chip. With a lid (red in Fig.4.3), which is glued on top of the chip, it is ensured that the ferrofluid stays within the container when exposed to a magnetic field. To shim the magnetic field of the Dumbbell-Halbach, two chips have been inserted in the bore of the magnet. The containers, filled with ferrofluid, are placed on the top and the bottom side of the inside of the bore, right above and underneath the magnet centre. In the horizontal direction the cavity of the ferrofluid chip was aligned, as shown in Fig. 4.4, with the valley of the field profile (shown in Fig.4.2).

\subsubsection{VSM-measurements ferrofluid}

The measurements have been done with the VSM Model 10 Mark II from Microsense. We confirmed the, from the company mentioned saturation magnetization of each ferrofluid within an error of $4 \%$. With an external field of $1 \mathrm{~T}$, the field strength of the Dumbbell-Halbach, all ferrofluids reach their saturation magnetization (Fig.4.5) . 


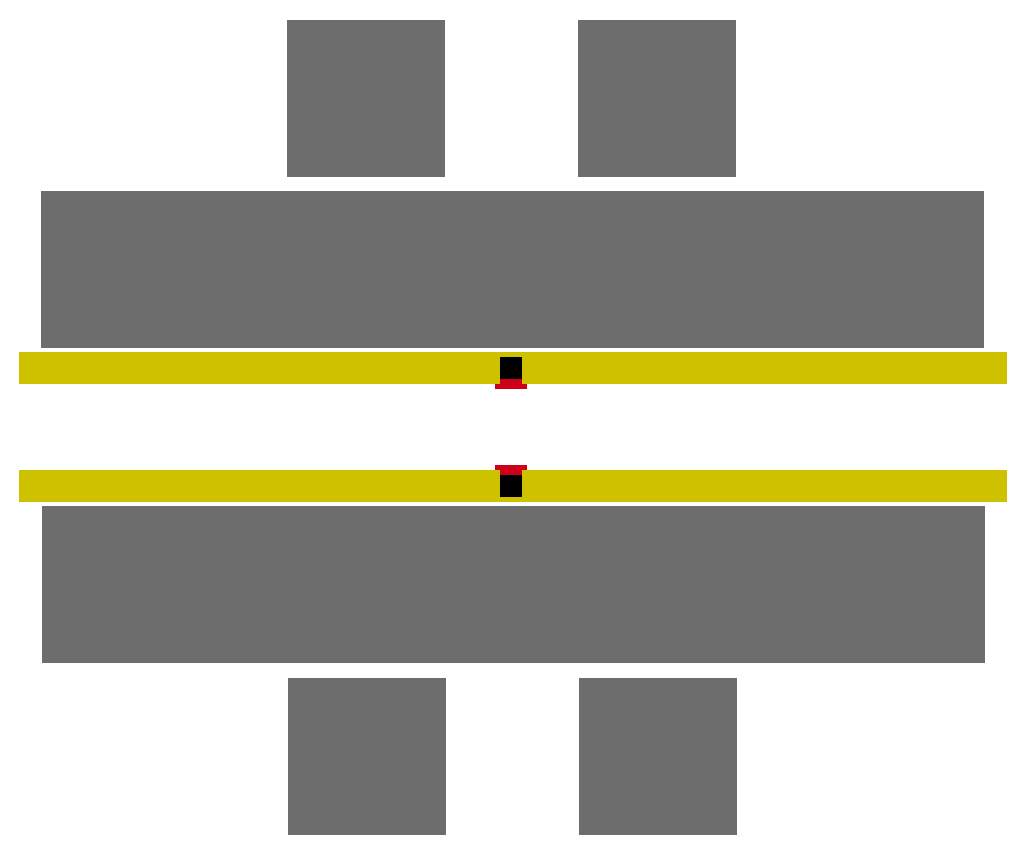

FIGURE 4.4 - Schematic cross-section of the microfluidic shimming chip, inserted in the Dumbbell-magnet. Due to the aluminium frame which is necessary to keep the magnets in place, the chips have a distance of $1.0 \mathrm{~mm}$ to the bar-magnet within the bore. To be able to adjust the chip location along the bore, the total length of the chips is $10 \mathrm{~mm}$ longer as the bar magnets.

\subsection{Results and Discussion}

\subsubsection{Ferrofluidic shimming chips - different saturation magnetization}

We used a cubic container with an edge length of $1 \mathrm{~mm}$ in the microfluidic chip to test the effect of the saturation magnetization of the ferrofluid. In our simulation we used 5 different saturation magnetizations ranging from $11 \mathrm{mT}$ to $99 \mathrm{mT}$. We tuned the distance of the mechanic shimming rings, so that the field maxima is at $-3.8 \mathrm{~mm}$ and $3.8 \mathrm{~mm}$ from the center to ensure a feasible field difference between the field maxima and the field minima in the center. Note that this is not the optimized configuration of the Dumbbell-Halbach. In this way, we ensured that the difference can be minimized with the $44 \mathrm{mT}$ ferrofluid. We normalized the field of the simulation result and the measurement with the field at the location $-4 \mathrm{~mm}$ 


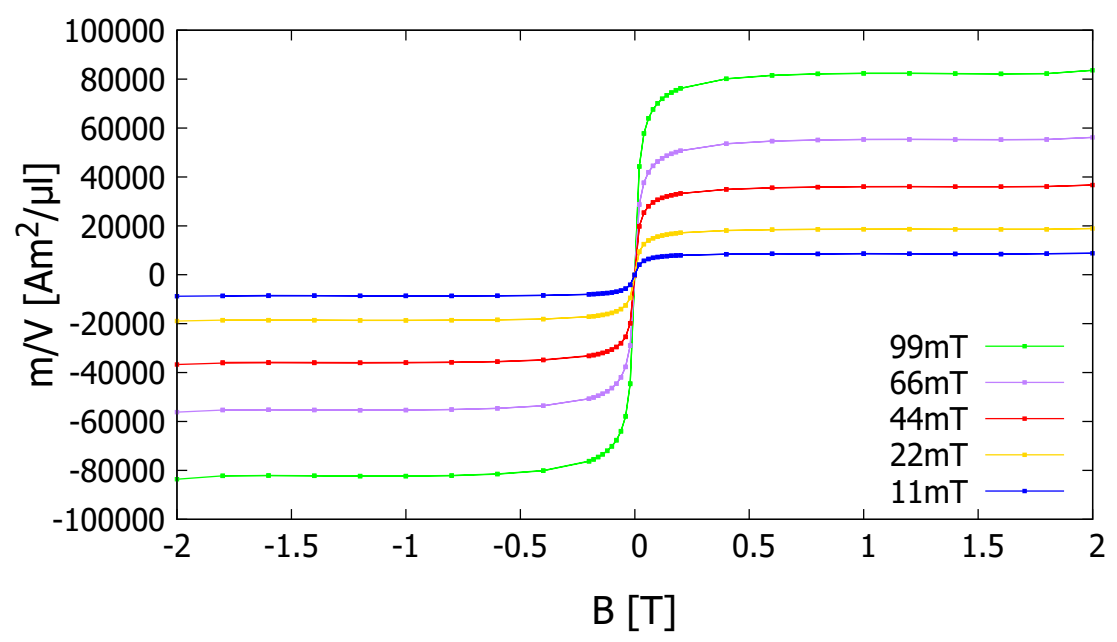

FIGURE 4.5-Hysteresis loop of EMG series ferrofluid obtained from Ferrotec $\mathrm{GmbH}$. With an external field of $1 \mathrm{~T}$, all ferrofluids reach their saturation magnetization of $11 \mathrm{mT}, 22 \mathrm{mT}, 44 \mathrm{mT}, 66 \mathrm{mT}$ and $99 \mathrm{mT}$ within an error of $4 \%$.

TABLE 4.1 - Homogeneity of shimmed Dumbbell-Halbach using ferrofluids with different remanent magnetizations in a $1 \mathrm{~mm}^{3}$. The homogeneity is calculated over a length of $11 \mathrm{~mm}$ horizontally along the center line of the magnet core.

\begin{tabular}{lrrrrrr}
\hline \hline Ferro-fluid [mT] & 0 & 11 & 22 & 44 & 66 & 99 \\
\hline Measured [ppm] & 434 & 329 & 187 & 111 & 179 & 662 \\
Simulated [ppm] & 435 & 346 & 265 & 162 & 241 & 498 \\
\hline \hline
\end{tabular}

to have a clearer presentation of how the field-profile changes with increasing the saturation magnetization of the ferrofluid (Fig.4.6).

With an increasing saturation magnetization of the ferrofluid, the total magnetic field in the center has been increased. The homogeneity could be improved from $162 \mathrm{ppm}$ to $111 \mathrm{ppm}$ using the $44 \mathrm{mT}$ ferrofluid compared to the magnet without shimming-chip. The simulated lines in Fig.4.6 do not always match exactly with the measurements, which is due to a slight difference between the magnetic moment taken in the simulations and the actual material properties (see Table 4.1). The general trends in the measured data are nevertheless represented well by the simulations. 


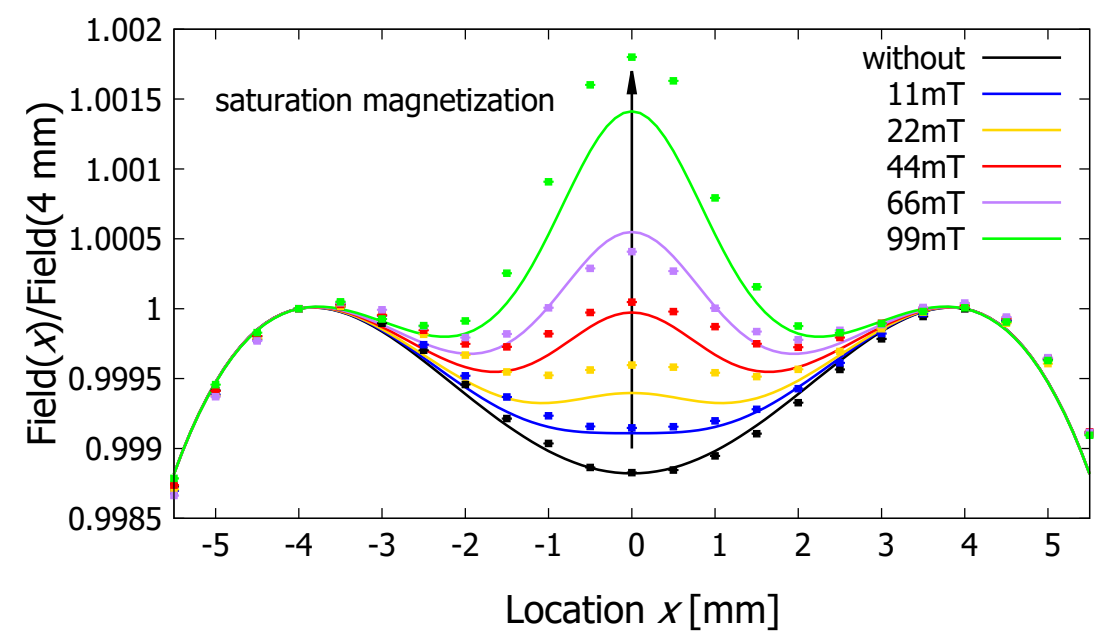

FIGURE 4.6 - Simulated and measured magnetic field profile as a function of the horizontal position along the central line through the magnet core. The different colors correspond to chips with ferrofluids of different magnetization. Dots are measurements, lines are simulations. The ferrofluid can be used to shim the field, increasing the saturation magnetization of the field results in a higher additional field. With the ferrofluid ( $44 \mathrm{mT})$, the profile shows the lowest field difference between field in the center and the field maxima.

\subsubsection{Ferrofluidic shimming chips - different volumes}

We used different cubic container-sizes, ranging from an edge length of $0.6 \mathrm{~mm}$ to $1.0 \mathrm{~mm}$ filled with the ferrofluid with a saturation magnetization of $44 \mathrm{mT}$. With the shimming rings, we tuned the field maxima to be at $-2.5 \mathrm{~mm}$ and $2.5 \mathrm{~mm}$ from the center to ensure a feasible field difference between the field maxima and the field minima in the center, which can be minimized with an edge length of $0.7 \mathrm{~mm}$ in combination with the $44 \mathrm{mT}$ ferrofluid. We normalized the field of the simulation results and the measurements with the field at the location $-3.25 \mathrm{~mm}$ to have a clearer presentation of how the field profile develops (Fig. 4.7). 


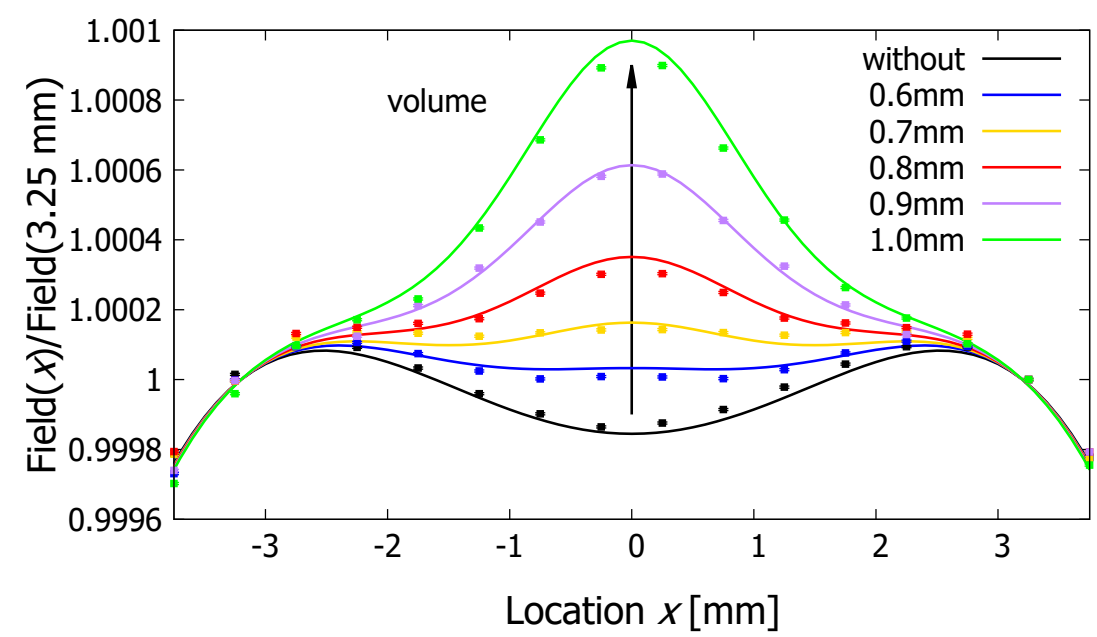

FIGURE 4.7 - Simulated and measured magnetic field profile as a function of the horizontal position along the central line through the magnet core. The different colors correspond to chips of different edge lengths of the cubic chamber, filled with the EMG series $44 \mathrm{mT}$ ferrofluid. Dots are measurements, lines are simulations. The ferrofluid can be used to shim the field, increasing the chamber volume results in a higher additional field, giving an overshoot for the largest volume. The best choice for this ferrofluid is a box with a side of $0.7 \mathrm{~mm}$.

The field in the center is raising with increasing the amount of ferrofluid. The homogeneity gets improved from $86 \mathrm{ppm}$ without shimming chip to $8 \mathrm{ppm}$ using a cubic chamber with an edge length of $0.7 \mathrm{~mm}$ (Table 4.2).

TABLE 4.2 - Homogeneity of shimmed Dumbbell-Halbach using ferrofluids with different volumes (remanent magnetization of $44 \mathrm{mT}$ ). The homogeneity is calculated over a length of $5.5 \mathrm{~mm}$ horizontally along the center line of the magnet core.

\begin{tabular}{lrccccr}
\hline \hline Edge length [mm] & 0 & 0.6 & 0.7 & 0.8 & 0.9 & 1 \\
\hline Measured [ppm] & 86 & 42 & 8 & 61 & 174 & 284 \\
Simulated [ppm] & 86 & 26 & 21 & 81 & 169 & 288 \\
\hline \hline
\end{tabular}




\subsubsection{Discussion}

The measurements for the two different options of implementing microfluidic elements with ferrofluids show that the method is feasible for shimming a small permanent magnet. There are a few aspects that can be improved for further investigations. Our measurements deviate from our simulation results, which can be caused by several factors. A first point of importance is that in the simulation we have used a model of permanent magnets to represent the ferrofluidic elements. Since our magnetic field is mainly present in one direction, we assumed a fully saturated magnetization of our ferrofluid in the same direction as the main magnetic field, without including the field in other directions. For better simulation results, a FEM simulation should be done to mimic the exact behavior of the ferrofluid. Finally, the design of the microfluidic chip can be improved. 3 D-printing is an elegant way to combine fast prototyping with acceptable dimension-tolerances. However, it cannot compete with modern micromachining techniques, where accuracies in the $\mu \mathrm{m}$-range are possible, using state-of-the art lithography methods. It should also be noted that this technology (and admittedly, also $3 \mathrm{D}$ printing methods) allow much more complex microfluidic designs than the simple rectangles and cubes we have applied in this study for proof of principle. Exploiting these techniques to their full potential will enable a very versatile route towards optimized, passively shimmed magnets of very small size.

\subsection{Conclusions and Outlook}

We successfully demonstrated the use of ferrofluid in a microfluidic chip to improve the field homogeneity of a permanent magnet system. We chose 2 ways to change the magnetic moment of the shimming chip: The saturation magnetization and the volume of the ferrofluid. As a proof of principle we improved the homogeneity of our magnet configuration over a length of $11.0 \mathrm{~mm}$ from 434 to $111 \mathrm{ppm}$ with adjusting the saturation magnetization and from $86 \mathrm{ppm}$ to $8 \mathrm{ppm}$ over a length of $5.5 \mathrm{~mm}$ with adjusting the volume. In future studies, following adjustments could be done to improve the shimming result. Instead of one chamber, multiple chambers could be filled with ferrofluid, the saturation magnetization of each one could be tuned to the needed value. The chambers could come in a grid-like arrangement or with shapes and locations, optimized for the specifically needed compensation field. In case there is enough space for multiple microfluidic-chips, they could be stacked on top or next to each other. The shimming by ferrofluids need not be only passive. For active shimming applications a micromixer could be used to adjust the ferrofluid/solvent ratio within the chamber (on chip or separately) to change magnetic moment to the needed level. Another possibiliby would be to adjust the volume of the chambers via microactuator (e.g. electrostatic, pneumatic, microfluidic, piezoelectric...) in order to change the magnetic moment. In order to reach a higher fabrication accuracy, the chip-material or chip processing method could be changed to micromachining, glass capilaries or more accurate $3 \mathrm{D}$-printing techniques. 


\title{
Chapter 5
}

\section{Magnetic Field Strength Improvement for Lorentz Actuation of a $\mu$-Coriolis Mass Flow Sensor}

\begin{abstract}
In this paper we present and compare three different magnet configurations for Lorentz actuation of a $\mu$-Coriolis mass flow sensor. The first configuration consists out of 2 cylindrical magnets, the second is based on a Halbach ring, and the third configuration consists of a single cubic magnet. The magnetic field strength of each configuration is simulated. The Halbach configuration shows a magnetic field strength of $0.3 \mathrm{~T}$, the single cubic configuration reaches $0.25 \mathrm{~T}$. The two cylindrical magnets have the lowest field with $0.05 \mathrm{~T}$. The stray field is significantly lower for the Halbach configurations compared to the other two configurations. All configurations were used for Lorentz actuation of a $\mu$-Coriolis mass flow sensor and the frequency response was measured. The magnitude transfer between the actuation and induction voltages for the cubic and Halbach configurations show a transfer around $26 \mathrm{~dB}$ higher than the cylindrical configuration. The phase transfers for the Halbach and Cubical configurations are according to simulation. For the cylindrical configuration, the EMF signal is too weak to overcome the crosstalk between the actuation and induction voltages.
\end{abstract}

The work in this chapter is a team effort. My contribution includes the modeling of the magnetic field, magnet-assembling and experimental validation of the magnitude and phase transfer.

This chapter is based on (Schut et al., 2020) 


\subsection{Introduction}

Haneveld et al. (Haneveld et al., 2010) first presented a magnetically actuated $\mu$-Coriolis mass flow sensor, as opposed to electro-statically actuated $\mu$-Coriolis devices presented by Enoksson et al. (Enoksson et al., 1997) and Sparks et al. (Sparks et al., 2003). The operation principle is shown in Figure 5.1. The sensor consists of a rectangular channel loop. The channel is fixed at the in-/outlet channel sections, as depicted in the figure. Conventionally, the sensor is brought into resonance by feeding a current $\mathbf{i}$ through a metal track on top of the channel. Lorentz forces are generated in the sections of channel perpendicular to a magnetic field B. These forces induce displacement of the channel. An induction voltage is generated on a second metal track. The two tracks are connected through a closed amplification loop which brings the sensor into resonance in the Twist mode with actuation angle $\theta_{\mathrm{a}}$ (Figure 5.1a). When a fluid flows through the micro-channel with a mass flow rate $\phi_{\mathrm{m}}$, Coriolis forces are induced in the channel section as indicated in Figure 5.1. This causes additional vibration in the Swing mode with detection displacement $\Delta z_{\mathrm{d}}$ (Figure 5.1b). The ratio $\Delta \hat{z}_{\mathrm{d}} / \hat{\theta}_{\mathrm{a}}$ is a measure for the flow. Additionally, one can obtain the density of the fluid flowing though the channel from the actuation resonance frequency.

Groenesteijn et al. (Groenesteijn et al., 2012) have presented improved magnet configurations for actuation of a $\mu$-Coriolis mass flow sensor. Miniature magnets are placed on-chip and with their magnetic poles all in the same direction. This showed improved field strengths but has large disadvantages, since magnets have to be somehow mounted on-chip without damaging the sensor device itself and/or without detaching over time. In this paper, new magnet configurations are presented which give a large increase in the generated magnetic field strength. Three configurations have been chosen and compared. The first uses the field between two parallel cylindrical magnets, the second uses the field in close proximity to a cubic magnet and the third uses the field within a Halbach ring based configuration. The Halbach configuration is a special magnet arrangement which reduces the stray field while enhancing the field at the ring-center. The Halbach magnet configuration has been invented by K. Halbach (Halbach, 1979, 1980) and is still used today, e.g. for several machines (Raich and Blümler, 2004) or mobile NMR applications (Zhu and Howe, 2001).

\subsection{Methods}

\subsubsection{Device \& Fabrication}

The $\mu$-Coriolis mass flow sensor used during the measurements presented in this paper was fabricated by Surface Channel Technology (SCT) (Groenesteijn et al., $2017 a, b)$. A simplified schematic representation of the fabrication process is displayed in Figure 5.2. First, a layer of low-stress silicon-rich silicon nitride (SiRN, Thickness: $500 \mathrm{~nm}$ ) is deposited on a Silicon wafer by Low-Pressure Chemical Vapour Deposition (LPCVD), see Figure 5.2a. On top of this, a layer of 

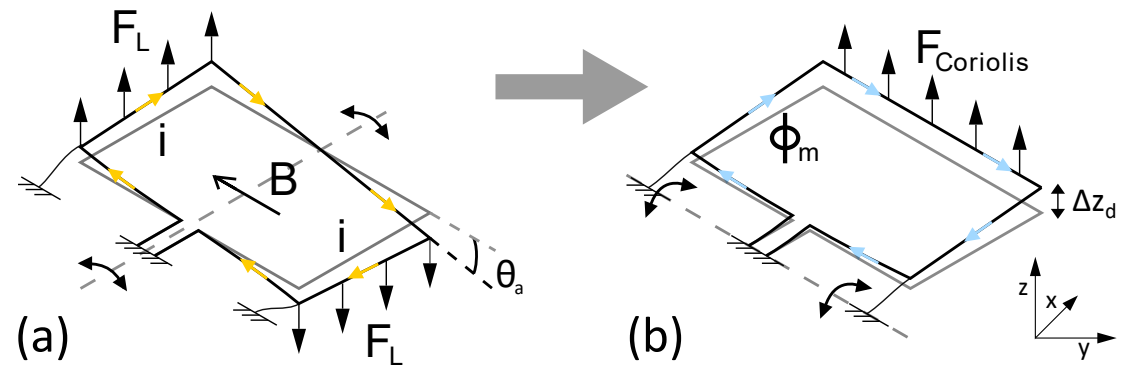

FiguRE 5.1 - Operation principle of a $\mu$-Coriolis mass flow sensor. (a): The channel loop is brought into resonance in the Twist mode through Lorentz force $F_{L}$ resulting from a magnetic field $B$ and a varying current $i .(b)$ : A mass flow $\phi_{m}$ through the channel induces vibration in the Swing mode through Coriolis force $F_{\text {Coriolis. }}$.

silicon di-oxide $\left(\mathrm{SiO}_{2}\right)$ is deposited (LPCVD, Thickness: $500 \mathrm{~nm}$ ), serving as a hard mask. Slits of $5 \mu \mathrm{m} \times 2 \mu \mathrm{m}$ are etched through both layers by plasma etching, see Figure 5.2b. Then the layer of $\mathrm{SiO}_{2}$ is removed and a channel is formed by semi-isotropically etching silicon through the slits, see Figure 5.2c. This is done by plasma etching as well. The channel is closed by conformally depositing another layer of low-stress SiRN, see Figure 5.2d. Metal tracks are patterned on top of the channel (Figure 5.2e), these are used for actuation and readout of the sensor. Following this, openings are etched through the nitride and finally the channel is released by isotropic etching of silicon by an $\mathrm{SF}_{6}$ plasma. A typical cross-section of a surface channel made by this technology is displayed in Figure 5.3.

Figure 5.4 shows an SEM image of the sensor. Multiple metal tracks can be seen on the channel, a.o. for capacitive readout and temperature measurement. In this paper only two metal tracks are actually used: one to provide the current for actuation of the sensor and one for measurement of the induction voltage generated by vibration of the channel.

\subsubsection{Frequency response}

As described earlier, the $\mu$-Coriolis mass flow sensor is actuated by means of Lorentz forces. Figure 5.5 shows a schematic representation of the sensor and closed amplification loop which is used to bring it into resonance. There are two metal tracks on top of the microfluidic channel of the sensor. An actuation voltage $v_{\text {act }}$ is supplied to one track. Due to vibration of the channel, an electromotive force $(\mathrm{EMF})$ is generated on the other track. This EMF $\left(\nu_{\mathrm{EMF}}\right)$ is then fed into an amplifier circuit. The amplified signal is fed back to the actuation track of the sensor. This closed loop circuit drives the sensor into resonance.

The vibrations of the sensor during actuation can be described by a second 
Chapter 5-Magnetic Field Strength Improvement for Lorentz Actuation of a 62
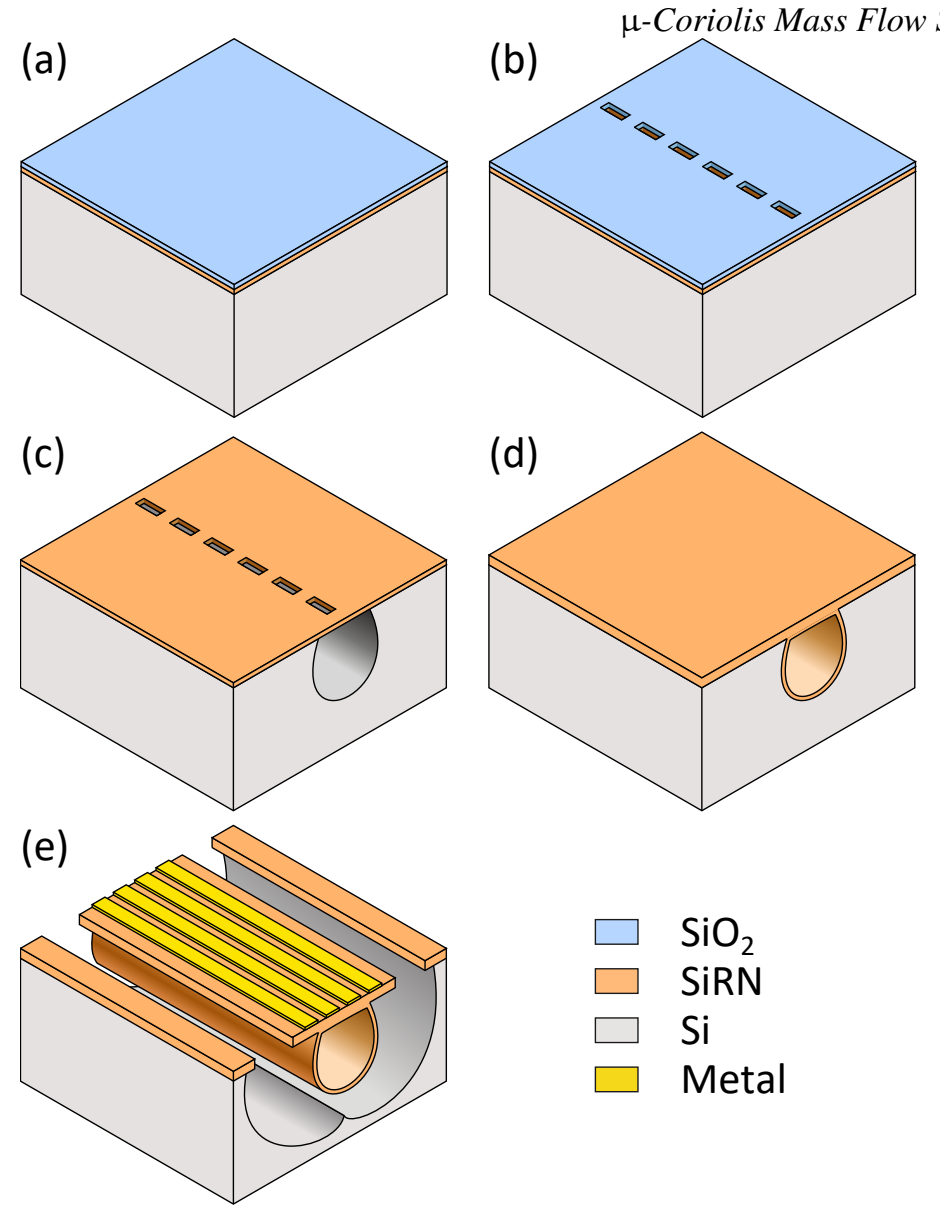

FIgURE 5.2 - Fabrication process for Surface Channel Technology. (a): Silicon wafer with $500 \mathrm{~nm} \mathrm{SiRN} \mathrm{and} 500 \mathrm{~nm} \mathrm{SiO}{ }_{2}$. (b): Patterning of slit openings. (c): Removal of $\mathrm{SiO}_{2}$ hard mask and forming of the surface channel. (d): Closing of the channel. (e): Patterning of metal and release of the channel.

order differential equation:

$$
J \theta^{\prime \prime}(t)+R \theta^{\prime}(t)+S \theta(t)=\tau_{\text {ext }}(t)
$$

Where $J$ is the moment of inertia, $R$ is the damping coefficient, $S$ is the torsional spring constant, $\tau_{\text {ext }}(t)$ is the external torque induced by Lorentz forces and $\theta(t)$ is the actuation mode angle (see Figure 5.1). $\tau_{\text {ext }}(t)$ can be expressed as:

$$
\tau_{\text {ext }}(t)=\frac{B L d}{R_{\text {track }}} \cdot v_{\text {act }}(t)
$$




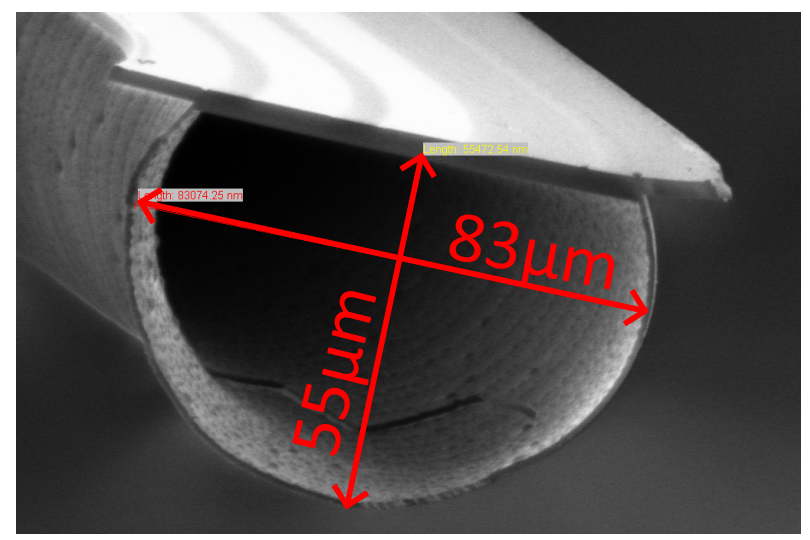

FIGURE 5.3-Typical cross-section of a 'Surface Channel' (Groenesteijn et al., 2017a).

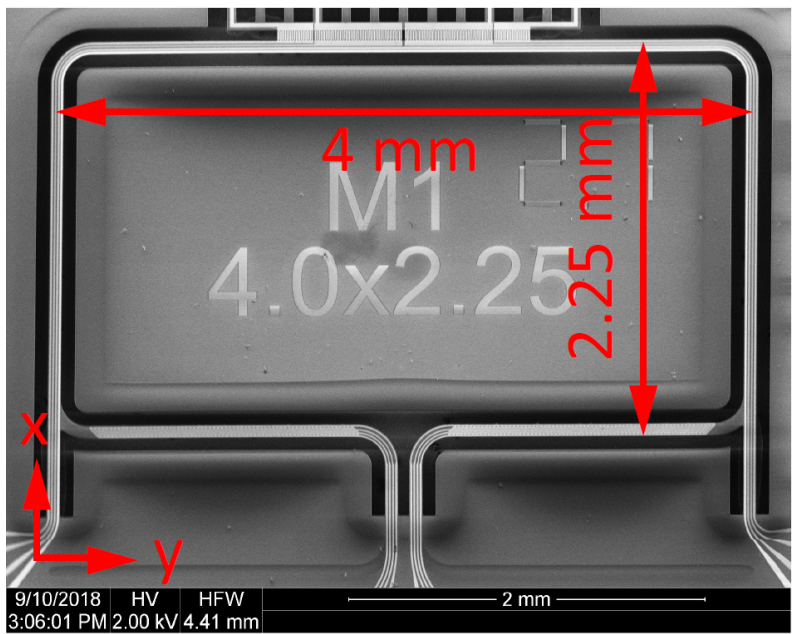

FIGURE 5.4-SEM image of the sensor type used for all simulations and measurements in this paper. 

64

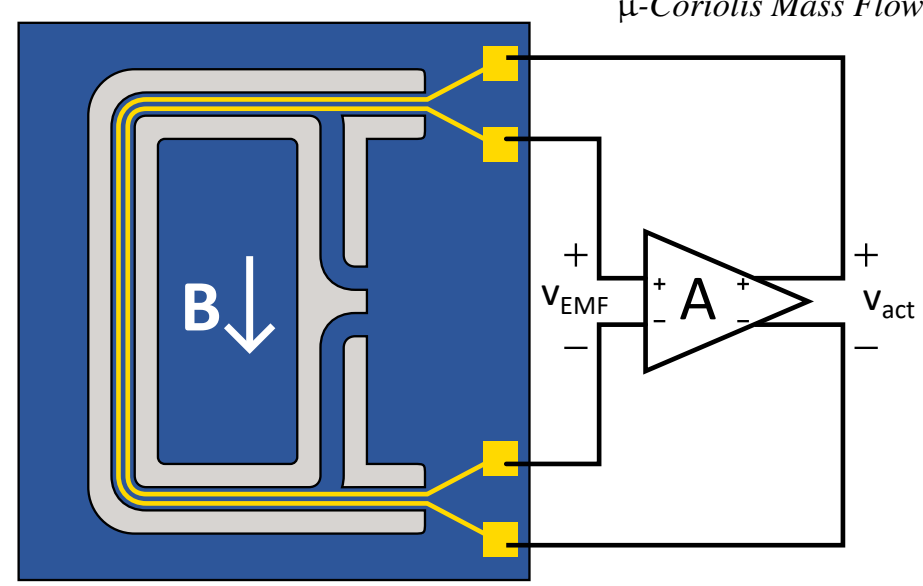

FIGURE 5.5 - Schematic representation of the actuation loop which is used to bring the Coriolis flow sensor into resonance. Where $v_{E M F}$ is the generated electromotive force and $v_{\text {act }}$ is the actuation voltage used to generate Lorentz forces in combination with a magnetic field $\mathbf{B}$.

Where $B$ is the magnetic field strength, $L$ is the length of metal track where Lorentz forces are generated, $d$ is the distance between these track sections and $R_{\text {track }}$ is the resistance of the metal track. The induction voltage generated on the second metal track is dependent on the velocity of the channel in the $z$ direction:

$$
\nu_{\mathrm{EMF}}=2 B L \cdot z^{\prime}(t)
$$

This can then be related to the the actuation angle $\theta$ (for small value of $\theta$ ):

$$
\begin{aligned}
z(t)=\frac{d}{2} \cdot & \sin (\theta(t)) \approx \frac{d}{2} \theta(t) \\
V_{\mathrm{EMF}}(\omega) & =2 B L \cdot j \omega Z(\omega) \\
& =B L d \cdot j \omega \theta(\omega) \\
\theta(\omega) & =\frac{V_{\mathrm{EMF}}(\omega)}{j \omega B L d}
\end{aligned}
$$

Now converting Equation 5.1 to the frequency domain and substituting Equations 5.2 and 5.6 :

$$
\begin{aligned}
J(j \omega)^{2} \theta(\omega)+R j \omega \theta(\omega)+S \theta(\omega) & =\tau_{\text {ext }}(\omega) \\
\theta(\omega) \cdot\left((j \omega)^{2} J+j \omega R+S\right) & =\frac{B L d}{R_{\text {track }}} \cdot V_{\text {act }}(\omega) \\
\frac{V_{\mathrm{EMF}}(\omega)}{j \omega B L d} \cdot\left((j \omega)^{2} J+j \omega R+S\right) & =\frac{B L d}{R_{\text {track }}} \cdot V_{\text {act }}(\omega)
\end{aligned}
$$


Finally, the transfer function from $V_{\text {act }}$ to $V_{\mathrm{EMF}}$ becomes:

$$
\begin{aligned}
\frac{V_{\mathrm{EMF}}}{V_{\mathrm{act}}} & =\frac{j \omega B^{2} L^{2} d^{2}}{R_{\mathrm{track}} \cdot\left((j \omega)^{2} J+j \omega R+S\right)} \\
& =\frac{j \omega B^{2} L^{2} d^{2} / S}{R_{\mathrm{track}} \cdot\left(1+j \frac{\omega}{\omega_{0}} \frac{1}{Q}+\left(j \frac{\omega}{\omega_{0}}\right)^{2}\right)}
\end{aligned}
$$

Where $\omega_{0}$ is the resonance frequency and $Q$ is the quality factor:

$$
\begin{aligned}
& Q=\frac{\sqrt{S J}}{R} \\
& \omega_{0}=\sqrt{\frac{S}{J}}
\end{aligned}
$$

Equation 5.8 is used in combination with the simulated magnetic field strengths to generate simulated transfer curves for the various magnet configurations. These are compared with transfer curves measured using a gain-phase analyzer. The measurement setup is effectively as shown in Figure 5.5 where the amplifier circuit is then substituted by an HP 4194A gain-phase analyzer.

\subsubsection{Magnetic simulation and assembly}

Three different magnet configuration have been simulated and measured.

The simulations have been done using CADES simulation software, entirely described by Delinchant et al. (Delinchant et al., 2007). Magnetic interactions are modeled with the MacMMems tool, which uses the Coulombian equivalent charge method to generate a semi-analytic model. This model is used by the CADES framework (component calculator, component optimizer) to calculate the designs.

Three different magnet configurations have been simulated and measured as shown in Figure 5.6.

The cylindrical configuration consists of two cylindrical, axially magnetized

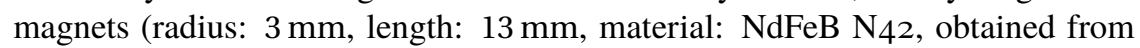
www.supermagnete.nl) arranged parallel with a distance of $19 \mathrm{~mm}$ between them. The chip has been placed in the middle with an offset of $3 \mathrm{~mm}$ in the $z$-direction, as shown in Figure 5.6a. Our Halbach-ring (Figure 5.6b) consists of 8 magnets (material NdFeB N45, obtained from www.supermagnete.nl). 4 of them, placed in the corners, cubic with an edge length of $7 \mathrm{~mm}$, the other ones are $7 \mathrm{~mm} \times 7 \mathrm{~mm} \times 14 \mathrm{~mm}$. The cubic configuration (Figure 5.6c) consists of a cubic magnet with an edge length of $12 \mathrm{~mm}$ (material $\mathrm{NdFeB} \mathrm{N} 45$, obtained from www.supermagnete.nl) placed parallel, directly above the chip. In all configurations the channels of interest have a length of $2.25 \mathrm{~mm}$ and are located at $\mathrm{y}=$ $-2 \mathrm{~mm}$ and $2 \mathrm{~mm}$. The magnets can be placed in a holder surrounding the chip allowing for simple plug and play operation. 
Chapter 5-Magnetic Field Strength Improvement for Lorentz Actuation of a 66

Top view $\mu$-Coriolis Mass Flow Sensor

(a)

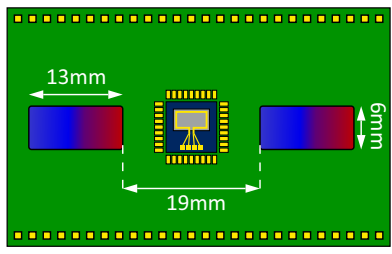
Isometric view

(b)
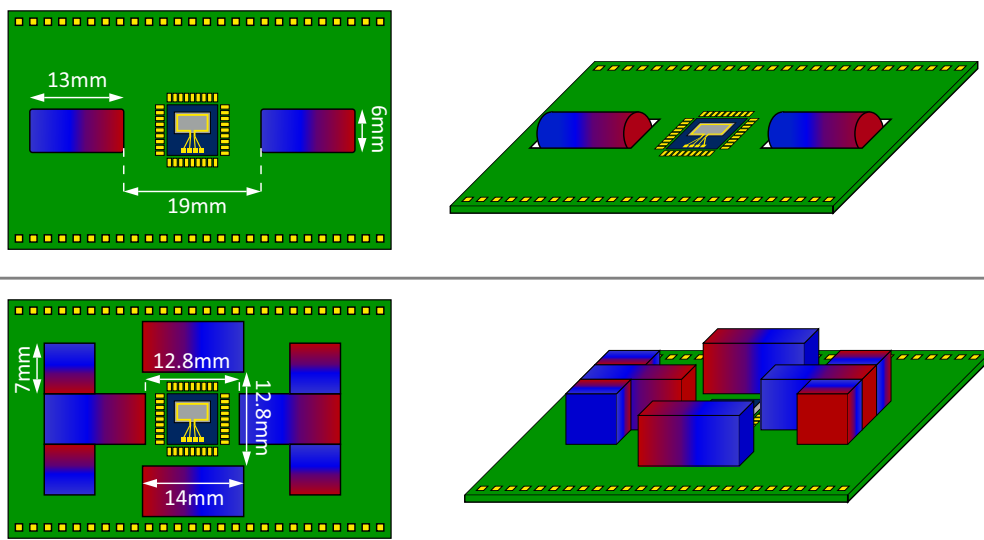

\section{.}
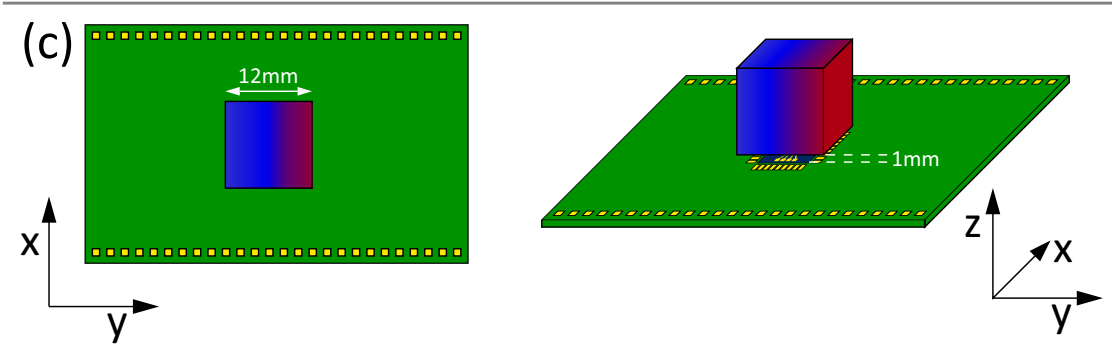

FIGURE 5.6- $-\mu$-Coriolis sensor chip on PCB with different magnet configurations. a) Conventional $13 \mathrm{~mm} \times 6 \mathrm{~mm}$ cylinder magnets, b) Halbach configuration with cube magnets with sides of $7 \mathrm{~mm}$. c) Single large $12 \mathrm{~mm}$ cube magnet. Red and blue indicate the north and south pole of the magnets respectively.

\subsection{Results}

The magnetic field strength of three different magnet configurations have been simulated. For all configurations the $y$-field shows a uniform profile along the channel, see Figure 5.7. The Halbach configuration reaches a strength $0.3 \mathrm{~T}$, the single cuboid configuration $0.25 \mathrm{~T}$. The cylindrical configuration has the lowest field with $0.05 \mathrm{~T}$.

The $x$-field of the Halbach and Cube configuration shows a slope of $-0.01 \mathrm{~T} / \mathrm{mm}$, the cylinder $-0.003 \mathrm{~T} / \mathrm{mm}$, see Figure 5.8. The slope of each configuration scales with the reached absolute field. The field is anti-symmetric around $x=0$ due to symmetry of the magnet configurations. This means additional Lorentz forces are generated in the channel section perpendicular to the $y$-axis. Due to the anti-symmetry of the $x$-field this will cause additional vibration in the actuation mode. The Coriolis mode is unaffected by this field.

The stray magnetic field strengths of the three configurations were simulated 


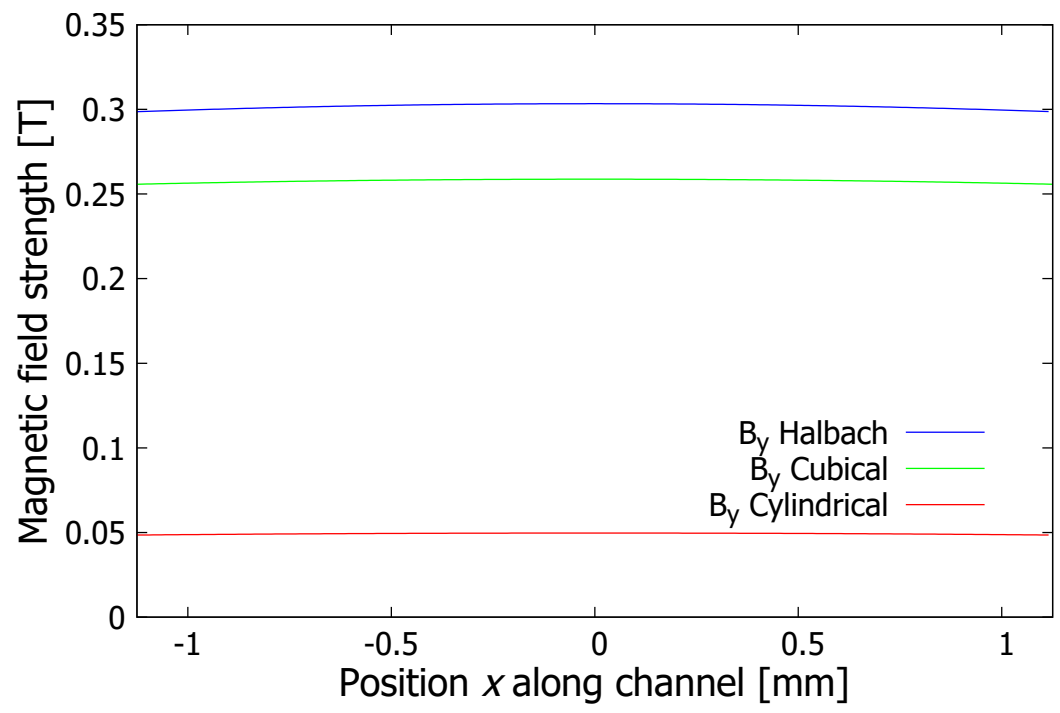

FIGURE 5.7 - Simulated magnetic field strength in the y-direction along the side 2. $25 \mathrm{~mm}$ channel section (figure 5.6). Halbach configuration (blue) shows the highest field strength $\left(0.3^{T}\right)$ followed by the single cubic magnet (green), arranged on top of the chip $(0.25 T)$. Using two cylindrical magnets (red) results in a field strength of $0.05 T$.

\begin{tabular}{l|l} 
Configuration & Stray field $[\mathrm{T}]$ \\
\hline Cylindrical & 0.025 \\
Halbach & 0.006 \\
Cube & 0.081
\end{tabular}

TABLE 5.1 - Stray magnetic field strength along y-axis of the three configurations at a distance of $10 \mathrm{~mm}$ from the outer magnet surface.

at a distance of 10mm from the outer magnet surface, see Table 5.1. The Halbach configuration shows the lowest stray field strength of $0.006 \mathrm{~T}$, compared to $0.025 \mathrm{~T}$ for the cylindrical configuration and $0.081 \mathrm{~T}$ for the cubic configuration.

The magnitude and phase transfer between the actuation voltage $v_{\text {act }}$ and induction voltage $v_{\mathrm{EMF}}$ was measured (as described in section 5.2.2) for the three magnet configurations in Figure 5.6. The magnitude and phase transfer are displayed in Figures 5.3a and b respectively. The analytical curves are generated using equation 5.8 in combination with the simulated magnetic field strengths for the three configurations (see Figure 5.7). For both figures the $x$-axis is centered around the resonance frequency, which lies around $3.8 \mathrm{kHz}$ varying slightly with temperature, pressure and from chip to chip. 


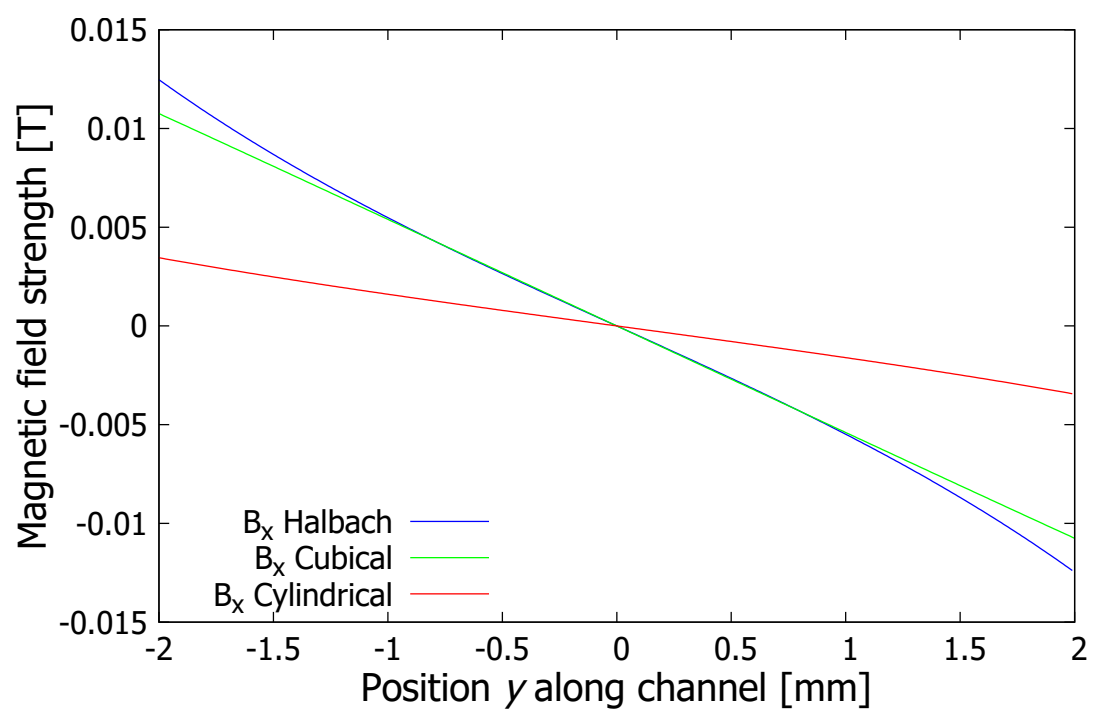

FIGURE 5.8 - Simulated magnetic field strength in the $x$-direction along the top 4 mm channel section (figure 5.4). All configurations show a linear gradient of the $x$-field along the channel. The Halbach and Cube configuration have slope of -o.o1T/mm, the cylinder-o.oozT/mm.

The measured magnitude transfers for the 3 configurations are; Halbach: $-59.1 \mathrm{~dB}$, Cubic: $-58.0 \mathrm{~dB}$, Cylindrical: $-84.6 \mathrm{~dB}$. As expected, both the Halbach and single cube configuration show a higher magnitude transfer than the cylindrical configuration, around $26.5 \mathrm{~dB}$. For the cubic configuration, this corresponds well with the analytical curve. The measured value is slightly higher than simulated. This could be due to deviation of the position of the magnet along the $z$-axis where it is actually closer to the sensor chip than simulated. Other causes could be a deviation in the quality factor or resonance frequency of the Coriolis flow sensor. For the Halbach configuration, the simulated value of the magnitude transfer lies $4.4 \mathrm{~dB}$ higher than the measured value. This is most likely due to misalignment of the magnets in the Halbach configuration. Since the fields of the magnets in the configuration oppose each other, it is difficult to accurately place and align them. Misalignment of the sensor chip to the centre of the magnets along the $z$-axis (where the magnetic field is the strongest) could be another cause of the lower measured transfer.

The phase transfer of all the configurations should in principle be a curve going from $+90^{\circ}$ to $-90^{\circ}$, eventually going back to $+90^{\circ}$ due to cross-talk via parasitic capacitance between the actuation and induction tracks. At resonance, the phase shift should be $0^{\circ}$. However, since the signal in case of the cylindrical configuration is so small, the phase shift is heavily affected by cross-talk (see Figure 5.3b). The 

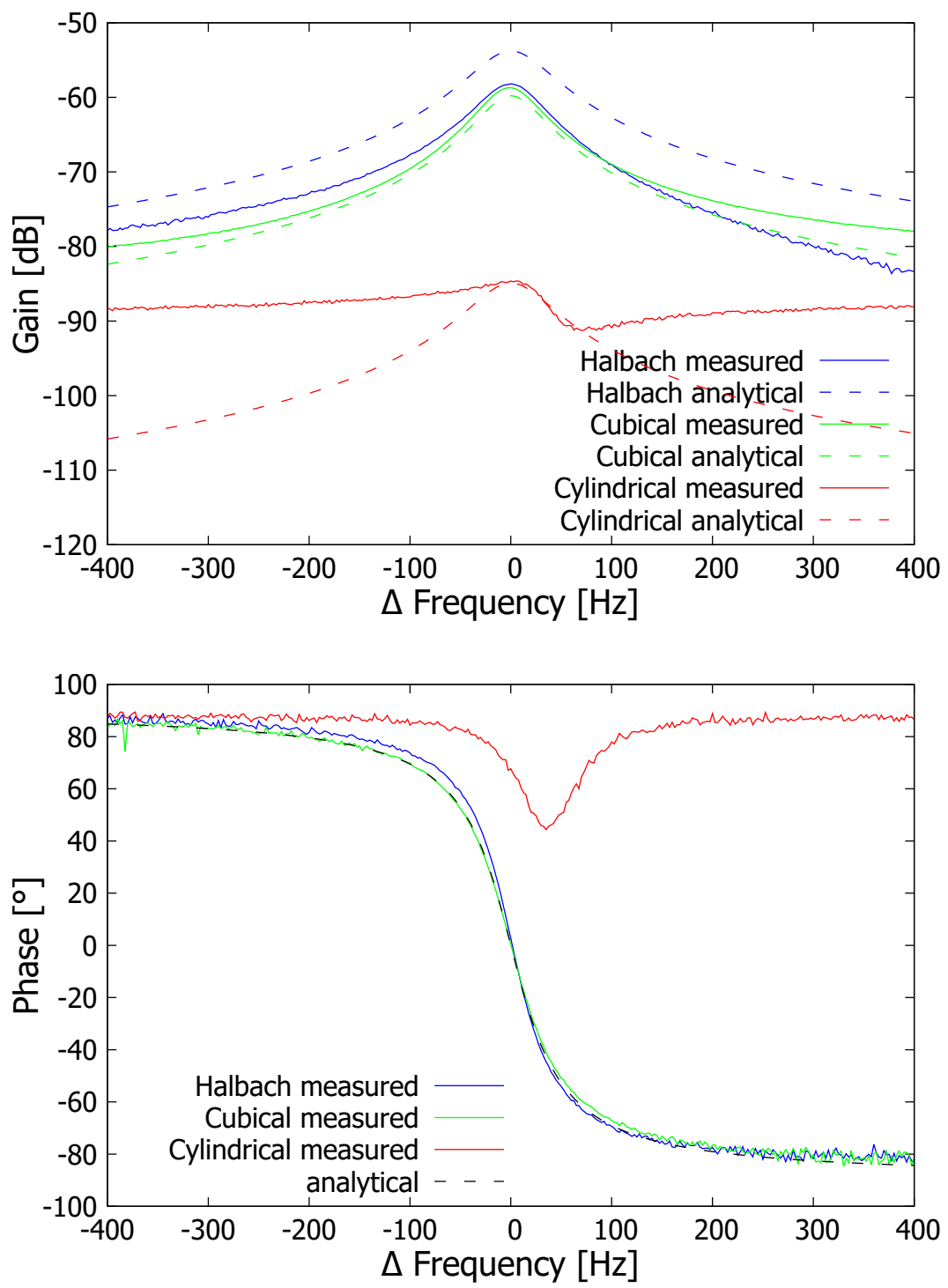

FIGURE 5.9-Magnitude and phase transfers between the actuation voltage $v_{\text {act }}$ and induction voltage $v_{E M F}$. Top: The measured magnitude transfers for the 3 configurations are; Halbach: $-59.1 \mathrm{~dB}$, Cubic: $-58.0 \mathrm{~dB}$, Cylinders: $-84.6 \mathrm{~dB}$. Bottom: The measured phase transfer of the Halbach and cubic configurations goes from $+90^{\circ}$ to $-90^{\circ}$, as simulated. For the cylinder configuration the phase only reaches around $45^{\circ}$ before going back up to $90^{\circ}$. 
Chapter 5-Magnetic Field Strength Improvement for Lorentz Actuation of a 70

$\mu$-Coriolis Mass Flow Sensor

phase shift does not even reach $0^{\circ}$. The EMF signal is too small to overcome the cross-talk via parasitic capacitance between the two metal tracks. We can see a similar effect in the magnitude transfer of the cylindrical configuration where the resonance peak barely reaches out above the noise floor. To stably bring the sensor into resonance, it is critical that this $0^{\circ}$ point can be detected. For the other two configurations, the phase transfer is as expected. For these, the sensor should be able to be brought stably into resonance with an amplification loop with relatively low gain. This can enhance the signal-to-noise ratio of the overall sensor. Especially for fluid density measurements this can improve the accuracy significantly, since the resonance frequency is directly related to the density of the fluid flowing though the channel.

\subsection{Conclusions}

The Halbach configuration shows the highest simulated field strength $(0.3 \mathrm{~T})$ followed by the single cubic magnet positioned above the chip $(0.25 \mathrm{~T})$. Two cylindrical magnets generate a field strength of $0.05 \mathrm{~T}$, see Figure 5.7. All configurations show a linear gradient of the $x$-field along the channel. The Halbach and Cube configurations have a slope of $-0.01 \mathrm{~T} / \mathrm{mm}$ and the cylindrical configuration shows a slope of $-0.003 \mathrm{~T} / \mathrm{mm}$. Both the cubic and cylindrical configurations show a constant $z$-field offset $(-0.115 \mathrm{~T} \&-0.01 \mathrm{~T}$ respectively) along the channel, the Halbach configuration shows no offset (Figure 5.8). The Halbach configuration shows the lowest stray field, see Table 5.1.

Magnitude transfer measurements between the actuation and induction voltages for the cubic and Halbach configurations show a transfer $26 \mathrm{~dB}$ higher than the cylindrical configuration, see Figure 5.3. Phase transfer measurements show that the Halbach and cubic configurations generate transfer plots according to simulation. The low EMF signal amplitude for the cylindrical configuration causes the phase to only reach down to $45^{\circ}$ before going back up to $90^{\circ}$. This means the Halbach and cubic configurations are more suitable for use with an amplification loop to bring it into stable resonance, which is important for overall performance of the $\mu$-Coriolis flow sensor. To conclude, the Halbach configuration has the most promising results regarding its field strength and stray-field but is hard to assemble due to the repelling forces of the magnets. Therefore we would suggest to use a single large cubic magnet which combines a high magnetic field and a practical way to assemble the final sensor. 


\title{
Chapter 6
}

\section{Trajectory Deflection of Spinning Magnetic Microparticles, the Magnus Effect at the Microscale}

\begin{abstract}
The deflection due to the Magnus force of magnetic particles with a diameter of $80 \mu \mathrm{m}$ dropping through fluids and rotating in a magnetic field was measured. With the Reynolds number for this experiment around 1, we found trajectory deflections of the order of $1^{\circ}$, in agreement with the measurement error in theory. This method holds promise for the sorting and analysis of the distribution in magnetic moment and particle diameter of suspensions of microparticles, such as applied in catalysis, or objects loaded with magnetic particles.
\end{abstract}

The work in this chapter is a team effort. My contribution includes the modeling of the magnetic field and magnetic force.

\subsection{Introduction}

A rotating object moving through a medium experiences a Magnus force that is perpendicular to both the axis of rotation and the direction of motion (Magnus, 1853). This effect is well known in ball sports. The Magnus force is an inertial effect, and therefore is most effective for large objects moving at high velocity, such as soccer balls (Kensrud, 2010) or planets forming in a protoplanetary disc (Forbes, 2015). In these situations the flow is normally turbulent, characterized by much higher linear $\left(\mathrm{R}_{\mathrm{e}}\right)$ and rotational Reynolds number $\left(\mathrm{R}_{\mathrm{o}}\right)$. In this paper, we investigate the Magnus force for laminar flow conditions, at Reynolds numbers close to unity. The experiments were performed with spheres of only $80 \mu \mathrm{m}$ in diameter, rotating less

This chapter is based on (Solsona et al., 2020) 


\section{Chapter 6 - Trajectory Deflection of Spinning Magnetic Microparticles, the}

72 Magnus Effect at the Microscale

than five revolutions per second and moving at about one centimeter per second through water.

The Magnus force has technological relevance, since it can be used for magnetic separation of microparticles (Rem et al., 2002). In contrast to magnetic separation by force gradients, Magnus separation is performed in a uniform field. The Magnus force separates primarily on particle size, since the deflection of the particles is proportional to the square of their diameter. When increasing the rotation frequency of the field, particles with low magnetic moment can no longer follow the field. So by tuning the rotation frequency, one can independently separate on magnetic moment as well. The theory of the Magnus effect at low Re has been studied in detail (Rubinow and Keller, 1961). It was shown that the Magnus force is linearly proportional to the rotational $\mathrm{R}_{\mathrm{e}}$ up to values in the order of one hundred. However, measurements of the Magnus force are not reported for values below of few hundreds (Rem et al., 2002). A serious experimental complication is that the deflection of the trajectory of the objects is approaching the object size, which complicates analysis. The solution we chose is to reduce the particle size, which also allows us to benefit from microfluidic systems.

Microfluidic technologies have been used extensively to sort cells and microparticles using forces that are a function of combinations of particle properties such as size(Lu and Xuan, 2015; Yamada and Seki, 2006), shape, density(Huh et al., 2007), permittivity, susceptibility and magnetic moment(Amini et al., 2014; Gossett et al., 2010; IV et al., 2015; Sajeesh and Sen, 2013; Whitesides, 2006). The use of these forces in well-controlled laminar fluids with external actuators enable important applications such as sorting of cancer cells ( $\mathrm{Li}$ et al., 2015) or catalyst particles (Solsona et al., 2018). In addition to forces, torques can be applied on particles by rotating fields (Moerland et al., 2019). At low $\mathrm{R}_{\mathrm{e}}$, linear and rotational drag forces balance by the applied torque immediately, resulting in constant linear and angular velocity(Gou et al., 2018; Saffman, 1965; Zhang et al., 2016) and therefore a constant Magnus force.

In the experiment we present here, the force resulting in linear velocity was provided by gravitation and the torque leading to rotational velocity was provided by a rotating magnetic field acting on anisotropic magnetic Janus particles with a diameter of $80 \mu \mathrm{m}$. These particles are in the same size range as fluidic cracking catalyst particles (Solsona et al., 2018, 2019), so that the results are of immediate technological relevance. The resulting deflections of the particles was observed in a microscope at different rotation speeds and medium viscosities, and compared to an approximate model for low $\mathrm{R}_{\mathrm{e}}$.

We show that if the experiment is performed carefully, the deflection due to the Magnus force is measurable for $\mathrm{R}_{\mathrm{e}}$ around unity. As long as the particles rotate, their trajectories are deflected with an angle that is in agreement with theory. These results encourage the application of Magnus separation on the microscale using microfluidic technology. 


\subsection{Theory}

A sphere dropping through a fluid experiences drag forces on its surface. If the velocity is sufficiently low, the relative velocity of the fluid molecules at the surface is zero, and all drag forces are due to shear between the molecules in the fluid itself. At low velocity, the drag force on a small surface element is proportional to the relative velocity of the fluid at small distance from that surface. The velocity of the sphere reaches a maximum when the total drag force integrated over the surface balances the gravitational force.

If the sphere does not rotate, the fluid velocity is mirror symmetric to the vertical axis (the falling direction). All horizontal components of the drag forces compensate each other, all vertical forces add up to a net drag force opposite to the velocity (Fig. 6.1a). If the sphere rotates, the velocity field is modified. At very low rotation velocityvelocities, the field is mirror symmetric with respect to the horizontal plane through the centre of the sphere. All horizontal components of the drag forces above the plane are compensated by opposite horizontal force below that plane. As a result, there is no net horizontal force, (Fig. 6.1b). At higher rotation velocities however, the fluid approaching the bottom of the sphere (the front side) needs a non-negligible distance to accelerate. At the top of the sphere (the back side), the fluid needs a certain distance to decelerate. As a result, the symmetry with respect to the horizontal plane is lost. Horizontal drag force components above the horizontal plane are no longer compensated by components below that plane. Consequently, there is a net drag force component perpendicular to the vertical and the sphere trajectory is deflected from the vertical axis, Fig. 6.1c. This resulting forces was initially discovered be Isaac Newton and two centuries later again by H. G. Magnus (Magnus, 1853). In essence it is an inertial effect, (Barkla and Auchterlonie, 1971; Seifert, 2012) and therefore increases with increasing linear Reynolds number $\left(\mathrm{R}_{\mathrm{e}}=2 u r / v_{f}\right)$ and rotational Reynolds number $\left(\mathrm{R}_{\mathrm{o}}=\Omega * r^{2} / v_{f}\right)$ where $u\left(m s^{-1}\right)$ is the relative linear velocity between the particle and the fluid, $\Omega\left(\operatorname{rad} s^{-1}\right)$ is the angular velocity [in the experimental part, we express the angular velocity in the more intuitive units of revolutions per second (rps), $r(\mathrm{~m})$ is the radius of the particle, and $v_{f}$ is the kinematic fluid viscosity of the fluid $\left(\mathrm{m}^{2} \mathrm{~s}^{-1}\right)$ (Bagchi and Balachandar, 2002; Kray et al., 2012). Many studies have been performed in order to model this force (Beck, 2018). For low Reynolds numbers, Rubinow and Keller (Rubinow and Keller, 1961) model the force as

$$
F_{\text {magnus }}=\pi \rho_{f} r^{3} \Omega \times u,
$$

where $\rho f\left[\mathrm{~kg} \mathrm{~s}^{-3}\right]$ is the fluid density, $\mathrm{r}[\mathrm{m}]$ the radius of the particle, $\omega$ [rad $\left.\mathrm{s}^{-1}\right]$ the angular velocity and $\mathrm{u}\left[\mathrm{ms}^{-1}\right]$ the relative velocity between the particle and the fluid (in the experimental part, we express the rotational velocity in the more intuitive units of revolutions per second [rps]). The force is maximum when the rotation axis is perpendicular to the relative velocity. 
Chapter 6 - Trajectory Deflection of Spinning Magnetic Microparticles, the 74

(a)

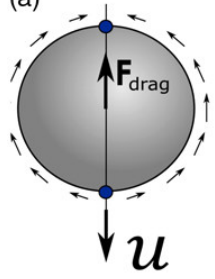

(b)

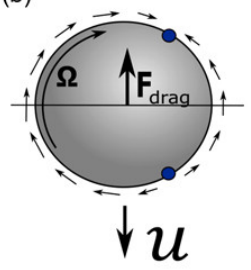

Magnus Effect at the Microscale

(c)

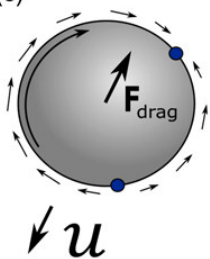

(d)

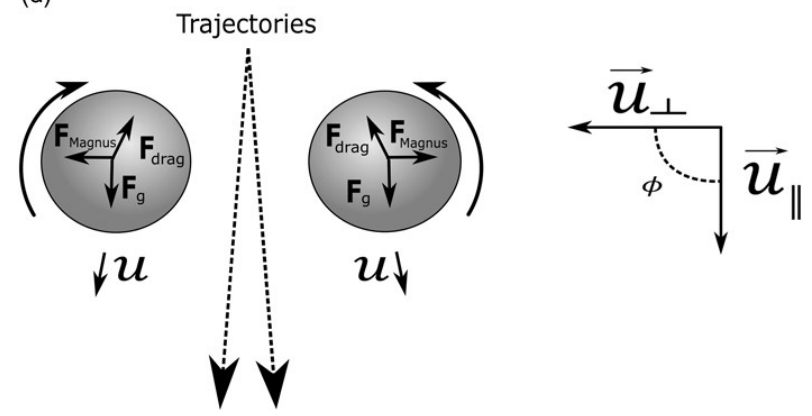

FIGURE 6.1 - Schematic drawing of the principle causing the Magnus force. A sphere dropping through a fluid experiences a drag force due to the shear force between the fluid and the sphere surface. The blue dots indicate the point where the relative fluid velocity along the surface of the sphere is zero. (a) When the sphere does not rotate, the vertical axis is an axis of mirror symmetry (indicated by the line). (b) When the sphere rotates slowly, the flow pattern shifts but remains symmetric with the horizontal plane (indicated as well). There is no net force perpendicular to the falling direction. (c) At higher rotation velocity, the fluid at the front size of the sphere needs a certain distance to accelerate, which shifts the position of zero velocity down. The resulting asymmetry in the pattern leads to a tilt in the drag force. (d) Definition of forces and velocities.

Previous work has experimentally demonstrated the existence of the Magnus force at low $R_{e}$. Oesterle and Dinh (Oesterlé and Dinh, 1998)used metal spheres few centimetres big attached to a thread in order to spin them to quantify the Magnus force at $\mathrm{R}_{\mathrm{e}}$ between 10 and 140. Others (Forbes, 2015; Rem et al., 2002; Tsuji et al., 1985) studied the phenomena at higher $R_{e}$, from 300 to 105 . To the best of our knowledge, the study of the Magnus force has never been performed at Reynolds numbers close to unity. Neither are we aware of studies with microparticles. Experiments with microparticles are challenging, since the Magnus force has a third order dependency on the particle size, equation 1, so the deflection decreases considerably with reduction in particle diameter (Denisov et al., 2018). In order to rotate the particles, a rotating magnetic field was used so that we can apply a torque over a big spatial region (Moerland et al., 2019). The Magnus force is proportional 
to the rotation of the particles (equation 6.1). The rotation velocity is equal to the rotation of the magnet only if the particles can follow the magnetic field. If the magnet rotation speed is too high, the particles only wobble. To estimate the maximum rotation speed, we assume that the particle has a remanent magnetic moment $\mathrm{m}_{\mathrm{p}}\left[\mathrm{Am}^{2}\right]$ and that the field $\mathrm{B}[\mathrm{T}]$ is small compared to the saturation field. Under these conditions we can estimate the maximum torque from

$$
\Gamma \leq m_{p} \times B
$$

A sphere with radius $\mathrm{r}$ rotating in a fluid with viscosity $v \mathrm{f}[\mathrm{Pa} \mathrm{s}]$ at a rotational velocity $\omega\left[\mathrm{rad} \mathrm{s}^{-1}\right]$ experiences a drag torque in the direction opposite to the rotation(Berg, 1993),

$$
\Gamma_{d}=-8 \pi r^{3} \mu_{f} \Omega
$$

By balancing the magnetic and rotational drag torque, we obtain the maximum rotational velocity

$$
\Omega \leq \frac{m_{p}}{r^{3}} \frac{B}{8 \pi \mu_{f}}
$$

In addition to being pulled sideways by the Magnus force, the particles are pulled downwards by gravity as,

$$
F_{g}=\left(\rho_{p}-\rho_{f}\right) g V_{p},
$$

where $\rho_{\mathrm{p}}\left[\mathrm{kg} \mathrm{m}^{-3}\right]$ is the particle mass density, $g\left[\mathrm{~m} \mathrm{~s}^{-2}\right]$ the gravitational acceleration constant and $\mathrm{V}_{\mathrm{p}}\left[\mathrm{m}^{3}\right]$ the volume of the particle. A sphere moving in a fluid encounters a translational drag force in the opposite direction to the movement,

$$
F_{d}=-6 \pi r \mu_{f} u
$$

Balancing the forces, and considering that the magnetic torque is applied perpendicular to $g$, we obtain the velocity of the particle, which we decompose in the translational and perpendicular components, see Fig. 6.1:

$$
\begin{gathered}
u_{\|}=\frac{\left(\rho_{p}-\rho_{f}\right) g V_{p}}{6 \pi \mu_{f} r}, \\
u_{\perp}=\frac{\rho_{f} r^{3} \Omega u_{\|}}{6 \mu_{f} r}
\end{gathered}
$$




\section{Chapter 6 - Trajectory Deflection of Spinning Magnetic Microparticles, the} 76 Magnus Effect at the Microscale

The tilt angle of the trajectory is therefore:

$$
\begin{gathered}
\phi=\tan ^{-1}\left(\frac{u_{\perp}}{u_{\|}}\right) \\
\phi=\tan ^{-1}\left(\frac{\rho_{f} r^{2}}{6 \mu_{f} \Omega}\right) \\
\phi=\tan ^{-1}\left(\frac{r^{2}}{6 v_{f} \Omega}\right) \\
\phi \approx \frac{r^{2}}{6 v_{f} \Omega}
\end{gathered}
$$

The approximation is valid for small angles. When the rotational speed is zero, the particles follow the gravitational force and the tilt angle is zero. The tilt angle increases with increasing sphere radius $r$, and decreases with increasing kinematic fluid viscosity $v\left[\mathrm{f}^{2} / \mathrm{s}\right]$. Small particles with high magnetic moment can rotate faster than big particles with low moment (equation 6.4). The tilt angle is however only dependents on the particle size (equation 6.9). So by selecting combinations of rotating speed $\omega$ and field strength $B$, and filtering out certain tilt angles, we have a method to discriminate particles based on radius, to some extend irrespective of magnetic moment. Next to the Magnus force, the particles will experience a magnetic force along the magnetic field gradient,

$$
F_{m}=m_{p} \nabla B
$$

which will also lead to a tilt angle. Fortunately, since the magnetic moment will align with the field direction, the direction of the gradient is independent on the sign of the field. The sign of the tilt angle however is determined by the rotation direction. So by measuring for both rotations, any tilt due to a magnetic force gradient can be subtracted.

\subsection{Methods}

There are three specifications that the fluidic system must accomplish. First, due to the small deviations expected by the Magnus force and in order to facilitate the following trajectory measurement, the particles should start at very similar positions. Second, the system should be long enough to track long trajectories and third, the particles should be clearly seen through the fluidic system walls. Figure 6.2 shows the $3 \mathrm{D}$ printed fluidic system with 3 inlets and 1 outlet. 2 of the 3 inlets are used to introduce the liquid and the $3 r d$ inlet is used to introduce 


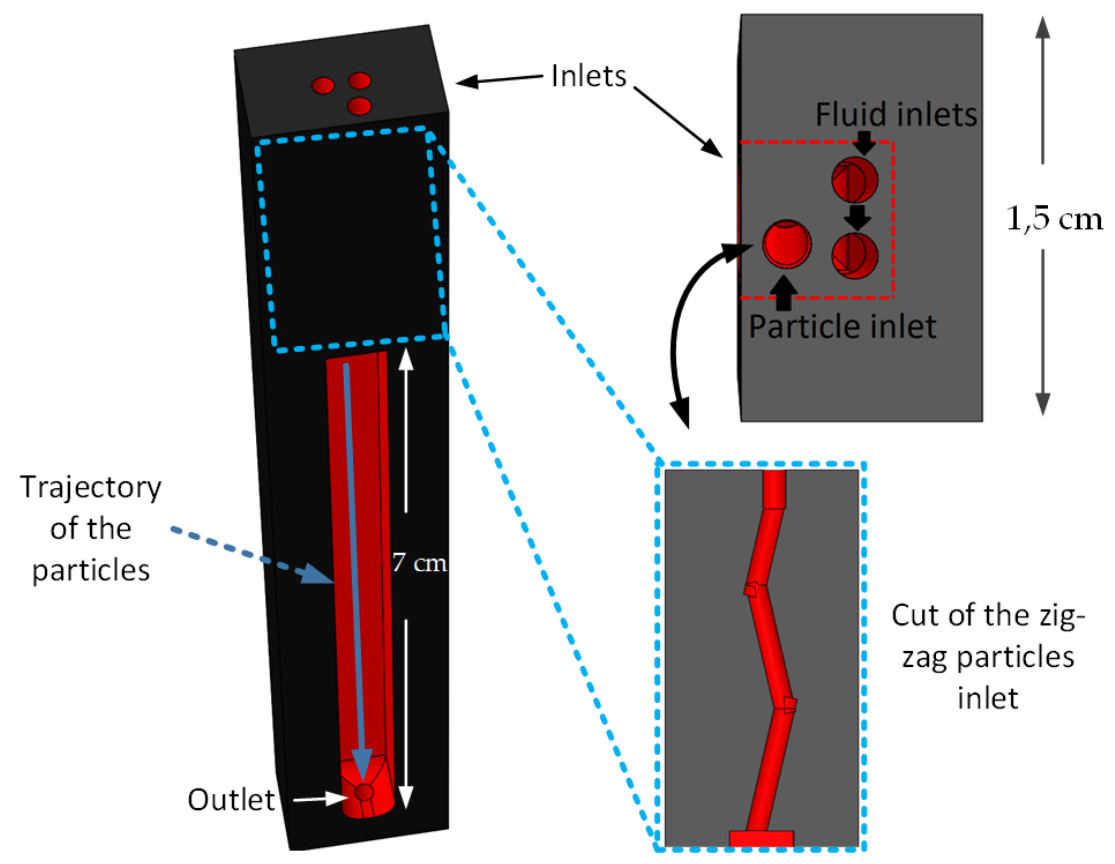

FIGURE 6.2 - (left) Schematic drawing of the fluidic chip showing the 3 inlets and chamber where the particles rotate, (right top) the 3 inlets and (right bottom) cut of the zig-zag particles inlet.

the magnetic particles, also see Figure 6.7 in the appendix. The chamber is 7 $\mathrm{cm}$ long and $0.5 \mathrm{~cm}$ wide and deep. In order to observe the particles and seal the chamber, a glass slide was glued on the front part of the chip, see Figure 6.7 in appendix. To maximize the deflection caused by the Magnus force, the time of the particles inside the chamber should be increased. Therefore, the system will be used with no-flowconditions, letting the particles sink from the inlet to the outlet of the chamber. A very similar particle starting point inside the fluidic system is crucial due to the small deflection expected caused by the Magnus force. Normally, liquid flow, or magnetic, electric or acoustic fields are used to focus the particles inside a microfluidic channel. However, due to the no-flow conditions and the trouble of introducing actuators inside the $3^{\mathrm{D}}$ printed chip, a new method to focus the particles at the same position was developed. Figure 6.2 shows a cut of the particle's inlet of the microfluidic chip where a zig-zag inlet can be seen. As can be seen in Figure 6.8 the appendix, particles were rolling downwards. Although some of the particles started at very similar positions, this varied due to its large sensitivity on other factors such as: the amount of particles arriving to the big chamber at the same time and disturbing each other or by any small flow perturbation caused by the pipetting of the particles inside the inlet. 


\section{Chapter 6 - Trajectory Deflection of Spinning Magnetic Microparticles, the}

78

Magnus Effect at the Microscale

In order to rotate the particles and avoid any attraction to the magnets, the magnetic field that is used should be as strong and homogeneous as possible. Therefore, bigger magnets providing less gradient over the observation area are better than smaller magnets. Also, by placing another magnet on the opposite side of the fluidic chip the gradient, and therefore its magnetic force towards the magnets, will be reduced, see Figure 6.3. Eight N42 magnets $1 \mathrm{~cm}$ wide, $4 \mathrm{~cm}$ deep, $1 \mathrm{~cm}$ long and a magnetization of $1.3 \mathrm{~T}$ along the longest side were purchased from Supermagnete. Each rotating arm consisted of 4 magnets. The field strength in the center between the magnets was $122 \pm 5 \mathrm{mT}$. In order to observe the particle's trajectory a silicon wafer acting as a mirror was glued at a $45^{\circ}$ angle to the fluidic system's wall, see Figure 6.7 in the appendix. Both magnets should rotate at the same speed in order to avoid magnetic field distortion, but due to the fluidic connections and tubing both magnets could not be attached together. Therefore, a new mechanical system was developed to rotate both magnets at the same speed. Figure 6.9 in the appendix shows the system consisting of an electrical motor (Crouzet DC motor model 820580002 ) and a gear box with a reduction ration of 3.4, see Figure 6.7 in appendix. Also, for security reasons, a new system was developed to mount the magnets in the rotating arms. As can be seen in appendix, first the magnets were stuck together with a separator in the middle and subsequently attached together to one of the rotator's arms. Thereafter, the other rotating arm was approached and connected to the second magnet's support, which allows safe separation of both magnets. The magnets repelled each other due to their configuration therefore the second arm was pushed away by the magnets. The separation of the magnets can be adjusted with extra screws in the rotating arms. The rotating speed of the magnets was adjusted controlled by a Conrad PS 405 Pro power supply. The rotation speed was calibrated with a stroboscope (LED-stroboscope HELIO-STROB micro2). A Grasshopper3 $\mathrm{GS}_{3}-\mathrm{U}_{3}-23 \mathrm{~S} 6 \mathrm{M}$ high-speed camera was used to observe and record the trajectories of the particles. The 3 D printed chip was designed in SolidWorks and printed with a Formlabs Form 2 printer.

The particles used in this study are magnetic Janus particles $70-90 \mu \mathrm{m}$ in diameter and purchased from Cospheric. Their core is made of borosilicate and they are half coated with a superparamagnetic material. Some Janus particles were placed between two $5 \mathrm{~mm} \times 5 \mathrm{~mm}$ tape layers and introduced in a Quantum Design PPMS-VSM (Physical property measurement system vibrating sample magnetometer) to measure their magnetic moment. The scan rate was $1.5 \mathrm{mT} / \mathrm{s}$ from $5 \mathrm{~T}$ to $-5 \mathrm{~T}$ and up to $5 \mathrm{~T}$ again at room temperature. Figure 6.10 in the appendix presents the experimentally obtained magnetization of the janus particles. From the magnetization curve, it can be concluded that the janus particles have hysteresis and that their magnetic moment saturates at $\approx 0.5 \mathrm{~T}$ having a susceptibility of $0.06 \mathrm{nAm}^{2} / \mathrm{T}$ and a remanent moment $m_{p}$ is $1.5 e^{-11} \mathrm{Am}^{2}$ per particle. The resulting magnetic force exerted by the magnet on the particles (equation 10) was estimated from the magnet geometry using analytical integration (MacMMems, CADES). We are mostly concerned with variations in the magnetic force for different particle trajectories. A constant force will merely cause an offset that is 


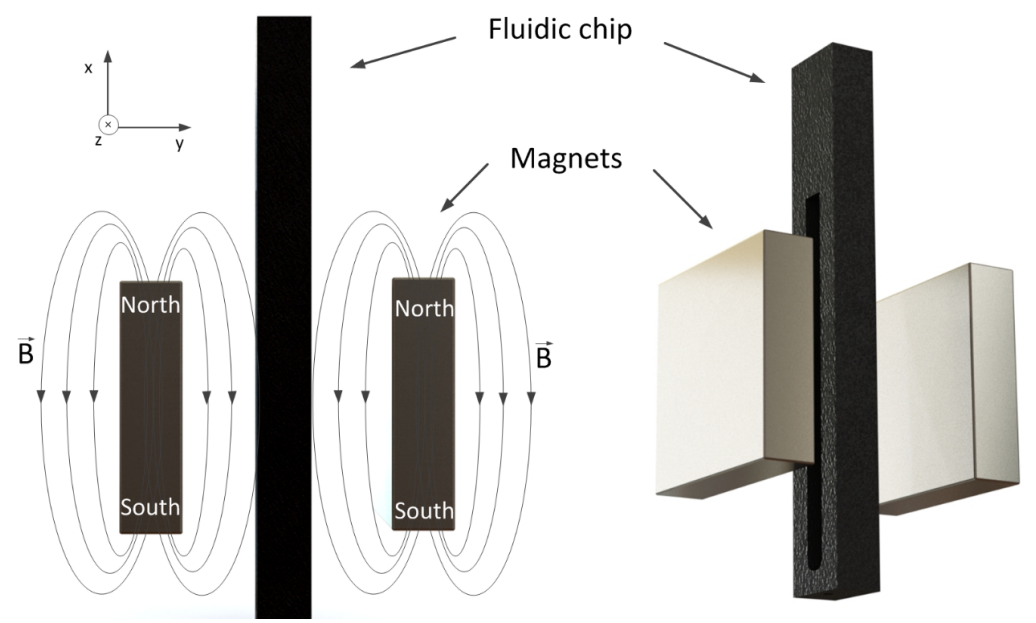

FIGURE 6.3 - (left) Schematic side-view drawing and magnetic field distribution used to rotate the janus particles inside the fluidic chip. (right) Diagonal-view of the magnets configuration.

cancelled by measuring the difference between clock and anti-clockwise rotation. The gradient of the magnetic force in the $z$-direction, so in the same direction as the magnus force, was estimated to be $176 z_{0} \mathrm{nN} / \mathrm{m}$, where $z_{0}$ is the off-center position. When the particles fall exactly along the centre between the magnets, the force and force gradient is zero. Even if the particles are $5 \mathrm{~mm}$ off-axis, the force gradient is only in the order of $1 \mathrm{nN} / \mathrm{m}$. Typical deflection differences due to the Magnus effect are less than $0.1 \mathrm{~mm}$, resulting in maximum magnetic force differences of $0.1 \mathrm{pN}$. The magnetic attraction by the magnets is therefore negligible to the Magnus force itself, which is in the order of $6 \mathrm{pN}$ (DI water with $\mu \mathrm{f}=1 \mathrm{mPa}$ s and $5 \mathrm{rps}$ ). Two solutions were used, DI water and a mix of glycerol and water in a 1:3 volume ratio. Three pipette tips were glued in the three inlets to facilitate the introduction of liquids and particles. The chip was filled with a pipette until the liquid reached the top of the pipette tips. Matlab was used to track the particles trajectories and CADES was used to simulate the magnetic field and magnetic force. Three different experiments were performed where just the liquid viscosity and the rotation speed of the particles were modified. The experimental procedure consisted of different steps. First, the rotation of the magnets was set to a given rotational speed. Second, the janus particles were introduced in the fluidic chip via a pipette. Thereafter, they rolled and fell into the main channel. Third, their trajectories over $1 \mathrm{~cm}$ length inside the fluidic system were recorded and subsequently analysed. Six different steps in each experiment were performed, three clockwise and three anti-clockwise. The center of the trajectories was manually centred in o in order to visualize the difference between experiments. 


\subsection{Results and Discussion}

Figure 6.4 shows the experimentally obtained angles of the trajectories of the particles and their cumulative distribution function (CDF) assuming normal distributions. In the top Figure 6.4 a clear difference can be observed between particle trajectories when the magnet is rotating either clock or anti-clockwise, in case we used water $\left(v_{f}=1.010^{-6} \mathrm{~m}^{2} / \mathrm{s}\right)$ and $5 \mathrm{rps}$ as rotational speed. The deflection of the trajectories agrees with theory: particles rotating clockwise moved to the left and particles rotating anti-clockwise moved to the right. The experimental difference between the mean deflection angles for the two rotation directions was $1.2 \pm 0.3^{\circ}$. This is within error margin in agreement with the calculated angle difference of $1.2^{\circ}$ (equation 6.9). The middle histogramm in Fig. 6.4 shows the same experiment but using $40 \%$ of the rotational speed ( $2 \mathrm{rps}$ ). The measured angle difference $\left(0.42 \pm 0.15^{\circ}\right)$ again agrees with the calculated angle of $0.49^{\circ}$. When we used a liquid with more than twice the viscosity $\left(v=2.310^{-6} \mathrm{~m}^{2} / \mathrm{s}\right)$ and low rotational speed, $2 \mathrm{rps}$, the difference between particles rotating in both directions drops below the measurement error. This was an expected result since the calculated angle dropped until $0.21^{\circ}$. When we increased the rotational velocity to $5 \mathrm{rps}$, most of the particles no longer were able to follow the magnetic field rotation. Assuming the magnetic field to be in the order of $122 \mathrm{mT}$, the maximum rotation velocity in the high viscosity medium ( $\mu=2.3 \mathrm{mPas}$ ) is, according to equation 6.2 , in the order of $78 \mathrm{rps}$, so we should have observed the particles completely following the magnetic field and a deflection similar to $0.52^{\circ}$. We speculate that most of the particles did not have enough magnetic material and therefore not enough torque to follow the magnetic field. In order to obtain larger deflections and more feasible sorting systems, larger rotation speeds are needed which can be obtained by increasing the magnetic field strength inside the fluidic system by placing the magnets closer together. It should be noted that the Magnus force is smaller than typical magnetic forces on microparticles in microfluidic devices. Comparing different systems using magnetic fields to sort particles, it can be observed that similar forces are accomplished $(1-35 \mathrm{pN})$, however, with smaller particles $(1-7.3 \mu \mathrm{m})(\mathrm{Chung}$ et al., 2013, 2016; Kong et al., 2010; Liu et al., 2009; Mirowski et al., 2005; Zhu et al., 2012). The experimental system was constructed for minimal magnetic field gradients, and therefore required big magnets. The size can be optimized, however, especially if we allow magnetic field gradients on top of the Magnus force for additional sorting. 

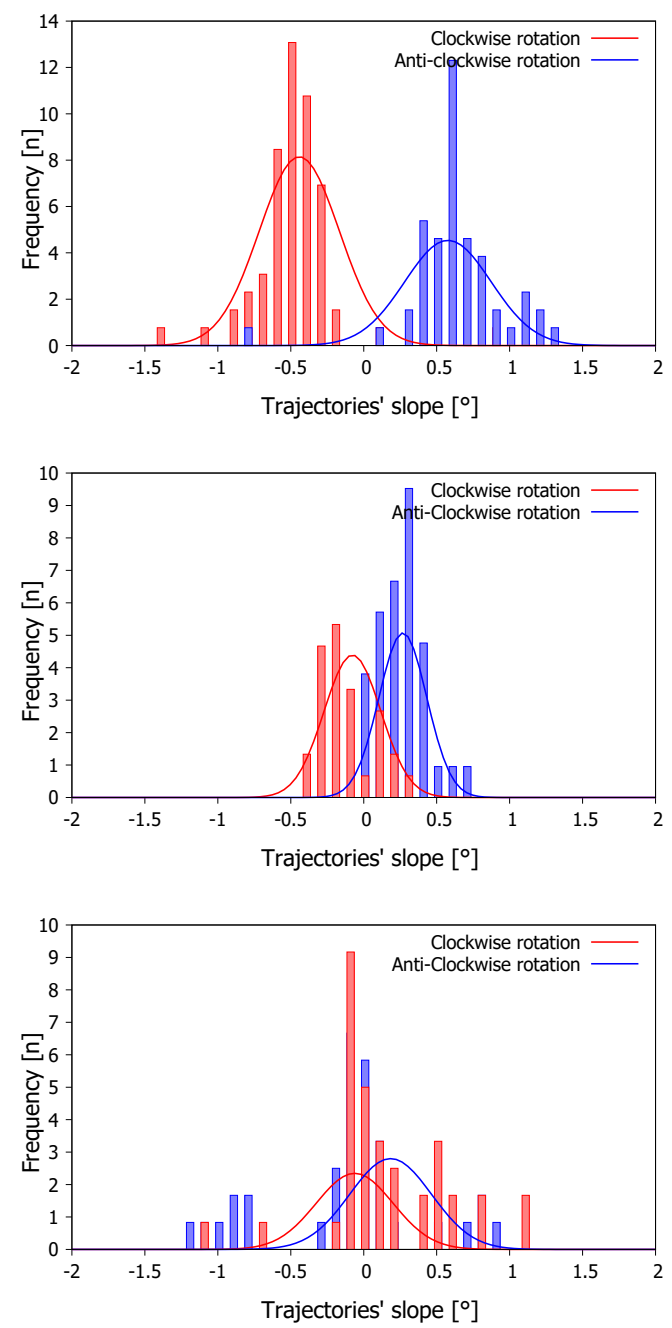

FIGURE 6.4 - Histograms and their cumulative distribution function (CDF) of the trajectory slope angles in the z-direction of particles rotating clockwise (red) and anti-clockwise (blue) (top) with low viscosity (water) and $5 \mathrm{rps}$, (mid) low viscosity (water) and 2 rps, and (bottom) high viscosity (mix of water and glycerol $3 / 1, v / v)$ and 2 rps 


\subsection{Conclusions}

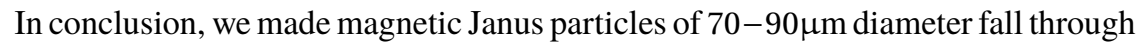
a liquid with varying viscosity in the presence of a rotating magnetic field with varying angular velocity. The Reynolds number for the particle movement was as low as 1 . Below a threshold field, the particles rotated with the field. These rotating particles were therefore subject to a Magnus force that caused a measurable tilt of up to $1.2^{\circ}$ in their trajectories. The direction of the tilt agrees with rotation direction and with a simple model based on the Rubinov and Keller approximation for the Magnus force at low Reynolds numbers combined with linear viscous drag. The tilt angle increased with increasing rotation velocity and decreasing kinematic viscosity. The minimum field value for rotation increases with increasing rotation velocity and viscosity of the medium. Most of the particles no longer followed a magnetic field of $122 \mathrm{mT}$ in a medium with a kinematic viscosity of $2.310^{-6}$ $\mathrm{m}^{2} / s$ at a rotation velocity of $5 \mathrm{rps}$, and therefore no longer showed a tilt. The experiments clearly demonstrate that the Magnus force on particles with a diameter of tens of micrometers is measurable. The method allows for separation of particles based on the ratio between their magnetic moment and radius to the third power, which is of great interest for the analysis of magnetic microparticles such as catalyst particles or cells labeled with magnetic materials.

\section{Acknowledgements}

This work was supported by The Netherlands Centre for Multiscale Catalytic Energy Conversion (MCEC), an NWO Gravitation programme funded by the Ministry of Education, Culture and Science of the government of The Netherlands. 


\subsection{Appendix}

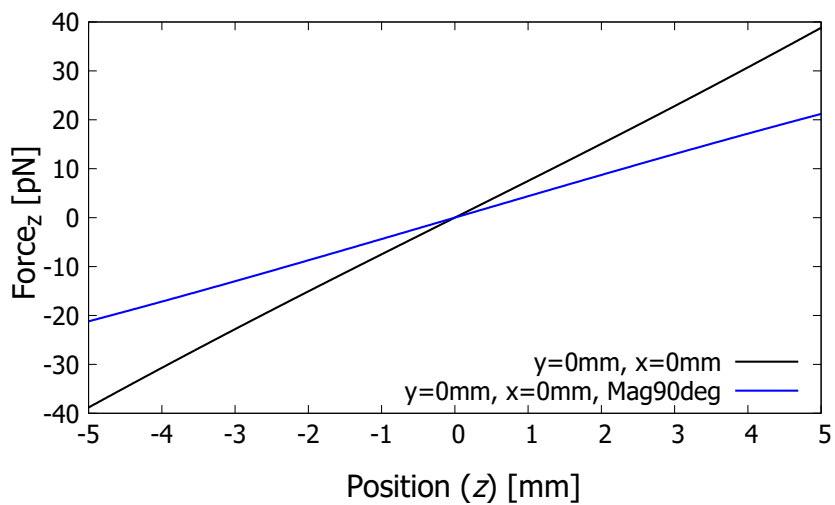

FIGURE 6.5 - Force on a paramagnetic sphere $(r=0.0169 \mathrm{~mm}, \mathrm{khi}=-0.016)$ between magnet plates as a function of its location.

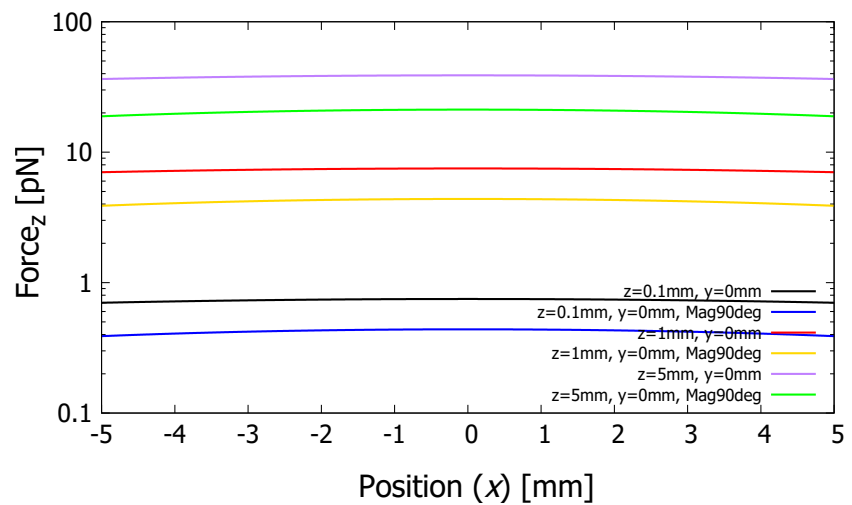

FIGURE 6.6 - Force on a paramagnetic sphere $(r=0.0169 m m, k h i=-0.016)$ between magnet plates as a function of its location. 
Chapter 6 - Trajectory Deflection of Spinning Magnetic Microparticles, the 84

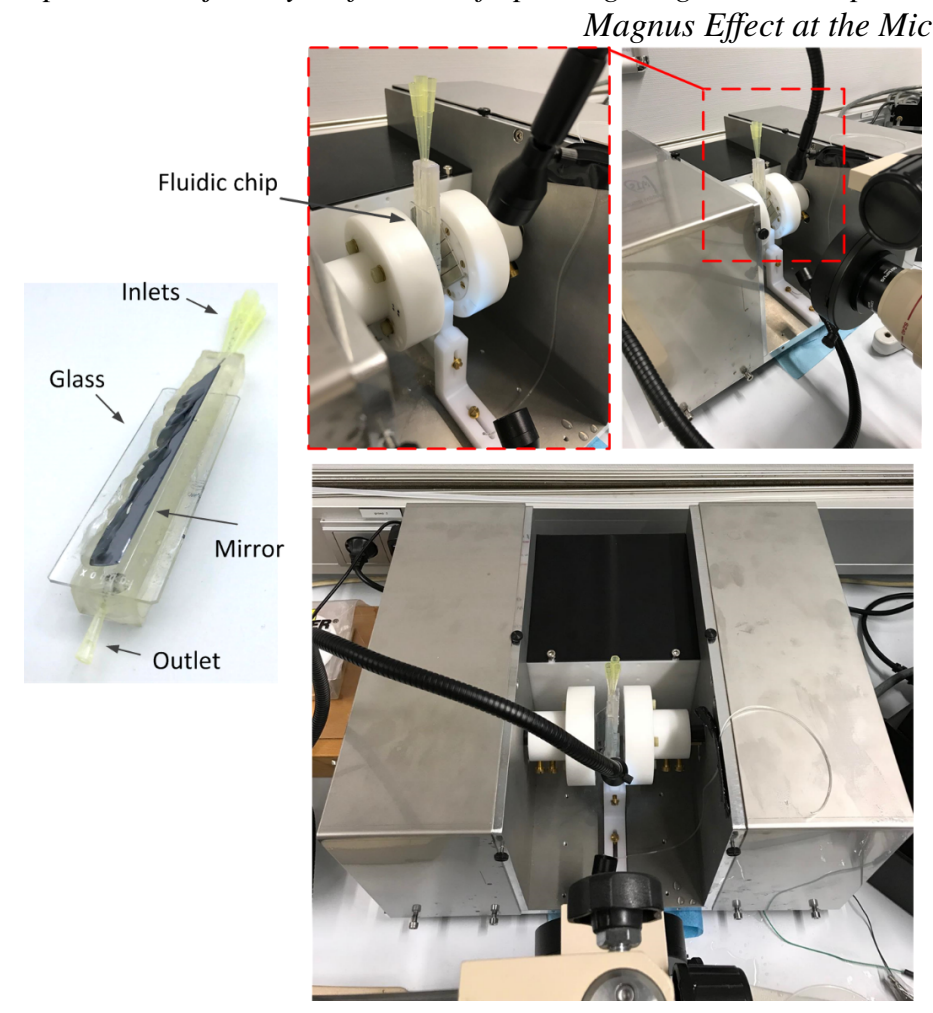

FIGURE 6.7 - Image of the fluidic system with a glass to seal the chip and observe the janus particles, a mirror to reflect the trajectories of the janus particles and 3 pipette tips to introduce the fluid and particles (left). Images of the setup where both magnets are placed at both sides of the fluidic chip (top). Top-view of the setup (bottom).

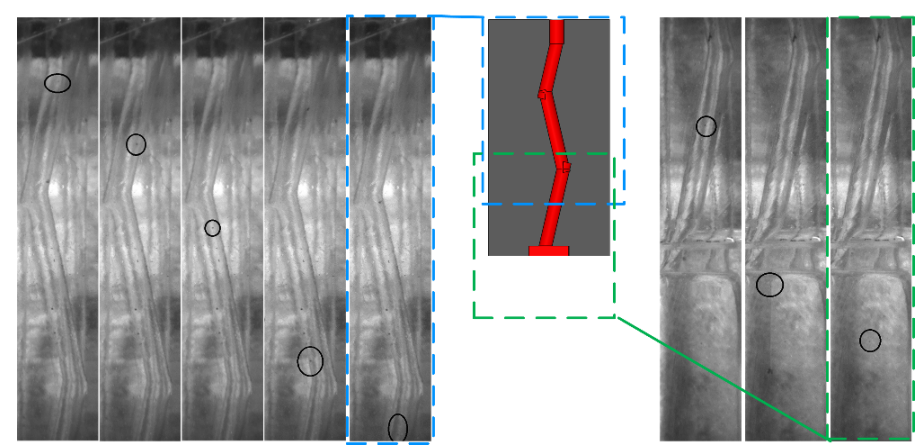

FIGURE 6.8 - Zig-zag inlet fabricated to introduce the janus particles at similar starting positions. Inside the black circle a particle rolling down can be followed until entering in the main channel. 

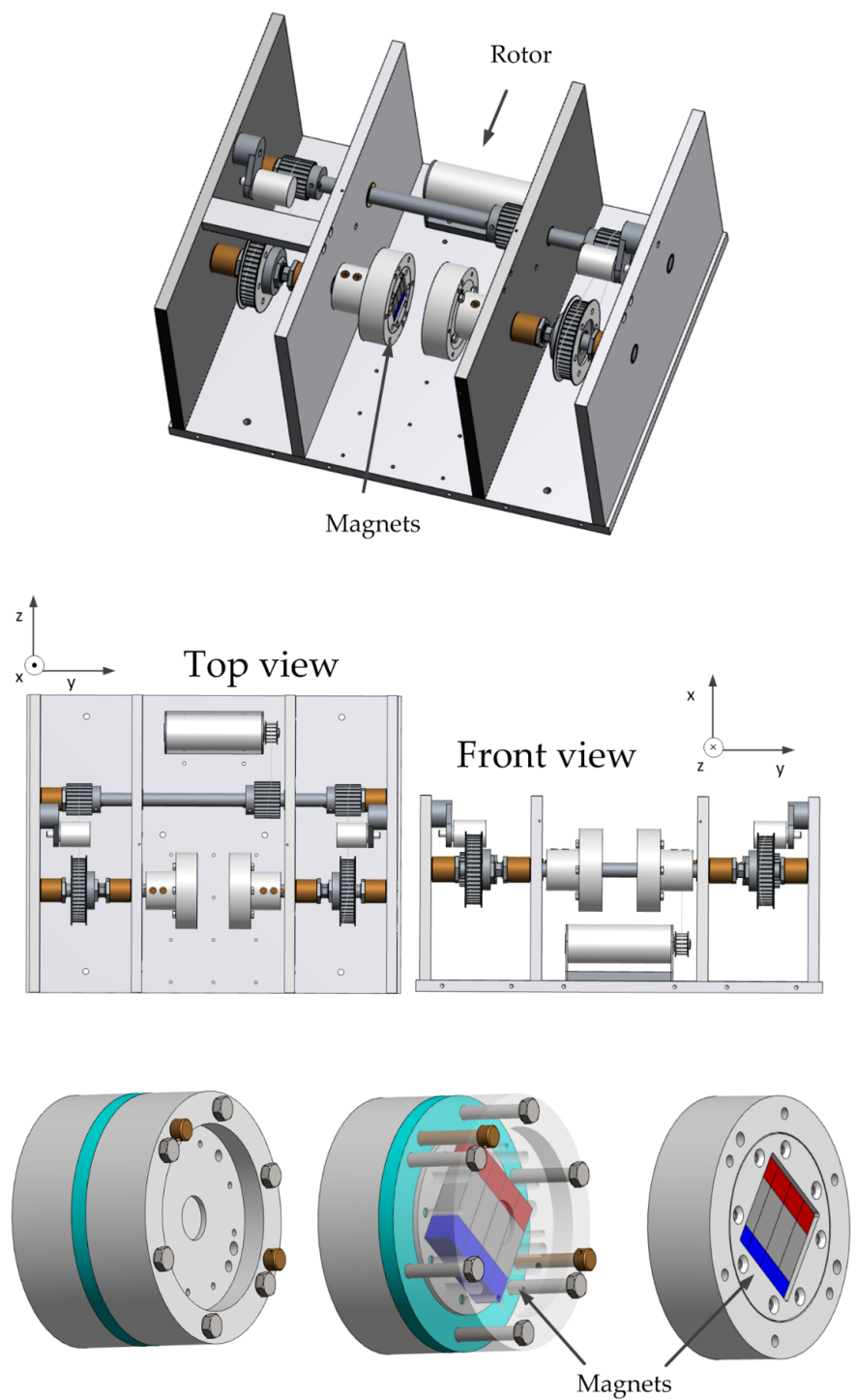

FIGURE 6.9 - Solidworks drawing of the rotating magnetic field setup where the electric motor rotates the magnets at the same rotational velocity and the mounting of magnets setup. 
Chapter 6 - Trajectory Deflection of Spinning Magnetic Microparticles, the 86 Magnus Effect at the Microscale

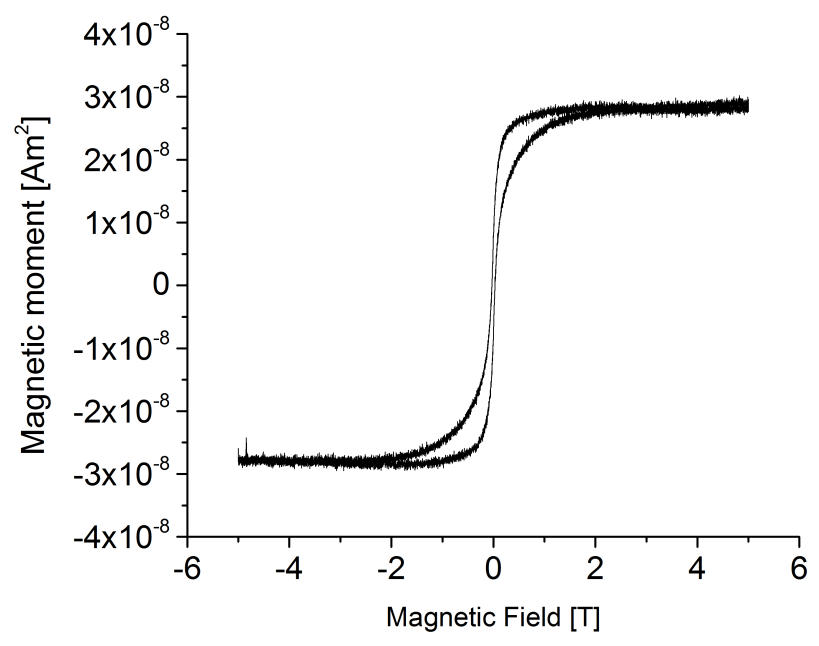

Figure 6.10 - Magnetic moment of 453 janus particles from -5 to $5 \mathrm{~T}$. 


\section{Chapter 7}

\section{Summary}

The content of this thesis ranges from magnetic material properties, to magnet designs, shimming methods to optimize their magnetic field homogeneity and a variety of applications of permanent magnet systems.

The research in chapter 2 aimed to compare a cuboid and a Pseudo-Halbach magnet configuration, in terms of their field strength and field homogeneity. For our optimized boundary conditions, we advise using the cuboid configuration for scientific use, where it is possible to preselect the permanent magnets and the external stray field is not a big issue. If preselecting the magnets is not an option, we recommend the Pseudo-Halbach configuration, which has a more robust homogeneity regarding variations in the magnetization and angle between the used magnets. Also the lower stray field makes this configuration easier to handle and therefore more favorable especially for industrial applications.

In chapter 3 and 4 we present three different ways to improve the field homogeneity of the Pseudo-Halbach. Using mechanical shimming, in our case two movable rings, brings two advantages.

First is a higher field, second a way to shim the field by sliding the rings closer together or further apart, depending of the aimed region of interest. This design is, caused by the amount of magnets and additional cage material to keep the magnets in place, more expensive than the earlier mentioned configurations but due to the easy handling an enjoyable magnet to work with.

We further improved the field with two planar shimming chips which uses two striplines and one conductive ring each, to generate a field which gets superimposed with the magnet field, resulting in a much better homogeneity.

The third method is a pilot study which aims for a better field-homogeneity by using a small amount of ferrofluid in a microfluidic chip. We have shown 
the possible usage of this method by alternating the volumes and the saturation magnetization of the fluid. With including more advanced fabrication techniques for the microfluidic chips, this method could be used in future for minimized magnet designs where the fabrication accuracy of permanent magnets is reached.

In chapter 5 we present a microfluidic Coriolis sensor, whose gain could be improved with either placing a magnet right on top of the chip, or placing a Halbach-like configuration around the channel.

Chapter 6 shows a new technique to sort magnetic microparticles by the Magnus force. Using two parallel magnets to spin the particles results in a drift, which deflection side depends on the spinning direction. With this technique, the particles could be sorted depending on their size and/or weight. 


\section{Samenvatting}

Dit proefschrift beschrijft een verscheidenheid aan onderwerpen, waaronder de magnetische eigenschappen van materialen, het ontwerpen van magneten, shimming-methoden om de homogeniteit van het magnetische veld te optimaliseren, en een verschillende toepassingen van permanente magneetsystemen.

In hoofdstuk 2 hebben we de magnetische eigenschappen van permanente magneten gemeten, maar konden geen opmerkelijke verschillen waarnemen tussen magneten van verschillende fabrikanten, materialen en coatings. We hebben een kubusvormige als ook een Pseudo-Halbach magneetconfiguratie met elkaar vergeleken, in termen van de sterkte en homogeniteit van het veld. We bevelen aan om voor wetenschappelijke doelienden de kubusvormige configuratie te gebruiken, waarbij de voorselectie van permanente magneten mogelijk is en het externe strooiveld niet problematisch is. Indien de magneten niet op voorhand kan worden geselecteerd, bevelen wij de Pseudo-Halbach-configuratie aan, omdat die volgens onze uitgevoerde Monte-Carlo simulaties een robustere homogeniteit heeft met betrekking tot variaties in de magnetisatie en de magnetisatiehoek. Daarnaast is deze configuratie door het lagere strooiveld makkelijker hanteerbaar en daardoor gunstiger, voor industriële toepassingen.

In hoofdstuk 3 en 4 presenteren we drie verschillende manieren om de homogeniteit van het veld ten gevolge van de Pseudo-Halbach configuratie te verbeteren. Het gebruik van mechanische shimming, in ons geval verplaatsbare ringen, brengt twee voordelen met zich mee.

Ten eerste is er een hoger veld, ten tweede is het een manier om het veld te shimmen door de ringen dichter of verder van elkaar te schuiven, op basis van de beoogde gevoeligheid. Dit ontwerp is duurder dan de eerder genoemde twee configuraties door het aantal magneten en extra materiaal voor de kooi die de magneten op hun plaats houdt, maar door de eenvoudige bediening prettig om mee te werken.

We hebben het veld verder verbeterd met twee vlakke shimming-chips die twee striplijnen gebruiken en elk één geleidende ring om een veld te genereren die wordt gesuperponeerd met het andere veld, resulterend in een veel betere homogeniteit. 
De derde methode is pilot studie die streeft naar een betere homogeniteit van het veld door een kleine hoeveelheid van ferrovloeistoffen te gebruiken in een microfluïdische chip. We hebben het mogelijke gebruik van deze methode laten zien door de volumes en de verzadigingsmagnetisatie van de vloeistof af te wisselen. Met behulp van geavanceerdere fabricagetechnieken voor microfluïdische chips, zou deze methode in de toekomst kunnen worden gebruikt voor geminimaliseerde magneet ontwerpen waarbij de fabricage nauwkeurigheid van permanente magneten wordt bereikt.

In hoofdstuk 5 beschrijven wij het ontwerp van een microfluïdische coriolis-sensor, waarvan de versterking kan worden verbeterd door een magneet te positioneren op de chip, of het plaatsen van een Halbach-achtige configuratie rondom het kanaal.

Hoofdstuk 6 toont een nieuwe techniek om magnetische microdeeltjes te sorteren door middel van het Magnuseffect. Door 2 parallelle magneten te gebruiken om de deeltjes te laten draaien, ontstaat een drift waarbij de afbruigrichting afhankelijk is van de draairichting. Door gebruik te maken van deze techniek, kunnden de deeltjes worden gesorteerd op basis van hun grootte en/of gewicht. 


\section{Zusammenfassung}

Der Inhalt dieser Arbeit reicht von der Untersuchung der Eigenschaften magnetischer Materialien über die Entwicklung von Magnetkonfigurationen, Methoden zur Verbesserung der Feldhomogenität und einer Vielzahl von Anwendungen von Dauermagnetsystemen.

Kapitel 2 befasst sich dem Vergleich zweier Magnetkonfigurationen (2 parallele Magnetquader und eine Pseudo-Halbach) bezüglich derer jeweiligen Magnetfeldstärke und Feldhomogenität. Unsere Untersuchungen ergaben, dass die Konfiguration die aus 2 Magnetquadern bestand, für den wissenschaftlichen Gebrauch besser geeignet ist, da hier eine Vorauswahl der verwendeten Permanentmagneten getroffen werden kann und Streufelder in der Regel keine große Rolle spielen. Sollte eine Vorauswahl der Magnete nicht möglich sein, beispielsweise für industrielle Anwendungen, empfehlen wir die Pseudo-Halbach Konfiguration. Die hier erreichte Homogenität ist im Vergleich schlechter, dafür aber vorhersehbar und robust bezüglich der Variation zwischen den Einzelmagneten. Die Ergebnisse basieren auf umfassenden Sensitivitätsanalysen und Monte-Carlo Simulationen.

In Kapitel 3 und 4 stellen wir drei Methoden vor, um die Feld-Homogenität der Pseudo-Halbach Konfiguration zu verbessern.

Die erste ist rein mechanisch: Die Verwendung von 2 speziell magnetisierten Ringe bringen 2 Vorteile. Einerseits wird die Feldstärke erhöht, andererseits kann die Feldhomogenität durch Einstellen der Ringposition verbessert werden. Diese Konfiguration ist durch die höhere Magnetanzahl etwas teurer und aufwändiger in der Montage, durch die einfache Handhabung ist sie für die Erforschung neuer Methoden zur Verbesserung der Feldhomogenität prädestiniert. In der zweiten Methode konnte die Homogenität durch zwei parallel angeordnete Chips im Innern der Konfiguration zusätzlich durch Überlagerung eines elektromagnetischen Feldes verbessert werden.

Kapitel 4 präsentiert eine gänzlich neue Methode: Die Verwendung von Ferrofluiden in Mikrofluidik-Chips. In dieser Pilotstudie wurde das Feldprofil erfolgreich durch Anpassung des Volumens bzw. der Sättigungsmagnetisierung der Ferrofluide verändert, um letztendlich die Feldhomogenität zu verbessern. 
Kapitel 5 und 6 zeigen mögliche mikrofluidische Anwendungen von Permanentmagnetsystemen.

In Kapitel 5 wird die Signaltstärke eines Coriolis-Sensors durch verschiedene Magnetkonfigurationen signifikant verbessert.

Kapitel 6 zeigt eine neuartige Methode, um magnetische Partikel zu sortieren. Die Partikel werden durch 2 parallele Magnete in Rotation versetzt und driften basierend auf dem Magnus-Effekte ab. Die Stärke der Abweichung ist abhängig von deren Gewicht und Größe. 


\section{Acknowledgments}

The work presented in this thesis has been done in a highly collaborative environment, including colleagues from KROHNE New Technologies BV, BRONKHORST Netherland BV, TU Delft (ME), KIST Europe Forschungsgesellschaft $\mathrm{mbH}$ and UTwente (MCS, IDS, BIOS Lab-on-a-chip).

I am deeply indebted to my supervisor Han who provided me with every bit of guidance and expertise during my whole Ph.D. project. You gave me freedom to branch out into new research areas, at the same time you contributed valuable feedback and advice. Thank you!

I owe a great debt of gratitude to Leon, who always appreciated my efforts, provided joyful encouragement and extraordinary support during the period of my master studies and this PhD project. You showed me the how amazing research can be, how tackle every problem, every challenge and every research question with cheerful enthusiasm.

I am also grateful to all colleagues from KROHNE New Technologies BV and TU Delft for fruitful discussions during our regular meetings, special thanks to Jankees, Lucas, Kofi and Eren. My sincere thanks to all user committee members for their constructive criticism and advice. I gratefully acknowledge the members of my Ph.D. committee for their time and valuable feedback on a pre-liminary version of this thesis. I extend thanks to all coauthors, especially Thomas and Miquel, for their important contributions in this doctoral project. Most of this work has been carried out in the MCS group at UTwente where I had the privilege to work in a truly international environment. I would like to thank my former and current colleagues for their input in brainstorm sessions, cakes, laughs and excellent technical help. I am also particularly grateful to all my flat-mates and friends for the pleasant conversations during lunch/coffee breaks and occasional gatherings. Finally but most importantly I would like to thank my family and Anna. Your unconditional love have been the biggest encouragement throughout this journey. 


\section{Bibliography}

Ahmed A, Hassan I, Mosa I M, Elsanadidy E, Sharafeldin M, Rusling J F, Ren S, jan 2019

"An ultra-shapeable, smart sensing platform based on a multimodal ferrofluid-infused surface"

Advanced Materials 31, p. 1807201, doi: 10.1002/adma.201807201

Alexiou C, Arnold W, Hulin P, Klein R, Schmidt A, Bergemann C, Parak F, 2001

"Magnetohydrodynamics"

doi: $10.22364 / \mathrm{mhd}$

Ambrisi A, Formisano A, Martone R, 2010

"Tolerance analysis of nmr magnets"

IEEE Transactions on Magnetics 46, pp. 2747-2750, doi: 10.1109/TMAG.2010.2043937

Amini H, Lee W, Carlo D D, 2014

"Inertial microfluidic physics"

Lab on a Chip 14, p. 2739, doi: 10.1039/c4lcoo128a

Anderson W A, mar 1961

"Electrical current shims for correcting magnetic fields"

Review of Scientific Instruments 32, pp. 241-25o, doi: 10.1063/1.1717338

Aspect, 2020.11.04

"Al-6o"

https://www.aspectimaging.com/industrial-solutions/ai-6o-nmr-lab-analyzer

Bagchi P, Balachandar S, aug 2002

"Effect of free rotation on the motion of a solid sphere in linear shear flow at moderate re" Physics of Fluids 14, pp. 2719-2737, doi: 10.1063/1.1487378

Barkla H M, Auchterlonie L J, jun 1971

"The magnus or robins effect on rotating spheres"

Journal of Fluid Mechanics 47, pp. 437-447, doi: 10.1017/soo22112071001150

Beck D, 2018

Magnus Force Acting Upon a Rotating Sphere Passing in an Incompressible Viscous Flow Ph.D. thesis, Charles University

Berg C H, 1993

Random walks in biology,

Princeton University Press

Blümich B, Blümler P, Eidmann G, Guthausen A, Haken R, Schmitz U, Saito K, Zimmer G, jun 1998 "The NMR-mouse: construction, excitation, and applications"

Magnetic Resonance Imaging 16, pp. 479-484, doi: 10.1016/so730-725x(98)ooo69-1 
BM Berkovsky V B, 1996

Magnetic fluids and applications handbook, New York ; Wallingford : Begell House

de Boer M, Tjerkstra R, Berenschot J, Jansen H, Burger G, Gardeniers J, Elwenspoek M, van den Berg A, mar 2000

"Micromachining of buried micro channels in silicon"

Journal of Microelectromechanical Systems 9, pp. 94-103, doi: 10.1109/84.825783

Carlson J B, sep 1975

"Lodestone compass: Chinese or olmec primacy?: Multidisciplinary analysis of an olmec hematite artifact from san lorenzo, veracruz, mexico"

Science 189, pp. 753-76o, doi: 10.1126/science.189.4205.753

Chen J, Xu C, 2007

"Design and analysis of the novel test tlibe magnet as a device for portable nuclear magnetic resonance"

IEEE Transactions on Magnetics 43, pp. 3555-3557, doi: 10.1109/TMAG.2007.901888

Chung Y C, Chen P W, Fu C M, Wu J M, may 2013

"Particles sorting in micro-channel system utilizing magnetic tweezers and optical tweezers"

Journal of Magnetism and Magnetic Materials 333, pp. 87-92, doi: 10.1016/j.jmmm.2012.12.041

Chung Y C, Wu C M, Lin S H, jun 2016

"Particles sorting in micro channel using designed micro electromagnets of magnetic field gradient"

Journal of Magnetism and Magnetic Materials 407, pp. 209-217, doi:

10.1016/j.jmmm.2016.01.075

Coey J, aug 2002

"Permanent magnet applications"

Journal of Magnetism and Magnetic Materials 248, pp. 441-456, doi: 10.1016/so3o48853(02)00335-9

Crozier S, Dodd S, Luescher K, Field J, Doddrell D M, mar 1995

"The design of biplanar, shielded, minimum energy, or minimum power pulsed bo coils" Magma: Magnetic Resonance Materials in Physics, Biology, and Medicine 3, pp. 49-55, doi: $10.1007 / \mathrm{bfo} 2426400$

Danieli E, Perlo J, Blümich B, Casanova F, may 2010 "Small magnets for portable NMR spectrometers" Angewandte Chemie International Edition 49, pp. 4133-4135, doi: 10.1002/anie.201000221

Delinchant B, Duret D, Estrabaut L, Gerbaud L, Huu H N, Peloux B D, Rakotoarison H, Verdiere F, Wurtz F, 2007

"An optimizer using the software component paradigm for the optimization of engineering systems"

COMPEL - The international journal for computation and mathematics in electrical and electronic engineering 26, pp. 368-379, doi: 10.1108/03321640710727728

Demas V, Prado P J, jan 2009

"Compact magnets for magnetic resonance"

Concepts in Magnetic Resonance Part A 34A, pp. 48-59, doi: 10.1002/cmr.a.20131

Denisov S I, Lyutyy T V, Reva V V, Yermolenko A S, mar 2018

"Temperature effects on drift of suspended single-domain particles induced by the magnus force" Physical Review E 97, doi: 10.1103/physreve.97.032608 
Enoksson P, Stemme G, Stemme E, 1997

"A silicon resonant sensor structure for coriolis mass-flow measurements"

Journal of microelectromechanical systems 6, pp. 119-125

Faraday M, 1821

"New electro-magnetic apparatus"

Quarterly Journal of Science

Forbes J C, aug 2015

"Curveballs in protoplanetary discs - the effect of the magnus force on planet formation" Monthly Notices of the Royal Astronomical Society 453, pp. 1779-1792, doi: 10.1093/mnras/stv1712

Gardeniers J, Bart J, Kolkman A, de Vries A J, Janssen J, van Bentum P, Ampt K, Wijmenga S, Kentgens A, jun 2009

"Microfluidic high-resolution NMR chip for biological fluids"

In: TRANSDUCERS 2009 - 2009 International Solid-State Sensors, Actuators and Microsystems

Conference, IEEE, doi: 10.1109/sensor.2009.5285775

Golay M J E, apr $195^{8}$

"Field homogenizing coils for nuclear spin resonance instrumentation"

Review of Scientific Instruments 29, pp. 313-315, doi: 10.1063/1.1716184

Gossett D R, Weaver W M, Mach A J, Hur S C, Tse H T K, Lee W, Amini H, Carlo D D, apr 2010 "Label-free cell separation and sorting in microfluidic systems"

Analytical and Bioanalytical Chemistry 397, pp. 3249-3267, doi: 10.1007/soo216-010-3721-9

Gou Y, Jia Y, Wang P, Sun C, jun 2018

"Progress of inertial microfluidics in principle and application"

Sensors 18, p. 1762, doi: 10.339o/s18061762

Gramme Z T, 1871

"Improvement in magneto-electric machines"

U.S. Patent US120057A, 1871

Groenesteijn J, Lammerink T, Wiegerink R, Haneveld J, Lötters J, 2012

"Optimization of a micro coriolis mass flow sensor using lorentz force actuation"

Sensors and Actuators A: Physical 186, pp. 48-53

Groenesteijn J, de Boer M, Lötters J, Wiegerink R, $2017 a$

"A versatile technology platform for microfluidic handling systems, part i: fabrication and functionalization"

Microfluidics and nanofluidics 21, p. 127

Groenesteijn J, de Boer M, Lötters J, Wiegerink R, $2017 b$

"A versatile technology platform for microfluidic handling systems, part ii: channel design and technology"

Microfluidics and nanofluidics 21, p. 127

Ha D, Paulsen J, Sun N, Song Y Q, Ham D, aug 2014

"Scalable NMR spectroscopy with semiconductor chips"

Proceedings of the National Academy of Sciences 111, pp. 11955-1196o, doi:

10.1073/pnas.1402015111

Haavisto M, Kankaanpaa H, Paju M, jan 2011

"Estimation of time-dependent polarization losses in sintered ndfeb permanent magnets"

IEEE Transactions on Magnetics 47, pp. 170-174, doi: 10.1109/tmag.2010.2089692 
Halbach K, jun 1979

"Strong rare earth cobalt quadrupoles"

IEEE Transactions on Nuclear Science 26, pp. 3882-3884, doi: 10.1109/tns.1979.4330638

Halbach K, 1980

"Design of permanent multipole magnets with oriented rare earth cobalt material"

Nuclear instruments and methods 169, pp. 1-10

Halbach K, 1985

"Permanent magnets for production and use of highenergy particle beams"

Proceedings of the Eighth International Workshop on Rare Earth Cobalt Permanent Magnets and their Applications p. 103

Haneveld J, Lammerink T, de Boer M, Sanders R, Mehendale A, Lötters J, Dijkstra M, Wiegerink R, 2010

"Modeling, design, fabrication and characterization of a micro coriolis mass flow sensor"

Journal of micromechanics and microengineering 20, p. 125001

Hashi K, Ohki S, Matsumoto S, Nishijima G, Goto A, Deguchi K, Yamada K, Noguchi T, Sakai S, Takahashi M, Yanagisawa Y, Iguchi S, Yamazaki T, Maeda H, Tanaka R, Nemoto T, Suematsu H, Miki T, Saito K, Shimizu T, jul 2015

"Achievement of $1020 \mathrm{MHz}$ NMR"

Journal of Magnetic Resonance 256, pp. 30-33, doi: 10.1016/j.jmr.2015.04.009

Hergt R, Andra W, Ambly C, Hilger I, Kaiser W, Richter U, Schmidt H G, 1998

"Physical limits of hyperthermia using magnetite fine particles"

IEEE Transactions on Magnetics 34, pp. 3745-3754, doi: 10.1109/20.718537

Hills B, Wright K, Gillies D, aug 2005

"A low-field, low-cost halbach magnet array for open-access NMR"

Journal of Magnetic Resonance 175, pp. 336-339, doi: 10.1016/j.jmr.2005.04.015

Horton J, Wright J, Herchenroeder J, 1996

"Fracture toughness of commercial magnets"

IEEE Transactions on Magnetics 32, pp. 4374-4376, doi: 10.1109/20.538873

Hua J, Yia H, Yina Q, Zhoua X, Lu R, Nia Z, 2017

"Design of a multilayer halbach permanent magnet for human finger nmr detection"

Applied Electromagnetics and Mechanics 54, pp. 315-327

Hugon C, D'Amico F, Aubert G, Sakellariou D, jul 2010

"Design of arbitrarily homogeneous permanent magnet systems for NMR and MRI: Theory and experimental developments of a simple portable magnet"

Journal of Magnetic Resonance 205, pp. 75-85, doi: 10.1016/j.jmr.2010.04.003

Huh D, Bahng J H, Ling Y, Wei H H, Kripfgans O D, Fowlkes J B, Grotberg J B, Takayama S, feb 2007

"Gravity-driven microfluidic particle sorting device with hydrodynamic separation amplification" Analytical Chemistry 79, pp. 1369-1376, doi: 10.1021/aco61542n

IV C W S, Reyes C D, López G P, 2015

"Microfluidic cell sorting: a review of the advances in the separation of cells from debulking to rare cell isolation"

Lab on a Chip 15, pp. 1230-1249, doi: 10.1039/c4lco1246a

JC Edwards P G, 2010

Process Analytical Technology: Spectroscopic Tools and Implementation Strategies for the Chemical and Pharmaceutical Industries,

Blackwell Publishing

ISBN 978-0-470-72207-7 
Kalfe A, Telfah A, Lambert J, Hergenröder R, jul 2015

"Looking into living cell systems: Planar waveguide microfluidic NMR detector for in vitro metabolomics of tumor spheroids"

Analytical Chemistry 87, pp. 7402-7410, doi: 10.1021/acs.analchem.5bo16o3

Kensrud J R, 2010

Determining aerodynamic properties of sports balls in situ

Master's thesis, Washington State University

Kong T F, E H S, Sugiarto H S, Liew H F, Wang X, Lew W S, Nguyen N T, Chen Y, nov 2010

"An efficient microfluidic sorter: implementation of double meandering micro striplines for magnetic particles switching"

Microfluidics and Nanofluidics 1o, pp. 1069-1078, doi: 10.1007/s10404-010-0735-8

Kray T, Franke J, Frank W, nov 2012

"Magnus effect on a rotating sphere at high reynolds numbers"

Journal of Wind Engineering and Industrial Aerodynamics 110, pp. 1-9, doi:

10.1016/j.jweia.2012.07.005

Kreyenschulte D, Paciok E, Regestein L, Blümich B, Büchs J, may 2015

"Online monitoring of fermentation processes via non-invasive low-field NMR"

Biotechnology and Bioengineering 112, pp. 1810-1821, doi: 10.1002/bit.25599

Lee C J, Lee C I, Jang G H, jul 2011

"Source and reduction of uneven magnetization of the permanent magnet of a HDD spindle motor"

IEEE Transactions on Magnetics 47, pp. 1929-1932, doi: 10.1109/tmag.2011.2123877

Lee H, Sun E, Ham D, Weissleder R, jul 2008

"Chip-NMR biosensor for detection and molecular analysis of cells"

Nature Medicine 14, pp. 869-874, doi: 10.1038/nm.1711

Levitt M, 2008

Spin Dynamics: Basics of Nuclear Magnetic Resonance,

Wiley, 2 edition

ISBN 13-978-0470511176

Li P, Mao Z, Peng Z, Zhou L, Chen Y, Huang P H, Truica C I, Drabick J J, El-Deiry W S, Dao M, Suresh S, Huang T J, apr 2015

"Acoustic separation of circulating tumor cells"

Proceedings of the National Academy of Sciences 112, pp. 4970-4975, doi:

10.1073/pnas.1504484112

Li Y, Evans H, Harris I, Jones I, 2003

"The oxidation of ndfeb magnets"

Oxidation of Metals 59, pp. 167-182, doi: 10.1023/a:1023078218047

Li Y F, Zhu M G, Li W, Zhou D, Lu F, Chen L, Wu J Y, Qi Y, Du A, sep 2013

"The impact induced demagnetization mechanism in ndfeb permanent magnets"

Chinese Physics Letters 3o, p. 097501, doi: 10.1088/0256-307x/30/9/097501

Lindsay R B, oct 1940

"William gilbert and magnetism in 1600"

American Journal of Physics 8, pp. 271-282, doi: 10.1119/1.1991585

Liu C, Stakenborg T, Peeters S, Lagae L, may 2009

"Cell manipulation with magnetic particles toward microfluidic cytometry"

Journal of Applied Physics 105, p. 102014, doi: 10.1063/1.3116091 
Liu W, Zu D, Tang X, Guo H, jul 2007

"Target-field method for MRI biplanar gradient coil design"

Journal of Physics D: Applied Physics 4o, pp. 4418-4424, doi: 10.1088/0022-3727/40/15/005

Livingston J D, feb 1990

"The history of permanent-magnet materials"

JOM 42, pp. 30-34, doi: $10.1007 /$ bfo3220870

Lu X, Xuan X, jun 2015

"Continuous microfluidic particle separation via elasto-inertial pinched flow fractionation"

Analytical Chemistry 87, pp. 6389-6396, doi: 10.1021/acs.analchem.5bo1432

Magnus G, 1853

"über die abweichung der geschosse, und eine auffallende erscheinung bei rotierenden körpern"

Poggendorfs Ann. der Phys. und Chemie $\mathbf{8 8}$

Marble A E, Mastikhin I V, Colpitts B G, Balcom B J, may 2005

"An analytical methodology for magnetic field control in unilateral NMR"

Journal of Magnetic Resonance 174, pp. 78-87, doi: 10.1016/j.jmr.2005.01.009

Martens M A, Petropoulos L S, Brown R W, Andrews J H, Morich M A, Patrick J L, nov 1991

"Insertable biplanar gradient coils for magnetic resonance imaging"

Review of Scientific Instruments 62, pp. 2639-2645, doi: 10.1063/1.1142245

McDonnell E E, Han S, Hilty C, Pierce K L, Pines A, 2005

"Nmr analysis on microfluidic devices by remote detection"

Anal. Chem. 77, pp. 8109-8114

van Meerten S G J, van Bentum P J M, Kentgens A P M, aug 2018

"Shim-on-chip design for microfluidic NMR detectors"

Analytical Chemistry 9o, pp. 10134-10138, doi: 10.1021/acs.analchem.8bo2284

Meethan W, Thomas I, Thomas C, 2014

"Calculation of the sensitive region of a u-shaped permanent magnet for a single-sided NMR spectrometer"

Chiang Mai University Journal of Natural Sciences 13, doi: 10.12982/cmujns.2014.0021

Meribout M, Sonowan S, jan 2019

"Optimal halbach magnet array design for portable NMR targeting multiphase flow metering applications"

IEEE Transactions on Magnetics 55, pp. 1-7, doi: 10.1109/tmag.2018.2877603

Michell J, $175^{1}$

A Treatise of Artificial Magnets : In Which Is Shown An Easy And Expeditious Method Of Making Them, Superior To The Best Natural Ones,

Kessinger Legacy Reprints, Kessinger Publishing, Whitefish MT, USA

ISBN 9781166426088

Mirowski E, Moreland J, Zhang A, Russek S E, Donahue M J, jun 2005

"Manipulation and sorting of magnetic particles by a magnetic force microscope on a microfluidic magnetic trap platform"

Applied Physics Letters 86, p. 243901, doi: 10.1063/1.1947368

Mitchell J, Gladden L, Chandrasekera T, Fordham E, jan 2014

"Low-field permanent magnets for industrial process and quality control"

Progress in Nuclear Magnetic Resonance Spectroscopy 76, pp. 1-6o, doi

10.1016/j.pnmrs.2013.09.001 
Moerland C P, van IJzendoorn L J, Prins M W J, 2019

"Rotating magnetic particles for lab-on-chip applications - a comprehensive review"

Lab on a Chip 19, pp. 919-933, doi: 10.1039/c81c01323c

Moresi G, Magin R, 2003

"Miniature permanent magnet for table-top NMR"

Concepts in Magnetic Resonance 19B, pp. 35-43, doi: 10.1002/cmr.b.10082

Mozzhukhin G, Kupriyanova G, Mamadazizov S, Maraşlı A, Rameev B, sep 2018 "Low-field 14n nuclear magnetic resonance for detection of dangerous liquids" Chemical Physics 513, pp. 129-134, doi: 10.1016/j.chemphys.2018.07.032

Nanalysis, 2020.11.04

"Nmready"

www.nanalysis.com

Oersted H, 1820

"Experiments on the effect of a current of electricity on the magnetic needle" Annals of Philosophy

Oesterlé B, Dinh T B, jun 1998

"Experiments on the lift of a spinning sphere in a range of intermediate reynolds numbers" Experiments in Fluids 25, pp. 16-22, doi: 10.1007/s003480050203

Papell S, 1964

"Low viscosity magnetic fluid obtained by the colloidal suspension of magnetic particles"

US Patent 3215 572, 1964

PicoSpin8o, 2020.11.04

"Thermo scientific"

www.thermoscientific.com/en/products/nuclear-magnetic-resonance-nmr.html

Pinho M, Génevaux J, Dauchez N, Brouard B, Collas P, Mézière H, apr 2014

"Damping induced by ferrofluid seals in ironless loudspeaker"

Journal of Magnetism and Magnetic Materials 356, pp. 125-130, doi:

10.1016/j.jmmm.2013.12.047

Pulsar, 2020.11.04

"Oxford instruments"

www.oxford-instruments.com/products/spectrometers/nuclear-magnetic-resonance-nmr/pulsar

Raich H, Blümler P, oct 2004

"Design and construction of a dipolar halbach array with a homogeneous field from identical bar magnets: NMR mandhalas"

Concepts in Magnetic Resonance Part B: Magnetic Resonance Engineering 23B, pp. 16-25, doi: 10.1002/cmr.b.20018

Rem P C, Fraunholcz N, Schokker E A, dec 2002

"MAGNUS SEPARATION"

Separation Science and Technology 37, pp. 3647-366o, doi: 10.1081/ss-120014824

Roméo F, Hoult D I, mar 1984

"Magnet field profiling: Analysis and correcting coil design"

Magnetic Resonance in Medicine 1, pp. 44-65, doi: 10.1002/mrm.1910010107

Royce E B, oct 1966

"Anomalous shock-induced demagnetization of nickel ferrite"

Journal of Applied Physics 37, pp. 4066-4070, doi: 10.1063/1.1707977 
Rubinow S I, Keller J B, 1961

"The transverse force on a spinning sphere moving in a viscous fluid"

Fluid Mech. 11, p. 447 â€“"459

Saffman P G, jun 1965

"The lift on a small sphere in a slow shear flow"

Journal of Fluid Mechanics 22, pp. 385-400, doi: 10.1017/s0022112065000824

Sahebjavaher R S, Walus K, Stoeber B, feb 2010

"Permanent magnet desktop magnetic resonance imaging system with microfabricated multiturn gradient coils for microflow imaging in capillary tubes"

Review of Scientific Instruments 81, p. 023706, doi: 10.1063/1.3280171

Sajeesh P, Sen A K, nov 2013

"Particle separation and sorting in microfluidic devices: a review"

Microfluidics and Nanofluidics 17, pp. 1-52, doi: 10.1007/s10404-013-1291-9

Sakellariou D, Hugon C, Guiga A, Aubert G, Cazaux S, Hardy P, sep 2010

"Permanent magnet assembly producing a strong tilted homogeneous magnetic field: towards magic angle field spinning NMR and MRI"

Magnetic Resonance in Chemistry 48, pp. 903-908, doi: 10.1002/mrc.2683

Schut T, Klein Y, Wiegerink R, Lotters J, mar 2020

"Magnetic field strength improvement for lorentz actuation of a $\mu$-coriolis mass flow sensor"

Microelectronic Engineering 224, p. 111236, doi: 10.1016/j.mee.2020.111236

Seifert J, nov 2012

"A review of the magnus effect in aeronautics"

Progress in Aerospace Sciences 55, pp. 17-45, doi: 10.1016/j.paerosci.2012.07.001

Solsona M, Nieuwelink A E, Meirer F, Abelmann L, Odijk M, Olthuis W, Weckhuysen B M, van den Berg A, jul 2018

"Magnetophoretic sorting of single catalyst particles"

Angewandte Chemie International Edition 57, pp. 10589-10594, doi: 10.1002/anie.201804942

Solsona M, Vollenbroek J C, Tregouet C B M, Nieuwelink A E, Olthuis W, van den Berg A, Weckhuysen B M, Odijk M, 2019

"Microfluidics and catalyst particles"

Lab on a Chip 19, pp. 3575-36o1, doi: 10.1039/c9lcoo318e

Solsona M, Keizer H, de Boer H L, Klein Y P, Olthuis W, Abelmann L, van den Berg A, may 2020 "Trajectory deflection of spinning magnetic microparticles: The magnus effect at the microscale" Journal of Applied Physics 127, p. 194702, doi: 10.1063/1.5145064

Soltner H, Blümler P, jul 2010

"Dipolar halbach magnet stacks made from identically shaped permanent magnets for magnetic resonance"

Concepts in Magnetic Resonance Part A 36A, pp. 211-222, doi: 10.1002/cmr.a.20165

Sørensen M K, Vinding M S, Bakharev O N, Nesgaard T, Jensen O, Nielsen N C, jul 2014

"NMR sensor for onboard ship detection of catalytic fines in marine fuel oils"

Analytical Chemistry 86, pp. 7205-7208, doi: 10.1021/ac5014496

Sørensen M K, Jensen O, Bakharev O N, Nyord T, Nielsen N C, jun 2015

"NPK NMR sensor: Online monitoring of nitrogen, phosphorus, and potassium in animal slurry" Analytical Chemistry 87, pp. 6446-645o, doi: 10.1021/acs.analchem.5bo1924

Sparks D, Smith R, Cripe J, Schneider R, Najafi N, 2003

"A portable mems coriolis mass flow sensor"

In: IEEE SENSORS, pp. 337-339, 
Spinsolve, 2020.11.04

"Magritek"

www.magritek.com

Sun N, Liu Y, Qin L, Lee H, Weissleder R, Ham D, 2013

"Small NMR biomolecular sensors"

Solid-State Electronics 84, pp. 13 - 21, doi: https://doi.org/10.1016/j.sse.2013.02.005

Tamada D, Kose K, Haishi T, apr 2012

"A new planar single-channel shim coil using multiple circular currents for magnetic resonance imaging"

Applied Physics Express 5, p. 056701, doi: 10.1143/apex.5.056701

Tayler M C, Sakellariou D, apr 2017

"Low-cost, pseudo-halbach dipole magnets for NMR"

Journal of Magnetic Resonance 277, pp. 143-148, doi: 10.1016/j.jmr.2017.03.001

Tsuji Y, Morikawa Y, Mizuno O, dec 1985

"Experimental measurement of the magnus force on a rotating sphere at low reynolds numbers"

Journal of Fluids Engineering 107, pp. 484-488, doi: 10.1115/1.3242517

Vogel M W, Giorni A, Vegh V, Pellicer-Guridi R, Reutens D C, jun 2016

"Rotatable small permanent magnet array for ultra-low field nuclear magnetic resonance instrumentation: A concept study"

PLOS ONE 11, p. e0157040, doi: 10.1371/journal.pone.0157040

Volder M D, Reynaerts D, jun 2009

"Development of a hybrid ferrofluid seal technology for miniature pneumatic and hydraulic actuators"

Sensors and Actuators A: Physical 152, pp. 234-240, doi: 10.1016/j.sna.2009.04.010

Whitesides G M, jul 2006

"The origins and the future of microfluidics"

Nature 442, pp. 368-373, doi: 10.1038/nature05058

Windt C W, Soltner H, van Dusschoten D, BlÂ¹/4mler P, jan 2011

"A portable halbach magnet that can be opened and closed without force: The NMR-CUFF"

Journal of Magnetic Resonance 208, pp. 27-33, doi: 10.1016/j.jmr.2010.09.020

Yamada M, Seki M, feb 2006

"Microfluidic particle sorter employing flow splitting and recombining"

Analytical Chemistry 78, pp. 1357-1362, doi: 10.1021/ac0520083

You X F, Hu L L, Yang W H, Wang Z, Wang H X, jun 2010

"Biplanar shim coil design for $1.5 \mathrm{t}$ permanent magnet of in vivo animal MRI"

IEEE Transactions on Applied Superconductivity 2o, pp. 1045-1049, doi:

10.1109/tasc.2010.2041537

Yu W, Lin H, Wang Y, He X, Chen N, Sun K, Lo D, Cheng B, Yeung C, Tan J, Carlo D D, Emaminejad S, feb 2020

"A ferrobotic system for automated microfluidic logistics"

Science Robotics 5, p. eaba4411, doi: 10.1126/scirobotics.aba4411

Zalesskiy S S, Danieli E, Blümich B, Ananikov V P, apr 2014

"Miniaturization of NMR systems: Desktop spectrometers, microcoil spectroscopy, and "NMR on a chip" for chemistry, biochemistry, and industry"

Chemical Reviews 114, pp. 5641-5694, doi: 10.1021/cr400063g 
Zeng J, Deng Y, Vedantam P, Tzeng T R, Xuan X, nov 2013

"Magnetic separation of particles and cells in ferrofluid flow through a straight microchannel using two offset magnets"

Journal of Magnetism and Magnetic Materials 346, pp. 118-123, doi:

10.1016/j.jmmm.2013.07.021

Zhang J, Yan S, Yuan D, Alici G, Nguyen N T, Warkiani M E, Li W, 2016

"Fundamentals and applications of inertial microfluidics: a review"

Lab on a Chip 16, pp. 10-34, doi: 10.1039/c5lco1159k

Zhu T, Cheng R, Lee S A, Rajaraman E, Eiteman M A, Querec T D, Unger E R, Mao L, jun 2012 "Continuous-flow ferrohydrodynamic sorting of particles and cells in microfluidic devices" Microfluidics and Nanofluidics 13, pp. 645-654, doi: 10.1007/s10404-012-1004-9

Zhu Z, Howe D, 2001

"Halbach permanent magnet machines and applications: a review"

IEE Proceedings - Electric Power Applications 148, p. 299, doi: 10.1049/ip-epa:20010479 
ISBN:978-903-65-5102- 1 doi:10.3990/1.9789036551021 